Aus der Klinik für Kardiologie und Pneumologie

(Prof. Dr. med. G. Hasenfuß)

der Medizinischen Fakultät der Universität Göttingen

\title{
Strukturelle und funktionale Veränderungen der atrialen Kalzium-Freisetzungseinheit im Herzinsuffizienzmodell durch Junctophilin-2-Knockdown
}

\author{
INAUGURAL-DISSERTATION \\ zur Erlangung des Doktorgrades \\ der Medizinischen Fakultät der \\ Georg-August-Universität zu Göttingen
}

vorgelegt von

Benjamin Eikenbusch

aus

Berlin 
Dekan:

\section{Betreuungsausschuss}

Betreuer:

Ko-Betreuerin:

\section{Prüfungskommission}

Referent

Ko-Referentin:

Drittreferent:
Prof. Dr. med. Wolfgang Brück

Prof. Dr. med. Stephan E. Lehnart

Prof. Dr. med. Dörthe M. Katschinski
Prof. Dr. med. Stephan E. Lehnart

Prof. Dr. med. Dörthe M. Katschinski

Prof. Dr. mult. Thomas Meyer

Datum der mündlichen Prüfung: 18.03.2021 
Hiermit erkläre ich, die Dissertation mit dem Titel "Strukturelle und funktionale Veränderungen der atrialen Kalzium-Freisetzungseinheit im Herzinsuffizienzmodell durch Junctophilin-2-Knockdown" eigenständig angefertigt und keine anderen als die von mir angegebenen Quellen und Hilfsmittel verwendet zu haben.

Göttingen, den 27.01.2020

(Benjamin Eikenbusch) 
Die Daten, auf denen die vorliegende Arbeit basiert, wurden teilweise publiziert:

Brandenburg S, Pawlowitz J, Eikenbusch B, Peper J, Kohl T, Mitronova GY, Sossalla S, Hasenfuss G, Wehrens XH, Kohl P (2019): Junctophilin-2 expression rescues atrial dysfunction through polyadic junctional membrane complex biogenesis. JCI Insight $\underline{4}$ 


\section{Inhaltsverzeichnis}

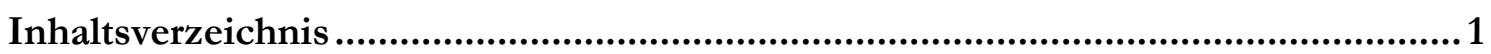

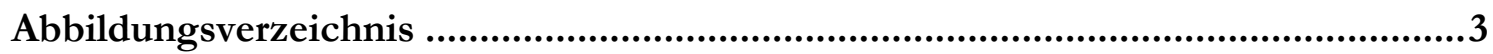

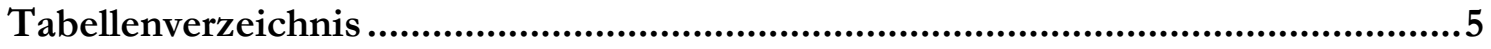

Abkürzungsverzeichnis ....................................................................................6

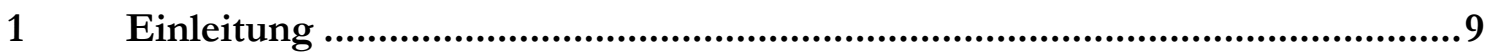

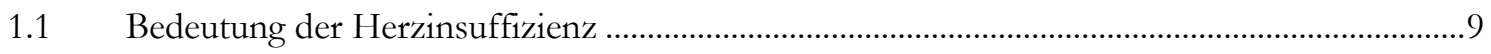

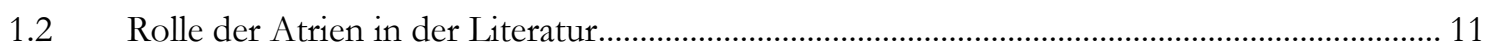

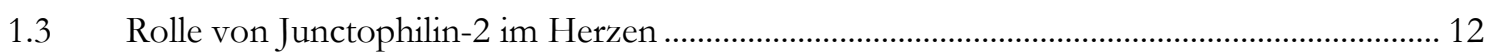

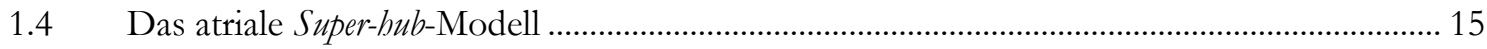

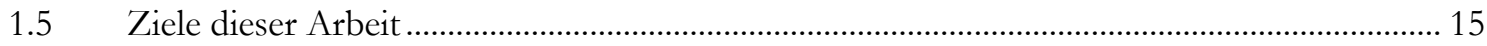

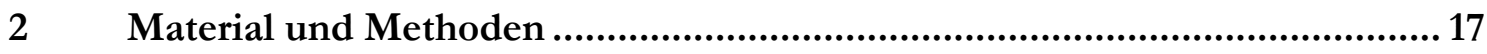

2.1 Transgene Mauslinien und Krankheitsmodelle .......................................................................... 17

2.2 Proteinbiochemische Analyse mittels Western-Blot ............................................................... 19

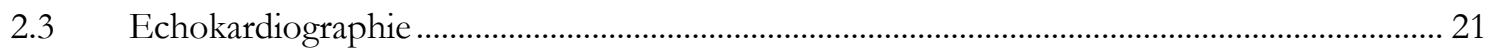

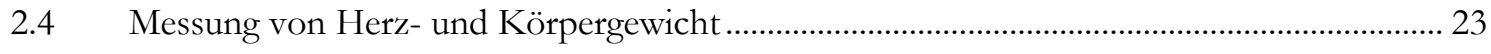

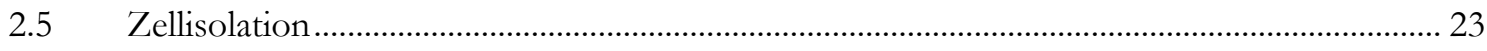

2.6 Konfokalmikroskopische Aufnahmen lebender Kardiomyozyten.......................................... 24

2.7 Analyse der konfokalen Aufnahmen lebender Kardiomyozyten.............................................. 25

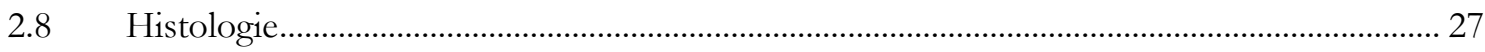

2.9 Konfokalmikroskopische und STED-Aufnahmen fixierter Kardiomyozyten mittels Immunfluoreszenzfärbung …………………………………………………………….... 28

2.10 Analyse von JPH2- und RyR2-Clustern anhand von STED-Aufnahmen fixierter

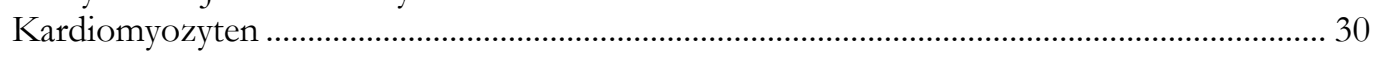

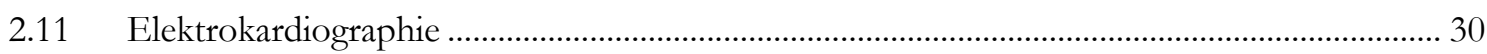

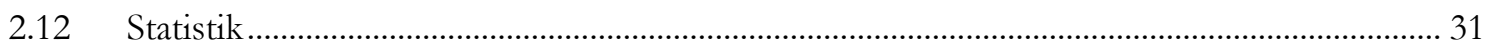

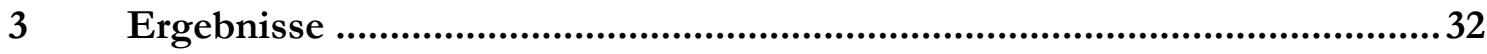

3.1 Proteinbiochemischer Nachweis der Expression von Junctophilin-2 im Atrium durch Western-Blot..................................................................................................................... 32

3.2 Reduzierte linkskardiale Kontraktilität ohne Hypertrophiezeichen nach Junctophilin-2-

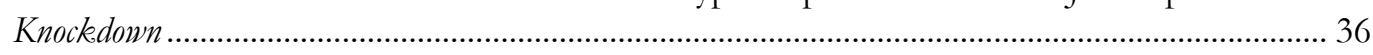

3.3 Erhöhtes Herzgewicht nach Junctophilin-2-Knockdown ……………………………………...... 38

3.4 Konfokalmikroskopische Aufnahmen lebender Kardiomyozyten........................................... 39

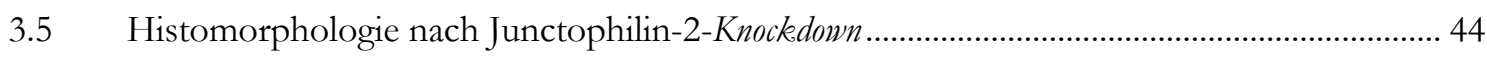

3.6 Konfokalmikroskopische und STED-Aufnahmen fixierter Kardiomyozyten mittels Immunfluoreszenz färbungen ................................................................................................... 47 
3.7 Erhöhte Herzfrequenz und verzögerte atrioventrikuläre Erregungsleitung nach Junctophilin-2-Knockdown

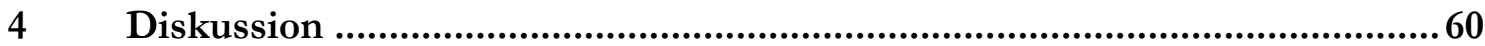

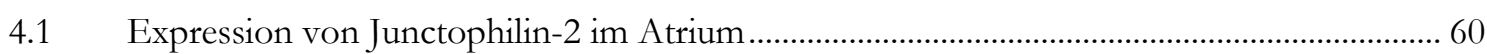

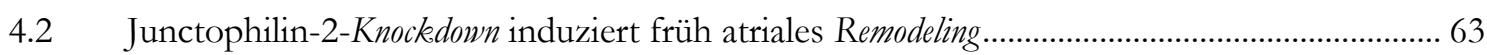

4.3 Differenzielle Verteilung von Junctophilin-2 in atrialen Myozyten...................................... 67

4.4 Verzögerung der atrialen Erregungsausbreitung im EKG ................................................ 67

$5 \quad$ Zusammenfassung und Ausblick ..................................................69

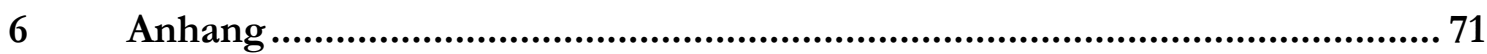

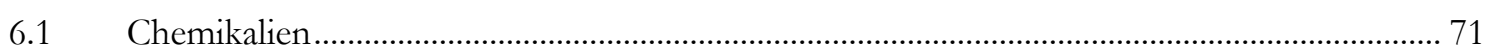

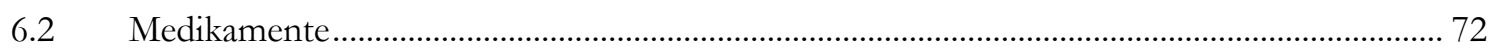

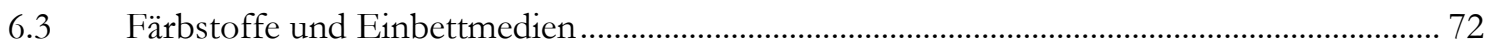

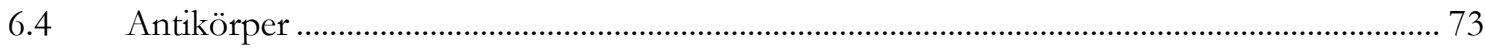

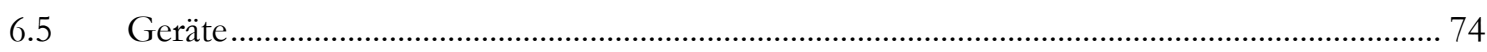

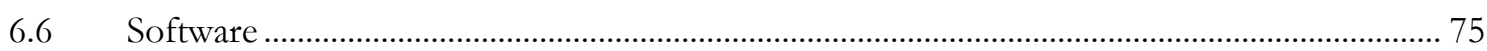

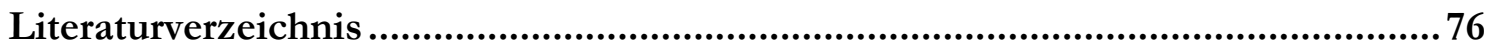




\section{Abbildungsverzeichnis}

Abbildung 1: Ablauf des Behandlungsprotokolls 2p40mg.

Abbildung 2: Nachweis der Proteinexpression von Junctophilin-2 im Ventrikel und Atrium muriner Wildtyp-Herzen.

Abbildung 3: Junctophilin-2-Knockdown ohne Mortalität nach Tamoxifen-Behandlungsschema 2p40mg.

Abbildung 4: Junctophilin-2-Knockdown führt zu atrialen Expressionsveränderungen kardial bedeutsamer Proteine.

Abbildung 5: Junctophilin-2-Knockdown induziert Verschlechterung funktioneller

Herzparameter 38

Abbildung 6: Herzgewicht-Zunahme in der Junctophilin-2-Knockdown-Gruppe.

Abbildung 7: Junctophilin-2-Knockdown führt nicht zu Hypertrophie atrialer Kardiomyozyten.

Abbildung 8: Verschiebung der Häufigkeiten der Tubulusausrichtungen nach Junctophilin-2-

Knockdown zugunsten axialer Tubuli.

Abbildung 9: Proliferation des TAT-Netzwerks und der axialen Tubuli nach Junctophilin-2-

Knockdown

Abbildung 10: Hämatoxylin-Eosin-Färbung schließt Hypertrophie nach Junctophilin-2-

Knockdown aus.

Abbildung 11: Sirius-Red/Fast-Green-Färbung zeigt keine atriale Fibrose nach Junctophilin-2-

Knockdown 46

Abbildung 12: Vergleich der Größe, Abundanz und Verteilung ventrikulärer und atrialer Junctophilin-2-Cluster anhand von STED-Aufnahmen (muriner Wildtyp).

Abbildung 13: Konfokale Aufnahmen der RyR2-pS2808/RyR2/JPH2-Färbung illustrieren sowohl die Kolokalisation großer Junctophilin-2-Cluster mit Clustern des hochphosphorylierten Ryanodin-Rezeptors Typ 2 als auch deren ähnliches Verteilungsmuster innerhalb der Zelle.

Abbildung 14: Konfokalmikroskopisch nachweisliche Kopplung großer Junctophilin-2-Cluster an axiale Tubuli in humanen atrialen Kardiomyozyten.

Abbildung 15: Konfokale und STED-Aufnahmen der JPH2/RyR2-Färbung illustrieren den Knockdown von Junctophilin-2 neben einem gestörten Cluster-Verhalten der Typ-2Ryanodin-Rezeptoren.

Abbildung 16: Konfokale und STED-Aufnahmen der JPH2/Cav3-Färbung zeigen eine verminderte Größe junktionaler Junctophilin-2-Cluster entlang axialer Tubuli.

Abbildung 17: Konfokale Aufnahmen der RyR2-pS2808/RyR2-Färbung zeigen die differenzielle Verteilung niedrigphosphorylierter und hochphosphorylierter Typ-2Ryanodinrezeptoren in atrialen Myozyten. 
Abbildung 18: Konfokale Aufnahmen der RyR2-pS2808/Cav3-Färbung belegen die junktionale Eigenschaft hochphosphorylierter Typ-2-Ryanodin-Rezeptoren an axialen Tubuli.

Abbildung 19: Konfokale und STED-Aufnahmen der Cav1.2/Cav3-Färbung zeigen die Lokalisation spannungsabhängiger L-Typ-Kalziumkanäle an Tubulusmembranstrukturen und Auswirkungen des Junctophilin-2-Knockdowns 56

Abbildung 20: Verzögerte atrioventrikuläre Erregungsleitung bei erhöhter Herzfrequenz nach Junctophilin-2-Knockdown 58 


\section{Tabellenverzeichnis}

Tabelle 1: Lösungen, Puffer und Gele für Western-Blots. .................................................................... 20

Tabelle 2: Morphologische Parameter der Echokardiographie............................................................. 22

Tabelle 3: Funktionelle Parameter der Echokardiographie. .............................................................. 22

Tabelle 4: Pufferlösungen für die Kardiomyozytenisolation............................................................ 24

Tabelle 5: Junctophilin-2-Knockdown im Atrium. .............................................................................. 33

Tabelle 6: Werte der atrialen Proteinexpression nach Junctophilin-2-Knockdown .............................. 35

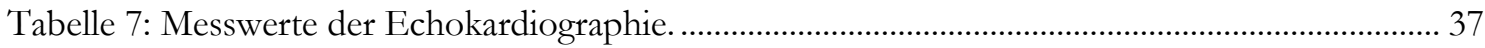

Tabelle 8: Messwerte des Herz- und Körpergewichts. ...................................................................... 39

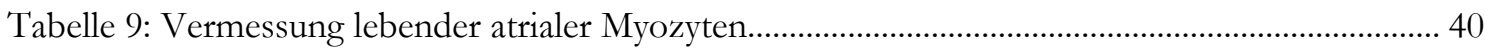

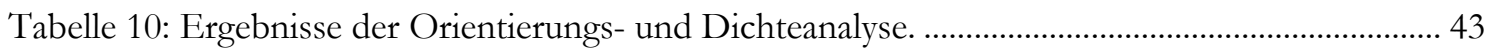

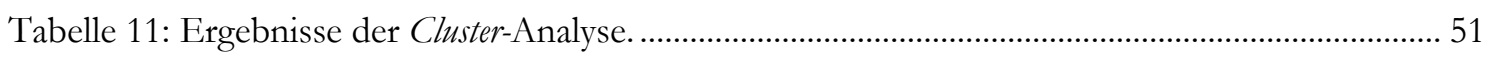

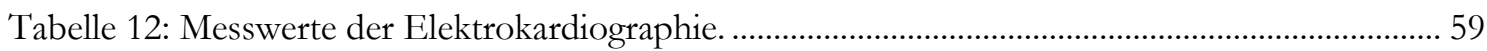




\section{Abkürzungsverzeichnis}

$2 \mathrm{p}-$

2p100mg

2p40mg

$4 \mathrm{p}-$

4p40mg

$\mathrm{AM}$

AT

AUn

AU

AWThd

BASAL

BCS

BDM

BSA

cAMP

Cav1.2

Cav3

CICR

CRU

Cx

DCM

EKG

FS

GAPDH

HCM

HCN1 zwei Wochen nach ...

zwei Wochen nach $100 \mathrm{mg} / \mathrm{kg}$ Tamoxifen (einmalige Injektion)

zwei Wochen nach $40 \mathrm{mg} / \mathrm{kg}$ Tamoxifen (einmalige Injektion)

vier Wochen nach ...

vier Wochen nach $40 \mathrm{mg} / \mathrm{kg}$ Tamoxifen (einmalige Injektion)

atriale Kardiomyozyten

axiale Tubuli

airy unit (Mikroskop)

arbitrary unit

linksventrikuläre Vorderwanddicke diastolisch

(anterior wall thickness in diastole)

EKG-Messung in Ruhe

bovines Kälberserum (bovine calf serum)

2,3-Butandionmonoxim

bovines Serumalbumin

zyklisches Adenosin-3'5'-monophosphat

spannungsabhängiger L-Typ-Ca ${ }^{2+}$-Kanal Typ 1.2

Caveolin-3

$\mathrm{Ca}^{2+}$-induzierte $\mathrm{Ca}^{2+}$-Freisetzung (calcium-induced calcium release)

$\mathrm{Ca}^{2+}$-Freisetzungseinheit (calcium release unit)

Connexin

dilatative Kardiomyopathie (dilated cardiomyopatby)

6-Kanal-Elektrokardiogramm

Flächenverkürzungsfraktion (fractional shortening)

Glyzerinaldehyd-3-phosphat-Dehydrogenase

hypertrophe Kardiomyopathie (bypertrophic cardiomyopatby)

hyperpolarisationsabhängiger zyklonukleotidregulierter Kationenkanal

Typ 1 (byperpolarization-activated cyclic nucleotide-gated channel type 1) 
HE Hämatoxylin-Eosin

i.p. intraperitoneal

ISO EKG-Messung unter $\beta$-adrenerger Stimulation durch Isoproterenol

JMC junktionaler Membrankomplex (junctional membrane complex)

JPH2 Junctophilin-2

KHK koronare Herzkrankheit

LAFS linksatriale Flächenverkürzungsfraktion (fractional shortening)

LAIDd linksatrialer Durchmesser in der Diastole (left atrial diameter in diastole)

LSM Laser-scanning-Mikroskop

LTCC spannungsabhängiger L-Typ-Ca ${ }^{2+}$-Kanal (L-type calcium channed)

LVEF linksventrikuläre Ejektionsfraktion

LVFS linksventrikuläre Flächenverkürzungsfraktion (fractional shortening)

LVIDd linksventrikulärer Durchmesser diastolisch

(left ventricular diameter in diastole)

MCM MerCreMer

MCM57 Mauslinie mit herzspezifischer Myh6-MCM-Rekombinase

MHC Myosin-Schwerkette (myosin heavy chain)

MI Myokardinfarkt

MORN membrane occupation and recognition nexus

NCX $\quad \mathrm{Na}^{+} / \mathrm{Ca}^{2+}$-Austauscher

OT oblique Tubuli

PBS phosphatgepufferte Salzlösung (phosphate-buffered saline)

PFA Paraformaldehyd

PKA Proteinkinase A

PLN Phospholamban

POST TAM Echokardiographie zwei Wochen nach Tamoxifen-Applikation (2p40mg)

PRE TAM Echokardiographie vor Tamoxifen-Applikation

pS2808 RyR2-pS2808

RNA Ribonukleinsäure (ribonucleic acid)

ROI region of interest 


$\begin{array}{ll}\text { RyR2 } & \begin{array}{l}\text { Ryanodin-Rezeptor Typ 2 } \\ \text { RyR2-pS2808 }\end{array} \\ & \begin{array}{l}\text { an der Aminosäure Serin-2808 phosphorylierter RyR2 } \\ \text { (vermittelt durch PKA) }\end{array} \\ \text { SDS } & \text { Natriumdodezylsulfat (sodium dodecyl sulfate) } \\ \text { SERCA2a } & \text { Ca }^{2+} \text {-ATPase des SR } \\ \text { SFB } & \text { Sonderforschungsbereich } \\ \text { shJPH2 } & \text { Mauslinie mit interferierender shRNA gegen JPH2 } \\ \text { shRNA } & \text { Short-hairpin-RNA } \\ \text { SR } & \text { sarkoendoplasmatisches Retikulum } \\ \text { STED } & \text { stimulated emission depletion } \\ \text { TAC } & \text { transversale Aortenkonstriktion (transverse aortic constriction) } \\ \text { TAT- } & \text { transversal-axiales Tubulus- } \\ \text { TBST } & \text { TRIS-gepufferte Salzlösung mit Tween 20 } \\ & \text { (TRIS-buffered saline with Tween 20) } \\ \text { TRIS } & \text { Tris(hydroxymethyl)aminomethan } \\ \text { T'T } & \text { transversale Tubuli } \\ \text { UMG } & \text { ventrikuläre Kardiomyozyten } \\ \text { VM } & \end{array}$




\section{$1 \quad$ Einleitung}

\subsection{Bedeutung der Herzinsuffizienz}

\subsubsection{Epidemiologie der Herzinsuffizienz}

Kardiovaskuläre Krankheiten sind der Hauptgrund für stationäre Krankenhausbehandlungen und Todesfälle in der westlichen Welt, wobei die Fallzahlen stetig zunehmen. Allein in Deutschland wurden im Jahr 2016 etwa drei Millionen Patientinnen und Patienten wegen Herz-Kreislauf-Erkrankungen (Ziffern I00 - I99 der zehnten Revision der International Statistical Classification of Diseases and Related Health Problems, ICD-10) vollstationär behandelt, davon etwa 460.000 Menschen (15,5\%) mit der Hauptdiagnose Herzinsuffizienz (I50) (Statistisches Bundesamt (Destatis) 2017). Innerhalb der Herz-Kreislauf-Erkrankungen war Herzinsuffizienz 2016 nach chronisch-ischämischer Herzkrankheit und akutem Myokardinfarkt (MI) die dritthäufigste Todesursache (etwa 40.000 Sterbefälle, 11,9\%) (Statistisches Bundesamt (Destatis) 2018). Herzinsuffizienz ist ein Krankheitsbild, das vermehrt im Alter auftritt: Der Großteil (82\%) aller vollstationären Herzinsuffizienzpatientinnen und -patienten im Jahr 2016 war mindestens 70 Jahre alt (Statistisches Bundesamt (Destatis) 2017). Aufgrund des demographischen Wandels in Deutschland wird erwartet, dass die Prävalenz der Herzinsuffizienz in Zukunft weiter steigen könnte. Allein zwischen 2000 und 2016 haben sich die Fallzahlen annähernd verdoppelt (Statistisches Bundesamt (Destatis) 2017).

\subsubsection{Entstehung der Herzinsuffizienz}

Das Herz ist als zentrale muskuläre Kreislaufpumpe in erster Linie dafür verantwortlich, frisch oxygeniertes Blut aus der Lunge über den linken Vorhof und Ventrikel in alle Körperregionen zu transportieren und umgekehrt sauerstoffarmes, mit Kohlenstoffdioxid angereichertes Blut wieder über den rechten Vorhof und Ventrikel der Lunge zum Gasaustausch zuzuführen. Als Herzinsuffizienz versteht man die Unfähigkeit des Herzens, ein diesbezüglich adäquates Blutvolumen über die Zeit auszuwerfen. Besonders häufig gehen der Herzinsuffizienz die arterielle Hypertension und/oder die koronare Herzkrankheit (KHK) voraus, außerdem der MI und die dilatative oder hypertrophe Kardiomyopathie (DCM bzw. HCM) (Wankerl et al. 1990; Ho et al. 1993; Zhang et al. 2013). Da sich das Herz über funktionelle Endarterien, die Koronarien, selbst versorgt, droht bei Gefäßverengung oder -verschluss (KHK bzw. MI) ein direkter Schaden bis unwiederbringlicher Verlust des Parenchyms, der unter Umständen deutlich früher als in von arteriellen Anastomosen durchzogenen Organen auftritt. Minderversorgtes oder untergegangenes Myokard stört die Erregungsleitung und Kontraktion, so dass sich das Schlagvolumen 
vermindert und das global benötigte Herzzeitvolumen langfristig nicht aufrechterhalten werden kann. Um den kardialen Funktionsverlust zu kompensieren, wird neben anderen Mechanismen vor allem das sympathische Nervensystem aktiviert (Brodde et al. 2006). Katecholamine wie Adrenalin setzen über kardiale G-Protein-gekoppelte $\beta_{1}$-Rezeptoren eine Signaltransduktionskette in Gang, die zunächst mittels Adenylylzyklase für die Produktion zyklischen Adenosinmonophosphats (cAMP) als second messenger sorgt (Lohse et al. 2003). Über cAMP wird die Proteinkinase A (PKA) aktiviert, welche eine Reihe an Effektorproteinen phosphoryliert, die für die elektromechanische Kopplung wesentlich sind, darunter der Ryanodin-Rezeptor Typ 2 (RyR2) und Phospholamban (PLN), welches in unphosphorylierter Form die Wiederaufnahme von Kalziumionen durch die membranständige $\mathrm{Ca}^{2+}$-ATPase des sarko(endo)plasmatischen Retikulums (SR; SERCA2a) inhibiert (Brandenburg et al. 2016a). Letztlich führen diese PKA-abhängigen Proteinmodifikationen $\mathrm{zu}$ erhöhter zytosolischer $\mathrm{Ca}^{2+}$-Konzentration und damit zu stärkerer Kontraktion und größerem Schlagvolumen (positive Inotropie). Ein weiterer Effekt aktivierter kardialer $\beta$ Rezeptoren ist die Erhöhung der Herzfrequenz (positive Chronotropie). Kompensatorisch reagiert das Herz bei Überlastung durch Druck oder Volumen zudem langfristig mit Hypertrophie der Kardiomyozyten (Göktepe et al. 2010). Gesteigerte Ino- und Chronotropie sowie Hypertrophie erhöhen wiederum den Sauerstoffbedarf des Arbeitsmyokards, sodass ein chronisches Bestehen dieses Kreislaufs die Entstehung und Progredienz der Herzinsuffizienz begünstigt.

\subsubsection{Herzinsuffizienz und atriale Dysfunktion}

Häufig geht die Herzinsuffizienz, zunächst ausgelöst durch eine ventrikuläre Pathologie, mit Funktionsstörungen der Atrien einher (Wang et al. 2003). Besonders das Vorhofflimmern ist in Patienten eine häufige Komorbidität, welche die ohnehin beeinträchtigte kardiale Funktion zusätzlich verschlechtert und für eine hohe Mortalität sorgt; konventionelle Therapien der Herzinsuffizienz allein wie zum Beispiel die Verordnung von $\beta$-Blockern versagen jedoch häufig in komorbiden Patienten, so dass bisher keine etablierte Therapie der Kombination von Herzinsuffizienz und Vorhofflimmern besteht (Benjamin et al. 2018; Wang et al. 2003; Mogensen et al. 2017; Kotecha et al. 2014). Die Prävalenz des gemeinsamen Auftretens von Herzinsuffizienz und Vorhofflimmern steigt (Heidenreich et al. 2011). Atriale Dysfunktion wurde als Auslöser kardialen Remodelings, das dem Vorhofflimmern vorausgehen kann, vorgeschlagen (Goette et al. 2017), allerdings sind die zugrundeliegenden Mechanismen bisher unzureichend erforscht (Brandenburg et al. 2016b; Guichard und Nattel 2017). Die Untersuchung der Ursachen der atrialen Dysfunktion ist daher von besonderer Bedeutung, um atrialen Krankheitsmechanismen und Komplikationen wie dem Vorhofflimmern entgegenwirken und langfristig die Prognose der Volkskrankheit Herzinsuffizienz verbessern zu können. 


\subsection{Rolle der Atrien in der Literatur}

Studien über pathologische Veränderungen am Herzen fokussieren ihre Aufmerksamkeit in der Regel auf die Ventrikel und ventrikuläre Kardiomyozyten (VM), während die Atrien und atriale Kardiomyozyten (AM) deutlich weniger untersucht wurden (Goette et al. 2017).

Im Gegensatz zu den Ventrikeln machen die Atrien an Muskelmasse zwar einen kleineren Teil des Herzens aus, funktionell gesehen sind sie aber mit dem Sitz des Sinusknotens als physiologischer Ursprung der elektrischen Herzaktion und der Koppelung venöser und arterieller Blutflüsse von großer Relevanz. Zudem hängen Ventrikelfüllung und -auswurf direkt von der atrialen Systole ab: bereits in Ruhe tragen die Atrien 10 - $20 \%$ des enddiastolischen Ventrikelvolumens bei (Rahimtoola et al. 1975), bei zwei- bis dreifach erhöhter Herzfrequenz (zum Beispiel durch Sport oder Stress) sogar 20 - 30 \% (Mitchell et al. 1965), was atrialer Kick genannt wird. Nicht zuletzt kommt den Atrien über die endokrine Sekretion von atrialem natriuretischen Peptid (ANP) bei hämodynamischem Stress eine wichtige Rolle in der Flüssigkeitshomöostase des gesamten Organismus zu (Lang et al. 1985).

\subsubsection{Besonderheiten des atrialen Tubulusnetzwerks in Säugern}

Frühe elektronenmikroskopische Studien fixierten Gewebes haben gezeigt, dass VM von Invaginationen des Sarkolemms durchzogen sind, die hauptsächlich transversal ausgerichtet und gelegentlich mit axialen Tubuli (AT) verknüpft sind und daher transversal-axiales Tubulus(TAT)-Netzwerk genannt werden (Forbes und van Neil 1988). 2-PhotonenMikroskopie ermöglichte die Untersuchung lebender VM und bestätigte diesen Fund (Soeller und Cannell 1999). Atriale Myozyten schienen kein TAT-Netzwerk (Forbes und van Neil 1988) oder höchstens rudimentäre transversale Tubuli (TT) zu besitzen (Kirk et al. 2003; Bootman et al. 2006; Smyrnias et al. 2010). Untersuchungen intakter Zellen mittels hochauflösender Stimulated-emission-depletion(STED)-Nanoskopie zeigten jedoch, dass sowohl VM als auch AM in zahlreichen Säugetierspezies unterschiedlicher Größe (Maus, Ratte, Hase, Schwein, Mensch) zellweit TAT-Netzwerke ausbilden (Wagner et al. 2012; Wagner et al. 2014; Brandenburg et al. 2018). Das atriale TAT-Netzwerk ist von geringerer Dichte als das ventrikuläre und zeichnet sich durch eine verhältnismäßig große Zahl von AT aus, die mit rund $300 \mathrm{~nm}$ einen signifikant größeren Durchmesser als TT besitzen, aber nur über wenige T'T mit dem Oberflächen-Sarkolemm verbunden sind (Brandenburg et al. 2016a). Perlschnurartig entlang diesen großvolumigen AT wurden im junktionalen SR besonders große Cluster des an der Aminosäure Serin-2808 PKA-abhängig phosphorylierten RyR2 (RyR2-pS2808) entdeckt, die nach Aktivierung von Clustern des spannungsabhängigen L-Typ-Ca ${ }^{2+}$-Kanals Typ 1.2 (Cav1.2) besonders rasch zentrale $\mathrm{Ca}^{2+}$-Transienten und $\mathrm{Ca}^{2+}$-Makrosparks erzeugen und so eine schnellere elektromechanische Kopplung als im Ventrikel ermöglichen (Brandenburg et al. 2016a). Diese hocheffektiven AT-Junktionen werden Super-hubs genannt und sind über verschiedene Spezies hinweg bis zum Menschen konserviert (Brandenburg et al. 2018). 


\subsubsection{Weitere Besonderheiten atrialer Myozyten}

Es ist bekannt, dass AM und VM unterschiedliche Genprofile aufweisen, die zu bedeutenden Unterschieden in Struktur und Funktion führen (Wu et al. 2013). Atriale Myozyten sind schmaler, ihr TAT-Netzwerk wird von AT dominiert (siehe 1.2.1) und ihr Aktionspotenzial hat eine andere Form (Thul et al. 2012; Brandenburg et al. 2016a; Wu et al. 2013). Sie kontrahieren schneller, was durch den hohen $\mathrm{Ca}^{2+}$-Gehalt des SR, ein günstiges Expressionsverhältnis von SERCA2 zu seinem Inhibitor PLN sowie die schnelle Motordomäne der $\alpha$-Myosin-Schwerkette (MHC, myosin heavy chain) erklärt werden kann (Walden et al. 2009; Lüss et al. 1999; Reiser und Kline 1998). Die kürzliche Entdeckung der effektiven $\mathrm{Ca}^{2+}$-Signaltransduktion an atrialen Super-bubs - bestehend aus der funktionellen Junktion großvolumiger zentraler AT mit großen $\mathrm{Ca}^{2+}$-Freisetzungseinheiten (CRU, calcium release unit, siehe auch 1.3.2) - bietet eine erste mechanistische Erklärung für die signifikant schnellere elektromechanische Kopplung in AM gegenüber VM (Brandenburg et al. 2016a).

Ein Hindernis bei der Untersuchung der Atrien ist die relativ aufwändige Isolation von AM. Atriales Gewebe ist im Mäuseherzen mit dem bloßen Auge schwieriger abgrenzbar und die Zahl intakter isolierter Zellen liegt im Vergleich meist unter der ventrikulären Gewebes, da die atriale Myozytenmembran leichter verletzbar ist (Brandenburg 2017). Erst moderne membranschonende Isolationsverfahren in Verbindung mit hochauflösender STED-Nanoskopie ermöglichten die Untersuchung intakter atrialer Membranstrukturen in suffizienter Stichprobengröße und -qualität (Wagner et al. 2014).

Weitere Untersuchungen der Atrien sind notwendig, um das Verständnis der Entstehung supraventrikulärer Arrhythmien, insbesondere des Vorhofflimmerns, grundlegend zu erklären. Es besteht daher ein dringender Bedarf an neuen Strategien für die Erkennung, Prävention und Behandlung der atrialen Dysfunktion als Auslöser des atrialen Remodelings. Interessanterweise wurde in Patienten eine Punktmutation des RyR2-bindenden SRProteins Junctophilin-2 (JPH2) gefunden (E169K), die sowohl im Mensch als auch im Knockin-Mausmodell durch Verschiebung der JPH2/RyR2-Stöchiometrie Vorhofflimmern begünstigen konnte (Beavers et al. 2013). Für VM ist JPH2 als wichtiges Strukturprotein etabliert, allerdings ist seine Rolle in AM bisher wenig erforscht. Aus diesen Gründen wurden in dieser Arbeit atriale Proben dahingehend untersucht, ob und wie sich Unterschiede der kardialen JPH2-Expression auf die Struktur und Funktion sowohl des atrialen Gewebes als auch der einzelnen Zellen auswirken.

\subsection{Rolle von Junctophilin-2 im Herzen}

\subsubsection{Junctophilin-2-Expressionsunterschiede im Zusammenhang mit Herzkrankheiten}

Junctophiline (Isoformen 1 bis 4) sind eine Familie hochkonservierter Proteine, die in elektrisch erregbaren Zellen zu finden sind (Landstrom et al. 2014). In Kardiomyozyten ist 
JPH2 in der Membran des SR verankert, wo es CRU-Membrankontakte mit TT bildet und die $\mathrm{Ca}^{2+}$-Signalkaskade stabilisiert (Landstrom et al. 2014). Nach der Entdeckung der Junctophilin-Familie vor beinahe 20 Jahren beschäftigten sich zahlreiche Studien mit JPH2, der Hauptisoform des Herzmuskels (Takeshima et al. 2000). Junctophilin-2 wurde als Schlüsselprotein im junktionalen Membrankomplex (JMC, junctional membrane complex), also der Verbindung von Tubulus- und SR-Membran beschrieben (siehe auch 1.3.2); seine Relevanz wurde vor allem durch JPH2-defiziente Knockout-Mäuse verdeutlicht, die nur bis zum zehnten Tag der Embryonalentwicklung, an welchem ihr Herz üblicherweise seine kontraktile Funktion aufnimmt, überlebensfähig waren (Takeshima et al. 2000). Mausmodelle von HCM, DCM und MI (Minamisawa et al. 2004; Wagner et al. 2012) sowie ein Rattenmodell von transversaler Aortenkonstriktion (TAC, transverse aortic constriction) (Xu et al. 2007) zeigten eine reduzierte Proteinexpression von JPH2 im Ventrikel. Auch in humanen Proben von Patienten mit HCM, DCM und ischämischer Kardiomyopathie wurde eine verminderte ventrikuläre JPH2-Proteinexpression festgestellt (Landstrom et al. 2011; Wu et al. 2012). Bei kardialem Stress wurden eine proteolytische Spaltung von JPH2 durch Calpain (Guo et al. 2015; Wang et al. 2018) sowie eine Herunterregulation durch mikro-Ribonukleinsäure (RNA, ribonucleic acid) beobachtet (Xu et al. 2012). Umgekehrt hatte der Knockdown von JPH2 in HL-1-Zellen in vitro eine Hypertrophie und reduzierte Amplitude des $\mathrm{Ca}^{2+}$ Transienten zur Folge (Landstrom et al. 2011), während es in vivo im Mausmodell nach Tamoxifeninduktion zu akuter Herzinsuffizienz, erhöhter Mortalität, Störung des TATNetzwerks, SR-Ca ${ }^{2+}$-Lecks und abnormalen $\mathrm{Ca}^{2+}$-Transienten kam (van Oort et al. 2011). Dagegen zeigten dreifach transgene Mäuse der Linie MerCreMer(MCM)-Short-hairpinJPH2(shJPH2, siehe auch 2.1)-Overexpression(OE), welche JPH2 konstitutiv überexprimieren, nach der Behandlung mit Tamoxifen normale Herzdimensionen und -funktion (verglichen mit Mäusen der Linie MCM als Kontrolle), außerdem wurden weder die für den JPH2-Knockdown spezifischen ultrastrukturellen Veränderungen noch abnormale $\mathrm{Ca}^{2+}$ Transienten beobachtet, sodass die JPH2-Überexpression im JPH2-Knockdown-Modell als Rescue-Ansatz nachgewiesen wurde (van Oort et al. 2011). Generell schützt die JPH2Überexpression den linken Ventrikel bei Herzinsuffizienz vor progressivem maladaptiven Remodeling (Beavers et al. 2013; Reynolds et al. 2013; Reynolds et al. 2016). Kürzlich wurde zudem in VM gezeigt, dass die Calpain-vermittelte Proteolyse von JPH2 ein N-terminales JPH2-Peptid freisetzt, welches in der Lage ist, die Transkription verschiedener Gene im Nukleus zu regulieren (Guo et al. 2018).

Die Zusammenschau der zitierten Literatur unterstreicht die wichtige Rolle, die JPH2 im Herzen (meist beschränkt auf VM) zukommt, sowohl als Stabilisator in JMC als auch möglicherweise in der transkriptionellen Regulation anderer Proteine. Erst kürzlich wurde beschrieben, dass der atrienspezifische Knockdown von JPH2 negative Auswirkungen auf die $\mathrm{Ca}^{2+}$-Homöostase von AM hat (Ni et al. 2019). Dennoch ist die subzelluläre Rolle von JPH2 im Atrium im Vergleich zum Ventrikel weitgehend unbekannt. 


\subsubsection{Junctophilin-2 als Stabilisator des junktionalen Membrankomplexes}

Die kardiale Isoform JPH2 spielt eine wichtige Rolle bei der postnatalen Ausbildung des TAT-Netzwerks in der gesunden Herzmuskelzelle und stabilisiert dyadische JMC (Beavers et al. 2014; Chen et al. 2013; Reynolds et al. 2013). Die weniger als $15 \mathrm{~nm}$ tiefe Nanodomäne des JMC bezeichnet in Kardiomyozyten den Raum zwischen den Membranen eines invaginierenden Tubulus und einer Zisterne des SR (Takeshima et al. 2000). Als Strukturprotein ist JPH2 dafür verantwortlich, den Abstand zwischen Tubulus- und SR-Membran zu stabilisieren (Takeshima et al. 2000). Während die C-terminale Transmembrandomäne im SR verankert ist, binden am zytosolischen N-Terminus acht lipophile sogenannte Membrane-occupation-and-recognition-nexus(MORN)-Domänen an Phospholipide des inneren Sarkolemm-Blatts, eine $\alpha$-helikale Domäne im Mittelteil überbrückt den junktionalen zytosolischen Raum (Beavers et al. 2014; Garbino et al. 2009; Takeshima et al. 2000). Innerhalb dieser Nanodomäne befinden sich CRU: Cluster-Formationen von spannungsabhängigen $\mathrm{Ca}^{2+}$-Kanälen (LTCC, L-type calcium channel) im Sarkolemm und direkt gegenüberliegenden RyR2-Kanälen in der SR-Membran (Franzini-Armstrong et al. 1999). Eine kardiale CRU besitzt in AM etwa sechs LTCC vom Typ Cav1.2 oder Cav1.3 und 50 RyR2, das entspricht einem Verhältnis von etwa 1:8 (Greiser et al. 2011; Carl et al. 1995). In ventrikulären CRU wird die Zahl der RyR2-Kanäle auf 35 geschätzt (Munro et al. 2016). Aktiviert ein in den Tubulus laufendes Aktionspotential die Cav1.2-Kanäle, gelangt eine geringe Menge $\mathrm{Ca}^{2+}$ in den dyadischen Spalt; da dieses zytoplasmatische Subkompartiment jedoch nur etwa einen Attoliter an Volumen fasst, resultiert eine rasche $\mathrm{Ca}^{2+}$ Konzentrationserhöhung (Prosser et al. 2010). Durch den $\mathrm{Ca}^{2+}$-Einstrom wird RyR2 aktiviert und setzt relativ große Mengen $\mathrm{Ca}^{2+}$ aus dem SR ins Zytosol frei, wofür Fabiato (1983) den Begriff $\mathrm{Ca}^{2+}$-induzierte $\mathrm{Ca}^{2+}$-Freisetzung (CICR, calcium-induced calcium release) geprägt hat. Die Kontraktion der Zelle folgt über die Bindung von $\mathrm{Ca}^{2+}$ an kontraktile Myofilamente (elektromechanische Kopplung) (Bers 2002). Im Zytosol befindliche $\mathrm{Ca}^{2+}{ }_{-}$ Ionen werden daraufhin von SERCA2a und vom $\mathrm{Na}^{+} / \mathrm{Ca}^{2+}$-Austauscher (NCX) wieder in den SR-Speicher bzw. in den extrazellulären Raum zurückbefördert, um die Relaxation der Myofilamente zu ermöglichen (Bers 2002).

Kardiomyozyten aus JPH2-Knockout-Mäusen (globaler Knockout) zeigten einen Verlust von etwa $90 \%$ der JMC im Vergleich zu Kontrolltieren, zudem wurden spontane $\mathrm{Ca}^{2+}$ Freisetzungen aus dem SR $\left(\mathrm{Ca}^{2+}\right.$-Sparks) und irreguläre $\mathrm{Ca}^{2+}$-Transienten nachgewiesen (Takeshima et al. 2000). Kardiomyozyten aus JPH2-Knockdown-Mäusen besaßen ebenfalls weniger JMC, während in verbleibenden JMC die dyadische Spalte eine größere Abstandsvariabilität zeigte (van Oort et al. 2011). Aufgrund fehlender Interaktion mit JPH2 und Entkopplung von Cav1.2 innerhalb der CRU wurden die RyR2-Cluster „hyperaktiv“ und störten durch häufige spontane Sparks die $\mathrm{Ca}^{2+}$-Homöostase (van Oort et al. 2011). JPH2 funktioniert demnach nicht nur als strukturelles Protein in JMC, sondern interagiert auch direkt funktionell mit RyR2-Kanälen. 


\subsection{Das atriale Super-hub-Modell}

Kürzlich konnte gezeigt werden, dass differenziell phosphorylierte RyR2-ClusterPopulationen in AM abhängig von der Assoziation mit dem TAT-Netzwerk exprimiert werden: Während nicht-junktionale RyR2-Cluster ohne direkten Kontakt zum TATNetzwerk einen niedrigen Phosphorylierungsstatus aufweisen, sind RyR2-Cluster in Assoziation mit AT zu einem großen Teil konstitutiv an der Aminosäure Serin-2808 PKAabhängig phosphoryliert (Brandenburg et al. 2016a). Diese posttranslationale Modifikation in situ sorgt für eine höhere $\mathrm{Ca}^{2+}$-Sensitivität der RyR2-Kanäle (Wehrens et al. 2004; Lehnart et al. 2005; Wehrens et al. 2006), die die Grundlage bilden für ein neues Modell der elektromechanischen Kopplung: Atriale Super-hubs an AT (Brandenburg et al. 2016a). Frühere Studien hatten angenommen, dass schnelle primär zentrale $\mathrm{Ca}^{2+}$-Transienten irregulär seien; stattdessen ging man von U-förmigen $\mathrm{Ca}^{2+}$-Transienten aus, also initiiert durch schnelle subsarkolemmale $\mathrm{Ca}^{2+}$-Freisetzung, welche sekundär durch zentripetale $\mathrm{Ca}^{2+}$ Diffusion über nicht-junktionale RyR2-Cluster ins Zellinnere propagiert wird (Kockskämper et al. 2001; Thul et al. 2012). Ein TAT-Netzwerk mit junktionalen RyR2-Clustern schien aufgrund der geringen atrialen Zellgröße für die elektromechanische Kopplung nicht notwendig (Dibb et al. 2009; Dibb et al. 2013). Trotzdem war bekannt, dass atriale im Vergleich zu ventrikulären Muskelpräparaten schneller kontrahieren, was mit U-förmigen $\mathrm{Ca}^{2+}$ Transienten nicht vereinbar war (Lüss et al. 1999). Brandenburg et al. (2016a) zeigten jedoch, dass $100 \%$ der untersuchten AM ein intaktes TAT-Netzwerk aufwiesen, das von AT mit einer besonders großen Oberfläche und Kontakt zum SR dominiert und von nur wenigen TT als Verbindung zum Oberflächensarkolemm durchzogen war. Perlschnurartig entlang AT wurden junktionale Cluster-couplons aus Cav1.2- und RyR2-Kanälen angelegt, die durch schnelle $\mathrm{Ca}^{2+}$-Freisetzung primär zentrale $\mathrm{Ca}^{2+}$-Transienten in $\mathrm{AM}$ erzeugten (Brandenburg et al. 2016a). In murinen AM waren zudem der hohe $\mathrm{Ca}^{2+}$-Gehalt des SR, das hohe Expressionsniveau von SERCA2a im Gegensatz zu niedrigem PLN und spontane $\mathrm{Ca}^{2+}$-Makrosparks an AT Ausdruck einer effizienten $\mathrm{Ca}^{2+}$-Freisetzung und damit schneller Kontraktilität (Brandenburg et al. 2016a; Brandenburg et al. 2018). Das ursprüngliche Modell der atrialen Super-hubs wurde in der Folge in fünf Säugtierspezies von Maus bis Mensch bestätigt, was eine wichtige Rolle der AT und der damit assoziierten Cluster-couplons in AM belegt (Brandenburg et al. 2018). Die Rolle von JPH2 für die Funktion atrialer Super-bubs wird erstmals in dieser Dissertation untersucht.

\subsection{Ziele dieser Arbeit}

Der Großteil bisheriger Studien über JPH2 konzentrierte sich auf Untersuchungen der Ventrikel und VM, obwohl bekannt ist, dass sich AM in Struktur und Funktion deutlich von VM unterscheiden. Demnach kann nur spekuliert werden, wie sich mögliche subzelluläre JPH2-Expressionsunterschiede auf die atriale Physiologie auswirken. Bisher war nicht einmal bekannt, ob die Atrien JPH2 in ähnlicher Menge exprimieren wie die Ventrikel und 
wie die atriale JPH2/RyR2-Stöchiometrie beschaffen ist. Das experimentelle Modell atrialer Super-hubs postuliert, dass $\mathrm{Ca}^{2+}$-Signale direkt an AT entstehen und dass AM über eine signifikant schnellere elektromechanische Kopplung verfügen als VM (Brandenburg et al. 2016a). In diesem Zusammenhang bleibt die Frage zu klären, ob und wie atriale Super-hubs von JPH2 abhängig sind.

Diese Arbeit soll untersuchen, ob der herzspezifische JPH2-Knockdown über Short-hairpinRNA(shRNA)-Interferenz in adulten Mäusen (van Oort et al. 2011) als Protokoll so modifiziert werden kann, dass der bekannte ventrikuläre Phänotyp einer akuten Herzinsuffizienz und die hohe Mortalität vermieden werden. Dies soll erstmals die Untersuchung der Atrien ermöglichen, die sonst durch die ausgeprägte ventrikuläre Pathologie (van Oort et al. 2011) sekundär beeinträchtigt werden. Zum Zeitpunkt der Experimente (2015 - 2017) war noch kein atrienspezifischer Promoter bekannt.

Deckt sich der bisher unbekannte atriale Phänotyp bei JPH2-Knockdown mit anderen Modellen atrialer Dysfunktion, z. B. mit dem Hypertrophiemodell nach TAC (Brandenburg et al. 2016a)? Kommt es echokardiographisch zu atrialen Kontraktionsstörungen und makroskopisch zu Veränderungen wie Hypertrophie, Fibrose oder Dilatation? Wie wirkt sich der shRNA-vermittelte JPH2-Knockown auf die Architektur des atrialen TAT-Netzwerks aus? Ändert sich die Proteinstöchiometrie im JMC? Folgt JPH2 dem Verteilungsmuster von RyR2 in Bezug auf junktionale und nicht-junktionale Cluster (Brandenburg et al. 2016a; Brandenburg et al. 2018)? Benötigt die große atriale Zahl nicht-junktionaler RyR2-Cluster eine Stabilisierung durch JPH2, auch ohne TAT-Verknüpfung mit dem Sarkolemm? Welchen Effekt hat der shRNA-vermittelte JPH2-Knockdown auf die atriale Elektrophysiologie?

Die Beantwortung dieser Fragen soll zum besseren Verständnis der atrialen Pathophysiologie beitragen. Möglicherweise können die Ergebnisse dieser Arbeit als Anstoß für neue Ansätze zur Therapie von atrialer Dysfunktion und Vorhofflimmern dienen. 


\section{Material und Methoden}

Alle Tierversuche wurden vom Tierschutzbüro der Universitätsmedizin Göttingen (UMG) und dem Niedersächsischen Landesamt für Verbraucherschutz und Lebensmittelsicherheit (LAVES) geprüft und genehmigt (Aktenzeichen Tierversuchsantrag 33.9-42502-0415/1924). Eine Auflistung der verwendeten Chemikalien, Medikamente, Färbstoffe, Antikörper, Geräte und Software befindet sich im Anhang (siehe 6).

\subsection{Transgene Mauslinien und Krankheitsmodelle}

Für die Versuche zum spezifischen Knockdown von kardialem JPH2 in einem bereits etablierten herzspezifischen Cre/loxP-Rekombinationssystem kamen die Mauslinien MCM57 (Myh6-MCM-Rekombinase) und shJPH2fl/fl (shRNA spezifisch gegen JPH2, kontrolliert durch U6-Promoter mit loxP-flankierter Neomyzin-Insertion) zum Einsatz.

Mäuse der MCM57-Linie exprimieren im Herzen konstitutiv eine durch TamoxifenBehandlung aktivierbare Cre-Rekombinase, deren Expression unter der Kontrolle des herzspezifischen $\alpha$-MHC6-Promoters steht.

Mäuse der shJPH2fl/fl-Linie besitzen ein Transgen bestehend aus dem U6-Promoter gefolgt von der gegen die JPH2-Messenger-RNA gerichtete shRNA-Sequenz. Die U6Promotersequenz ist inaktiv, da sie eine von loxP-Elementen flankierte Neomyzin-Kassette enthält, kann aber durch induzierte Cre-Rekombinase-Expression pharmakologisch aktiviert werden. In doppelt transgenen MCM57-shJPH2-Kreuzungen mit dem Cre-Transgen kann daher durch Tamoxifen-Applikation die herzspezifische Expression von JPH2-shRNA aktiviert und damit der JPH2-Knockdown induziert werden.

Beide Mauslinien wurden im C57BL/6N-Hintergrund gezüchtet und waren bereits in eine Generation über F10 hinaus zurückgekreuzt. Die Züchtung fand im Tierhaus des MaxPlanck-Instituts für Experimentelle Medizin in Göttingen statt. Gehalten wurden die Tiere anschließend in der Zentralen Tierexperimentellen Einrichtung der UMG. Die Tiere wurden gemäß europäischer Richtlinie 2010/63/EU gehalten. Eine Genehmigung für den Betrieb der Tierhaltung gemäß $\$ 11$ Tierschutzgesetz liegt vor.

Der aufgezeigte JPH2-Knockdown sollte als molekulares Modell die Entstehung einer atrialen Kardiomyopathie als Vorläufer der Herzinsuffizienz bewirken. Es ist bekannt, dass die einzelnen Mauslinien MCM57 und shJPH2fl/fl oder deren Verpaarung weder kardial noch sonstig einen pathologischen Phänotyp aufzeigen; erst die Induktion des JPH2-Knockdowns mittels Tamoxifen-Applikation führt zu phänotypischen Veränderungen (van Oort et al. 2011). 
Für die Genotypisierung mittels Polymerase-Kettenreaktion wurden folgende Primer-Paare verwendet: MCM, 5'AGGTGGACCTGATCATGGAG-3' und 5'-ATACCGGAGATCATGCAAGC-3', resultierend in ein Fragment aus 440 Basenpaaren; shJPH2, 5'-CGAAGTTATCTAGAGTCGAC-3' und 5'-GCTATGACCATGATTACGCCA-3', resultierend in ein Fragment aus 250 Basenpaaren. Die Genotypisierung wurde von Brigitte Korff und Birgit Schulz durchgeführt.

Mäuse des Genotyps MCM57-shJPH2fl/fl wurden der Gruppe „Knockdown“ zugeordnet, während die Gruppe „Kontrolle“ Mäuse des Genotyps MCM57 (und nicht Wildtyp) enthielt, um etwaige unerwünschte Effekte der Tamoxifen-induzierten Rekombinase zu kontrollieren. Gesunden Mäusen beider Gruppen wurde im Alter von neun bis zwölf Wochen einmalig entweder $40 \mathrm{mg} / \mathrm{kg}$ Körpergewicht oder $100 \mathrm{mg} / \mathrm{kg}$ Körpergewicht Tamoxifen intraperitoneal (i.p.) verabreicht. Hierzu wurden $10 \mathrm{mg}$ Tamoxifen (Sigma-Aldrich, T5648-1g) in $100 \mu \mathrm{l}$ Ethanol gelöst und mit Sojabohnenöl (Sigma-Aldrich, S7381) als Carrier-Lösung im Verhältnis 1:10 vermischt. Zur Ausbildung des Krankheitsbildes wurden entweder zwei oder vier Wochen gewährt, während derer eine professionelle Überwachung der Tiere bezüglich ihres Wohlergehens sichergestellt war. Hieraus ergaben sich drei Behandlungsprotokolle: „2p100mg“ (für zwei Wochen Induktion nach einmaliger Applikation von $100 \mathrm{mg} / \mathrm{kg}$ Tamoxifen i.p.), „4p40mg“ (für vier Wochen Induktion nach $40 \mathrm{mg} / \mathrm{kg}$ Tamoxifen i.p.) und „2p40mg“ (für zwei Wochen Induktion nach $40 \mathrm{mg} / \mathrm{kg}$ Tamoxifen i.p.). Eine „4p100mg“-Gruppe wurde aufgrund relativ hoher Mortalität in der 2p100mgGruppe nicht untersucht. Nachdem in der 2p40mg-Behandlungsgruppe sowohl fehlende Mortalität als auch proteinbiochemisch eine signifikante JPH2-Reduktion auf $42 \%$ nachgewiesen werden konnte (siehe 3.1.1), wurden alle Tiere im Anschluss ausschließlich dieser Behandlung unterzogen. Dementsprechend bezieht sich die Gruppenbezeichnung „Knockdown" in dieser Arbeit (sofern nicht anders angegeben) auf Tiere der Linie MCM57shJPH2fl/fl, die nach dem Schema 2p40mg behandelt wurden, während die Gruppe „Kontrolle“ Tiere der Linie MCM57 mit gleicher Behandlung einschließt. Abbildung 1 skizziert für 2p40mg beispielhaft den Behandlungsablauf inklusive der Untersuchungen, die vor Entnahme des Herzens durchgeführt wurden.

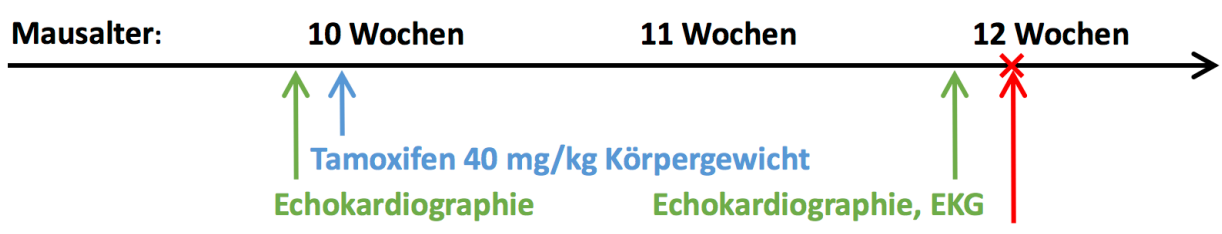

Organentnahme

\begin{abstract}
Abbildung 1: Ablauf des Behandlungsprotokolls 2p40mg.
Allen Tieren wurde intraperitoneal $40 \mathrm{mg} / \mathrm{kg}$ Körpergewicht Tamoxifen verabreicht. Echokardiographische Untersuchungen fanden einen Tag vor der Tamoxifen-Applikation und einen Tag vor der Tötung statt, zusätzlich eine elektrokardiographische Untersuchung (EKG) einen Tag vor der Tötung. Zwei Wochen nach Tamoxifen-Applikation wurde das Herz entnommen.
\end{abstract}




\subsection{Proteinbiochemische Analyse mittels Western-Blot}

Nach Anästhesie der mit Tamoxifen behandelten Mäuse durch ein Gemisch aus Sauerstoff $\left(\mathrm{O}_{2}\right)$ und 1,5\% Isofluran wurde der Tod durch Genickbruch herbeigeführt. Nach Öffnung des Brustkorbs wurde das Herz rasch entnommen, in eine gekühlte phosphatgepufferte Salzlösung (PBS, phosphate-buffered saline) überführt und nass gewogen, wonach der Aortenstumpf auf eine stumpfgeschliffene 21-G-Kanüle gesetzt und dort fixiert wurde. Das Ende der Kanüle sollte die Aortenklappe nicht passieren, um die spätere Koronarperfusion zu gewährleisten. Mithilfe eines modifizierten Langendorff-Systems wurde das Herz für eine Minute bei einer Flussrate von $4 \mathrm{ml} / \mathrm{min}$ und $37{ }^{\circ} \mathrm{C}$ mit PBS perfundiert, um vorhandene Blutreste aus dem Gefäßsystem zu entfernen. Anschließend wurde das Herz in PBS unter Zuhilfenahme eines Binokulars manuell seziert, wobei beide Atrien inklusive der Auriculae cordis entnommen und in flüssigem Stickstoff schockgefroren wurden. Ebenso wurde nach Entfernung des rechten Ventrikels mit dem linksventrikulären Gewebe verfahren.

Die Proben wurden bei $4{ }^{\circ} \mathrm{C}$ mittels eines Dispergiergeräts (MICCRA D-1, MICCRA $\mathrm{GmbH}$ ) in Homogenisierungspuffer (siehe Tabelle 1 für alle in diesem Kapitel verwendeten Lösungen) homogenisiert, 30 Minuten im Überkopfschüttler bei $4{ }^{\circ} \mathrm{C}$ belassen, zehn Minuten lang bei $5000 \mathrm{~g}$ und $4{ }^{\circ} \mathrm{C}$ zentrifugiert, woraufhin der Überstand aliquotiert und in flüssigem Stickstoff schockgefroren wurde.

Zur Proteinmengenbestimmung mithilfe des Pierce-BCA-Protein-Assay \#23227 (Thermo Fisher Scientific) wurde das Homogenat im Verhältnis 1:20 mit Wasser $\left(\mathrm{H}_{2} \mathrm{O}\right)$ verdünnt, in einer Mikrotiterplatte gemäß den Angaben des Herstellers mit den Assay-Reagenzien vermischt und 30 Minuten bei $37^{\circ} \mathrm{C}$ inkubiert. Die Ergebnisse der Photometrie wurden mit einem Spektrophotometer (BioTek) ermittelt und mit dem Programm KC4 ${ }^{\mathrm{TM}}$ (BioTek) ausgewertet.

Für die Auftrennung der Proteine wurden Zweiphasengele (6\%/12\%, siehe Tabelle 1) manuell gegossen. Die Elektrophorese erfolgte in 1x Natriumdodezylsulfat(SDS, sodium dodecyl sulfate)-Laufpuffer bei $70 \mathrm{~V}$. Der Lauf wurde gestoppt, als die erste Bande des vorgefärbten Molekulargewichtmarkers den unteren Gelbereich erreichte. In einem nassen BlotApparat wurden die Proteine anschließend auf eine Nitrozellulosemembran übertragen. Hierzu wurden Blot-Module bestehend aus einem Schwämmchen, zwei Filterpapieren, einer Nitrozellulosemembran, dem Proteingel, zwei Filterpapieren und einem weiteren Schwämmchen in Transferpuffer eingelegt und die Proteine anschließend bei konstant $100 \mathrm{~V}$ und $4{ }^{\circ} \mathrm{C}$ transferiert. Die Überprüfung des gleichmäßigen Proteintransfers erfolgte mittels Ponceau-Färbung der Nitrozellulosemembran. Um unspezifische Proteinbindungen zu verhindern, wurde die Membran daraufhin für eine Stunde bei Raumtemperatur mit $5 \%$ Milchpuffer behandelt.

Die Primär- und Sekundärantikörper (siehe 6.4.1 und 6.4.2 im Anhang) wurden simultan in $5 \%$ Milchpuffer verdünnt und bei $4{ }^{\circ} \mathrm{C}$ über Nacht zur Bindung der Zielproteine auf der Membran belassen, bevor die ungebundenen Antikörper am Folgetag in vier Waschschrit- 
ten zu je zehn Minuten mittels Tris(hydroxymethyl)aminomethan(TRIS)-gepufferter Salzlösung mit Tween20 (TBST, TRIS-buffered saline with Tween20) entfernt wurden.

Zur Detektion und Analyse der Fluoreszenzbanden wurde eine LI-COR Odyssey ${ }^{\circledR}$ FcxKamera mit zugehöriger Software (Image Studio ${ }^{\mathrm{TM}}$ ) verwendet. Für die Aufnahmen der Abbildung 2 (siehe 3.1) wurde eine Kodak Imaging Station 4000R PRO (Carestream Health Inc.) verwendet, die Dichte der Banden wurde mit MI Software V.5.0.3 (Carestream Health Inc.) gemessen.

Tabelle 1: Lösungen, Puffer und Gele für Western-Blots.

\begin{tabular}{|c|c|c|}
\hline Lösung & Zusammensetzung & \\
\hline \multirow[t]{11}{*}{ Homogenisierungspuffer } & HEPES & $10 \mathrm{mM}$ \\
\hline & Saccharose & $300 \mathrm{mM}$ \\
\hline & $\mathrm{NaCl}$ & $150 \mathrm{mM}$ \\
\hline & EGTA & $1 \mathrm{mM}$ \\
\hline & $\mathrm{CaCl}_{2}$ & $2 \mathrm{mM}$ \\
\hline & $\mathrm{pH}$ & 7,4 \\
\hline & PhosSTOPTм & 1 Tablette $/ 10 \mathrm{ml}$ \\
\hline & (Phosphatase-Inhibitor-Cocktail) & \\
\hline & cOmplete ${ }^{\mathrm{TM}}$, Mini, EDTA free & 1 Tablette $/ 10 \mathrm{ml}$ \\
\hline & (Protease-Inhibitor-Cocktail) & \\
\hline & Triton X-100 & $1 \%$ \\
\hline Trenngel $6 \%$ & Acrylamid/Bisacrylamid & $1,2 \mathrm{ml}$ \\
\hline \multirow[t]{4}{*}{ (Ansatz für 6 ml) } & 4x TRIS/SDS, pH 8,8 & $1,5 \mathrm{ml}$ \\
\hline & $\mathrm{H}_{2} \mathrm{O}$ & $3,3 \mathrm{ml}$ \\
\hline & 10\% APS-Lösung & $24 \mu \mathrm{l}$ \\
\hline & TEMED & $9 \mu \mathrm{l}$ \\
\hline Trenngel $12 \%$ & Acrylamid/Bisacrylamid & $2,4 \mathrm{ml}$ \\
\hline \multirow[t]{4}{*}{ (Ansatz für 6 ml) } & 4x TRIS/SDS, pH 8,8 & $1,5 \mathrm{ml}$ \\
\hline & $\mathrm{H}_{2} \mathrm{O}$ & $2,1 \mathrm{ml}$ \\
\hline & 10\% APS-Lösung & $24 \mu \mathrm{l}$ \\
\hline & TEMED & $9 \mu \mathrm{l}$ \\
\hline Sammelgel & Acrylamid/Bisacrylamid & $1 \mathrm{ml}$ \\
\hline \multirow[t]{2}{*}{ (Ansatz für 2 Gele, 7,5 ml) } & 4x TRIS/SDS, pH 6,8 & $1,88 \mathrm{ml}$ \\
\hline & $\mathrm{H}_{2} \mathrm{O}$ & $4,62 \mathrm{ml}$ \\
\hline
\end{tabular}




\begin{tabular}{|c|c|c|}
\hline Lösung & Zusammensetzung & \\
\hline Sammelgel & 10\% APS-Lösung & $37,5 \mu \mathrm{l}$ \\
\hline (fortgesetzt) & TEMED & $15 \mu 1$ \\
\hline \multirow[t]{3}{*}{$4 \times$ TRIS $/$ SDS, $\mathrm{pH}=6,8$} & TRIS & $500 \mathrm{mM}$ \\
\hline & SDS & $0,4 \%$ \\
\hline & $\mathrm{pH}$ & 6,8 \\
\hline \multirow[t]{3}{*}{$4 \mathrm{x}$ TRIS/SDS, $\mathrm{pH}=8,8$} & TRIS & $1,5 \mathrm{M}$ \\
\hline & SDS & $0,4 \%$ \\
\hline & $\mathrm{pH}$ & 8,8 \\
\hline \multirow[t]{4}{*}{ 10x SDS-Laufpuffer } & TRIS & $250 \mathrm{mM}$ \\
\hline & Glycin & $1,9 \mathrm{M}$ \\
\hline & SDS & $1 \%$ \\
\hline & $\mathrm{pH}$ & 8,3 \\
\hline \multirow[t]{2}{*}{ 10x Transferpuffer } & TRIS & $325 \mathrm{mM}$ \\
\hline & Glycin & $1,9 \mathrm{M}$ \\
\hline \multirow[t]{2}{*}{ 1x Transferpuffer } & 10x Transferpuffer & $10 \%$ \\
\hline & Methanol & $20 \%$ \\
\hline \multirow[t]{2}{*}{ Ponceau S Solution } & Ponceau S & $0,5 \%$ \\
\hline & Essigsäure & $10 \%$ \\
\hline \multirow[t]{4}{*}{ 10x TBST-Puffer } & TRIS & $100 \mathrm{mM}$ \\
\hline & $\mathrm{NaCl}$ & $1,5 \mathrm{M}$ \\
\hline & $\mathrm{pH}$ & 7,5 \\
\hline & Tween ${ }^{\circledR} 20$ & $0,1 \%$ \\
\hline \multirow[t]{2}{*}{$5 \%$ Milchpuffer } & Milchpulver & $5 \%$ \\
\hline & 10x TBST-Puffer & $10 \%$ \\
\hline
\end{tabular}

\subsection{Echokardiographie}

Um phänotypische und funktionelle Veränderungen des Herzmuskels durch die Tamoxifenbehandlung festzustellen, wurden zu zwei Zeitpunkten echokardiographische Daten (30-MHz-Schallkopf: MS400 MicroScan ${ }^{\text {TM }}$ Transducer, Vevo ${ }^{\circledR} 2100$, VisualSonics) erhoben: Zunächst einen Tag vor der geplanten Injektion von Tamoxifen und ferner zwei Wo- 
chen danach, entsprechend einem Tag vor Euthanasie der Versuchstiere. Die untersuchten Parameter sind in Tabelle 2 dargestellt.

Tabelle 2: Morphologische Parameter der Echokardiographie.

\begin{tabular}{ll}
\hline \hline Parameter & Abkürzung \\
\hline Durchmesser des linken Atriums in der Diastole & LAIDd \\
Durchmesser des linken Atriums in der Systole & LAIDs \\
Durchmesser des linken Ventrikels in der Diastole & LVIDd \\
Durchmesser des linken Ventrikels in der Systole & LVIDs \\
Endokardiale Fläche des linken Ventrikels in der Diastole & Area d \\
Endokardiale Fläche des linken Ventrikels in der Systole & Area s \\
Lange Achse (Aortenklappe bis Herzspitze) in der Diastole & Ld \\
Lange Achse (Aortenklappe bis Herzspitze) in der Systole & Ls \\
Vorderwanddicke des linken Ventrikels in der Diastole & AWThd \\
\hline \hline
\end{tabular}

Aus diesen morphologischen Parametern lassen sich funktionelle Werte errechnen, die in Tabelle 3 aufgeführt sind.

Tabelle 3: Funktionelle Parameter der Echokardiographie.

\begin{tabular}{ll}
\hline Parameter & Abkürzung und Formel \\
\hline Ejektionsfraktion des linken Ventrikels $(\%)$ & LVEF $=\frac{\text { Vold }- \text { Vol } s}{\text { Vold }} \times 100$ \\
Verkürzungsfraktion des linken Atriums (\%) & LAFS $=\frac{\text { LAIDd }- \text { LAIDs }}{\text { LAIDd } \times 100}$ \\
Verkürzungsfraktion des linken Ventrikels $(\%)$ & LVFS $=\frac{\text { LVIDd }- \text { LVIDs }}{\text { LVIDd } \times 100}$ \\
Volumen des linken Ventrikels in der Diastole $(\mu l)$ & Vold $=\frac{5}{6} \times$ Aread $\times$ Ld \\
Volumen des linken Ventrikels in der Systole $(\mu l)$ & Vol $s=\frac{5}{6} \times$ Area $\times$ Ls \\
\hline \hline
\end{tabular}

Die Echokardiographie erfolgte in Narkose mit 1,5\% Isofluran in Sauerstoff $(1 \mathrm{l} / \mathrm{min})$ über eine Nasenmaske. Die Tiere wurden in Narkose mittels Enthaarungscreme am frontalen Thorax enthaart, um das transthorakale Echo-Schallfenster (lange Achse links parasternal auf der Höhe der Aortenklappe) zu ermöglichen. Die Untersuchungen wurden von Roland Blume und Marcel Zoremba der Sonderforschungsbereich(SFB)-Service-unit S01 der Abteilung Kardiologie und Pneumologie der UMG durchgeführt, wonach die Daten von 
Beate Knocke aus derselben Abteilung ausgewertet wurden. Sowohl die echokardiographischen Untersuchungen als auch die Analyse der Daten fanden als Blindversuch statt.

\subsection{Messung von Herz- und Körpergewicht}

Bei jedem Versuchstier wurden nach Euthanasie und Herzentnahme manuell mittels Waage das Herz- und Körpergewicht ermittelt. Die Daten wurden in Microsoft Excel importiert und zueinander ins Verhältnis gesetzt.

\subsection{Zellisolation}

Die Isolation von AM und VM zwei Wochen nach Tamoxifenbehandlung erfolgte zunächst wie in Bezug auf die proteinbiochemische Analyse dargelegt (siehe 2.2), wobei das kanülierte Herz initial für vier Minuten bei einer Flussrate von $4 \mathrm{ml} / \mathrm{min}$ und $37^{\circ} \mathrm{C}$ mit $\mathrm{Ca}^{2+}$-freiem Perfusionspuffer (siehe

Tabelle 4 für die Zusammensetzung aller verwendeten Lösungen) perfundiert wurde. Dieser Puffer enthält den Myosin-ATPase-Inhibitor 2,3-Butandionmonoxim (BDM, SigmaAldrich, B0753) um Myokardkontraktionen zu unterbinden. Nach Überprüfung der korrekten Perfusion auch der Atrien wurde das Herz anschließend für weitere neun Minuten bei gleicher Flussrate und Temperatur mit Verdaupuffer, der Typ-II-Kollagenase (ca. $300 \mathrm{U} / \mathrm{mg})$ und $40 \mu \mathrm{M}$ Kalziumchlorid $\left(\mathrm{CaCl}_{2}\right)$ enthält, perfundiert. Danach wurde das Herz in Verdaupuffer unter Zuhilfenahme eines Binokulars manuell seziert, wobei beide Atrien inklusive der Auriculae cordis entnommen und grob zerkleinert wurden. Um einzelne Zellen aus ihrem Verband zu lösen, wurde die Lösung in einer 1-ml-Pipette etwa eine Minute lang vorsichtig auf- und abbewegt, wonach der Verdau durch Zugabe von Stopppuffer, der bovines Kälberserum (BCS, bovine calf serum) und $\mathrm{CaCl}_{2}$ enthält, beendet wurde. Zur Aufreinigung der Zellsuspension wurde diese nach Entfernung grober Gewebsbrocken eine Minute lang bei $46 \mathrm{~g}$ zentrifugiert. Anschließend wurde der Überstand verworfen und das Zellpellet in Stopppuffer resuspendiert. Simultan wurde nach Entfernung des rechten Ventrikels auch das linksventrikuläre Gewebe zunächst grob zerkleinert und anschließend in einer 5-ml-Pipette auf- und abbewegt, bevor der Verdau durch Stopppuffer beendet wurde. Zur Aufreinigung der Suspension wurden die Zellen acht Minuten lang zum Zwecke der Sedimentation stehen gelassen, der Überstand verworfen und das Zellpellet in Stopppuffer resuspendiert. Das weitere Vorgehen unterschied sich je nach Art des nachfolgenden Versuchs und wird an den entsprechenden Stellen beschrieben. Alle für Experimente benutzten Kardiomyozyten wurden einer Qualitätskontrolle unterzogen (keine Membrandefekte oder aberrante Kontraktionen, reguläre Querstreifung wie beschrieben von Wagner et al. 2014). Regelmäßige Zellzählungen in einer Fuchs-Rosenthal-Kammer (0,2 mm Tiefe, $0,0625 \mathrm{~mm}^{2}$ Fläche) nach Trypanblau-Färbung zeigten ein beständiges Mittel von $10.000 / \mathrm{ml}$ in der AM-Suspension. 
Tabelle 4: Pufferlösungen für die Kardiomyozytenisolation.

\begin{tabular}{|c|c|c|c|}
\hline 10x Perfusionspuffer (Stock) & $\begin{array}{l}\text { Endkonzentration } \\
(\mathrm{mM}) \text { in } 1 \mathrm{x} \text { Puffer }\end{array}$ & $\begin{array}{l}\text { Molekulargewicht } \\
(\mathrm{g} / \mathrm{mol})\end{array}$ & Menge \\
\hline $\mathrm{NaCl}$ & 120,4 & 58,44 & $70,3 \mathrm{~g}$ \\
\hline $\mathrm{KCl}$ & 14,7 & 74,56 & $11,0 \mathrm{~g}$ \\
\hline $\mathrm{KH}_{2} \mathrm{PO}_{4}$ & 0,6 & 136,09 & $0,82 \mathrm{~g}$ \\
\hline $\mathrm{Na}_{2} \mathrm{HPO}_{4} \times 2 \mathrm{H}_{2} \mathrm{O}$ & 0,6 & 177,99 & $1,1 \mathrm{~g}$ \\
\hline $\mathrm{MgSO}_{4} \times 7 \mathrm{H}_{2} \mathrm{O}$ & 1,2 & 246,48 & $3,0 \mathrm{~g}$ \\
\hline HEPES & 10,0 & 238,31 & $23,83 \mathrm{~g}$ \\
\hline $\mathrm{H}_{2} \mathrm{O}$ & - & - & ad 1,01 \\
\hline $\mathrm{pH}$-Wert 7,4 bei $37^{\circ} \mathrm{C}$ & - & - & - \\
\hline 1x Perfusionspuffer & Endkonzentration & $\begin{array}{l}\text { Molekulargewicht } \\
(\mathrm{g} / \mathrm{mol})\end{array}$ & Menge \\
\hline 10x Perfusionspuffer & $1 \mathrm{x}$ & - & $50 \mathrm{ml}$ \\
\hline $\mathrm{NaHCO}_{3}$ & $4,6 \mathrm{mM}$ & 84,01 & $0,195 \mathrm{~g}$ \\
\hline Taurin & $30 \mathrm{mM}$ & 125,2 & $1,875 \mathrm{~g}$ \\
\hline 2,3-Butandionmonoxim (BDM) & $10 \mathrm{mM}$ & 101,1 & $0,5 \mathrm{~g}$ \\
\hline Glukose & $5,5 \mathrm{mM}$ & 180,16 & $0,5 \mathrm{~g}$ \\
\hline $\mathrm{H}_{2} \mathrm{O}$ & - & - & ad $500 \mathrm{ml}$ \\
\hline $\mathrm{pH}$-Wert 7,4 bei $37^{\circ} \mathrm{C}$ & - & - & - \\
\hline Verdaupuffer & Endkonzentration & Stock-Konzentration & Menge \\
\hline Kollagenase Typ II & $300 \mathrm{U} / \mathrm{ml}$ & $300 \mathrm{U} / \mathrm{ml}$ & $0,1 \mathrm{~g}$ \\
\hline $\mathrm{CaCl}_{2}$ & $40 \mu \mathrm{M}$ & $100 \mathrm{mM}$ & $20 \mu \mathrm{l}$ \\
\hline 1x Perfusionspuffer & - & - & ad $50 \mathrm{ml}$ \\
\hline Stopppuffer & Endkonzentration & Stock-Konzentration & Menge \\
\hline BCS & $10 \%$ & $100 \%$ & $5 \mathrm{ml}$ \\
\hline $\mathrm{CaCl}_{2}$ & $12,5 \mu \mathrm{M}$ & $100 \mathrm{mM}$ & $6,25 \mu \mathrm{l}$ \\
\hline 1x Perfusionspuffer & - & - & ad $50 \mathrm{ml}$ \\
\hline
\end{tabular}

\subsection{Konfokalmikroskopische Aufnahmen lebender Kardiomyozyten}

Zur Mikroskopie Knockdown-spezifischer Veränderungen der Architektur des atrialen TATNetzwerkes wurde ein konfokales Laser-scanning-Mikroskop (LSM710, PlanApochromat 63x/1.4 NA Öl-Objektiv, ZEISS) inklusive der Software des Herstellers 
(ZEN 2009) verwendet. Die Aufnahmen wurden im LSM-Dateiformat gespeichert, wobei die Pixelgröße auf $100 \mathrm{~nm}$ x $100 \mathrm{~nm}$ bei einer Verweildauer von 0,5 - 0,6 $\mu$ s pro Bildpunkt festgelegt wurde. Jede Zelle wurde zunächst zur Beurteilung der Qualität und zur späteren Vermessung durchlichtmikroskopisch aufgenommen.

\subsubsection{Live-Membranfärbung mittels di-8-ANEPPS}

Der lipophile Farbstoff di-8-ANEPPS ist in der Lage, Plasmamembranen lebender Kardiomyozyten zu färben; dies schließt auch das intrazelluläre TAT-Netzwerk mit ein. Hierzu wurden zunächst AM nach obigem Protokoll (siehe 2.5) isoliert und nach der Aufreinigung nochmals zehn Minuten zur Sedimentation stehen gelassen. Nach Entfernung des Überstands wurde das Zellpellet in $400 \mu \mathrm{l}$ di-8-ANEPPS $(50 \mu \mathrm{M}$ in Perfusionspuffer, Invitrogen, D3167) resuspendiert und umgehend auf ein mit $2 \mu \mathrm{l}$ Laminin $(2 \mathrm{mg} / \mathrm{ml})$ beschichtetes Deckglas (Ø $42 \mathrm{~mm}$ ) gegeben, um den Zellen das Absetzen schon während des Färbevorgangs zu ermöglichen. Nach fünfzehnminütiger Inkubation im Dunkeln bei Raumtemperatur wurde überflüssiger Farbstoff mit Perfusionspuffer ausgewaschen, bevor das Deckglas mit $1 \mathrm{ml}$ Perfusionspuffer bedeckt wurde. Die konfokalmikroskopischen Aufnahmen wurden innerhalb eines Zeitfensters von bis zu drei Stunden nach Zellisolation erstellt.

Die Anregung erfolgte bei $488 \mathrm{~nm}$ Wellenlänge, während im Bereich von $535-759 \mathrm{~nm}$ detektiert wurde. Die Laserleistung (power) betrug $5 \%$ und die Signalverstärkung (gain) 800 - 950. Die Lochblendenöffnung von 1 AUn (airy unit) resultierte in einer optischen Scheibendicke von $900 \mathrm{~nm}$.

\subsection{Analyse der konfokalen Aufnahmen lebender Kardiomyozyten}

Sämtliche Auswertungen der konfokalen Bilder erfolgten mit der frei erhältlichen Software Fiji (https://fiji.sc), die auf ImageJ (https://imagej.nih.gov/ij/) basiert. Hierzu wurden die LSM-Dateien importiert und in das TIF-Format umgewandelt, um sie zu bearbeiten und auszuwerten. Die endgültige Organisation und Analyse der Daten wurden in Excel vorgenommen.

\subsubsection{Zellgrößenbestimmung}

Die Bestimmung der Zellgrößen erfolgte, wie oben beschrieben, an Durchlichtaufnahmen lebender AM. Die Umrisse jeder Zelle wurden manuell als region of interest (ROI) abgesteckt und dem ROI-Manager hinzugefügt. Das Programm errechnet daraufhin semi-automatisch die Parameter Fläche, längste Achse (Länge) und kürzeste Achse (Breite). Zusätzlich wurden die Werte für den Quotienten aus Länge und Breite berechnet. 


\subsubsection{Dichte und Orientierung des TAT-Netzwerks}

Um das TAT-Netzwerk via Fiji vermessen zu können, wurde ein von der AG Lehnart entwickeltes standardisiertes quantitatives Analyseprotokoll verwendet und in geringem Umfang adaptiert (Wagner et al. 2014). Zunächst wurde bei jeder Zelle eine ROI festgelegt, die so viele Bestandteile des intrazellulären TAT-Netzwerks wie möglich enthielt, wobei die äußere Plasmamembran und der Zellkern ausgespart wurden. Anschließend wurde folgende Befehlabfolge angewendet, um den Kontrast des di-8-ANEPPS-Signals zu erhöhen und eine Binärdatei zu erzeugen, die nur noch zwischen den Informationen „Signal“ (Tubulusmembran) und ,kein Signal“ (keine Tubulusmembran) unterscheidet:

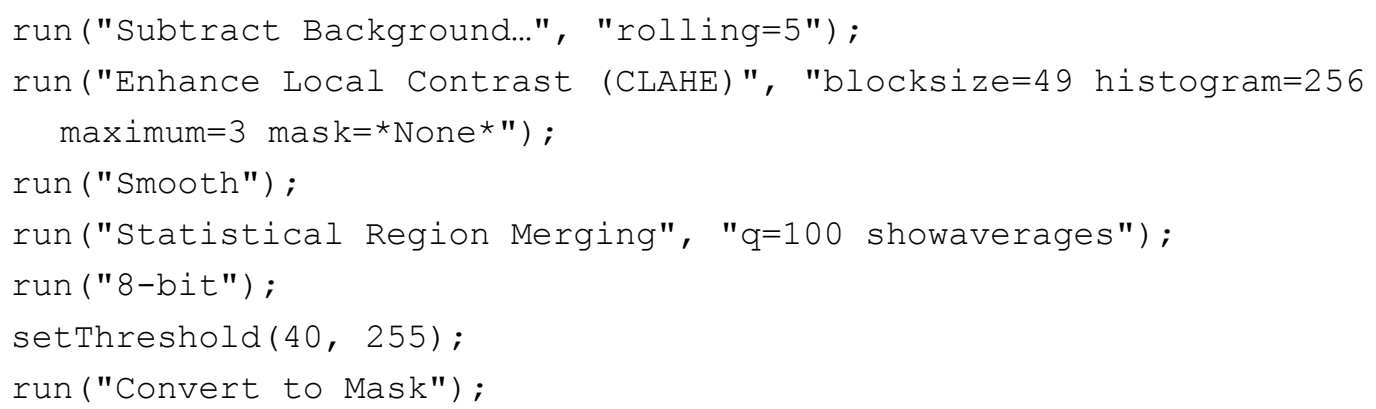

Das entstandene Binärbild des TAT-Netzwerks wurde nun mit den nachfolgenden Befehlen modifiziert, die die sogenannte Skeletonisierung des Signals und anschließende Analyse des Skeletts ermöglichen. Skeletonisierung (oder Thinning) beschreibt in diesem Fall die Reduktion einer geometrischen 2D-Figur auf ihre Hauptachse zum Zwecke der leichteren Auswertung (Lam et al. 1992).

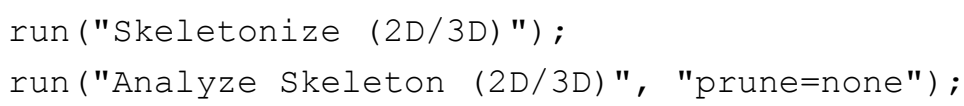

Das Plugin der Skelettanalyse stellt Informationen wie Astanzahl und -länge sowie Anzahl der Knotenpunkte des Netzwerks bereit. Durch Aufsummierung der Länge aller Äste erhält man die Länge des TAT-Netzwerks einer Zelle. Über den ROI-Manager wurde, wie oben am Durchlichtbild beschrieben, die Fläche des TAT-Netzwerks berechnet. Um die Dichte des TAT-Netzwerks bezogen auf die Zellgröße zu berechnen, wurde also der Quotient aus der Länge des TAT-Netzwerks $(\mu \mathrm{m})$ und der Fläche desselben $\left(\mu \mathrm{m}^{2}\right)$ gebildet.

Das skeletonisierte Bild war auch die Grundlage für die Messung der Orientierung oder Ausrichtung einzelner Elemente des TAT-Netzwerks. Mithilfe des Befehls

run("Directionality", "method=[Fourier components] nbins=180 histogram=-45 display_table");

wurde für jedes Zellskelett ein Histogramm erstellt, welches die Häufigkeit der Elemente einer bestimmten Ausrichtung in Relation zur longitudinalen Zellachse darstellt, wobei die Klassengröße auf $1^{\circ}$ festgelegt wurde. Dementsprechend wurden TT unter $90^{\circ}$ zusammengefasst, AT unter $0^{\circ}$ und oblique Tubuli (OT) unter $\pm 45^{\circ}$. Aus Gründen der Symmetrie stellen die Histogramme nur die Werte von $0^{\circ}$ bis $179^{\circ}$ dar $\left(0^{\circ}\right.$ und $180^{\circ}$ wurden als gleichwertig angesehen und daher nicht doppelt gezählt, ebenso $1^{\circ}$ und $181^{\circ}$ und alle 
weiteren). Um die zweigipflige Verteilung der Tubulusausrichtung bestmöglich zu zeigen, wurde die Abszisse mit Werten von $-45^{\circ}$ bis $134^{\circ}$ beschriftet, wobei der Bereich von $-45^{\circ}$ bis $-1^{\circ}$ dem Bereich von $135^{\circ}$ bis $179^{\circ}$ entspricht. Aus allen Histogrammdaten innerhalb der Kontroll- bzw. Knockdown-Gruppe wurden Mittelwerte für jeden Winkelgrad gebildet, aus denen sich ein repräsentatives Histogramm pro Gruppe ergab.

Um nicht nur die relative Verteilung von AT, TT und OT innerhalb einer Zelle, sondern auch die absolute Dichte der jeweiligen Elemente vergleichen zu können, wurde für AT der Prozentsatz des Bereiches von $90 \pm 3^{\circ}$ festgelegt, analog für TT von $0 \pm 3^{\circ}$ und für OT von $\pm 45 \pm 3^{\circ}$. Für jede Aufnahme wurden innerhalb des jeweiligen Bereichs die prozentualen Anteile dieser jeweils sieben Winkelgrade aufsummiert, mit der Gesamtlänge des Skeletts dieser Aufnahme multipliziert und zuletzt durch die Fläche des Skeletts geteilt. Die gemittelten Werte wurden zwischen der Kontroll- und Knockdown-Gruppe verglichen.

\subsection{Histologie}

Für histologische Untersuchungen wurden Herzen wie oben beschrieben (siehe 2.2) nach zweiwöchiger Tamoxifenbehandlung entnommen, jedoch nicht gespült, sondern direkt zur Fixierung über Nacht in $5 \mathrm{ml}$ Roti®-Histofix $4 \%$ (phosphatgepufferte Formaldehydlösung, Roth, P087) eingelegt, nachdem die Herzspitze zur besseren Einstellung der optimalen Schnittebene entfernt worden war. Anschließend wurden die Herzen im Einbettautomaten mittels einer aufsteigenden Alkoholreihe entwässert und in einen Paraffinblock eingebettet. Nach dem Erkalten der Blöcke wurden die Proben am Mikrotom in Scheiben von $4 \mu \mathrm{m}$ Dicke geschnitten und getrocknet, um sie daraufhin zu färben. Alle bis hierhin beschriebenen Schritte wurden von Birgit Schumann durchgeführt.

Von den gefärbten und getrockneten Schnitten wurden zunächst Übersichtsaufnahmen an einem Binokular und dann Detailaufnahmen (40x) des atrialen Endo- und Epikards sowie des interatrialen Septums und des atrialen interstitiellen Raums mittels einer Mikroskopkamera (Axiocam 105 Color, Software ZEN 2009, ZEISS) angefertigt.

\subsubsection{Hämatoxylin-Eosin(HE)-Färbung}

Zur Qualitätskontrolle und allgemeinen Beurteilung der Gewebsarchitektur wurden HEFärbungen von Schnitten auf Höhe der Aortenklappe inklusive beider Atrien angefertigt. Die Schnitte wurden mit Roticlear ${ }^{\circledR}$ (Xylol, Roth, A538.5) entparaffiniert und in einer absteigenden Alkoholreihe rehydriert, um sie auf den wässrigen Kernfarbstoff vorzubereiten. Anschließend folgte die Färbung mit Hämatoxylin (Mayers Hämatoxylinlösung, SigmaAldrich, 039K4367) und Eosin (Eosin Y Lösung alkoholisch, Sigma-Aldrich, 118K4355), woraufhin die Proben wieder in einer aufsteigenden Alkoholreihe entwässert und mit Roti ${ }^{\circledR}$-Histokitt (synthetisches Einschlussmittel, Roth, 6638.1) eingebettet wurden. 


\subsubsection{Sirius-Red/Fast-Green-Färbung}

Zur Feststellung eventueller fibrotischer Umbauvorgänge wurden Sirius-Red/Fast-GreenFärbungen von Schnitten auf Höhe der Aortenklappe inklusive beider Atrien angefertigt. Die Schnitte wurden mit Roticlear ${ }^{\circledR}$ entparaffiniert und in einer absteigenden Alkoholreihe rehydriert. Anschließend folgte die Färbung mit Sirius Red (0,1 g Direct Red 80 in $100 \mathrm{ml}$ $1 \%$ Pikrinsäure, Sigma-Aldrich, 365548-5g) und Fast Green (0,1 \% Fast Green FCF in $\mathrm{H}_{2} \mathrm{O}$, Roth, 0301.1-25g), woraufhin die Proben wieder in einer aufsteigenden Alkoholreihe entwässert und mit Roti®-Histokitt eingebettet wurden.

\subsection{Konfokalmikroskopische und STED-Aufnahmen fixierter Kardiomyozyten mittels Immunfluoreszenzfärbung}

Für die Darstellung Knockdown-spezifischer Veränderungen auf der Ebene von atrialer Proteinexpression und -lokalisation wurde ein STED-Mikroskop (TCS SP8 STED, HC PL APO CS2 100x/1.4 Öl-Objektiv, Leica Microsystems) inklusive der zugehörigen Software (Application Suite X, Leica Microsystems) verwendet. Die Aufnahmen wurden im LIFDateiformat gespeichert, wobei die Pixelgröße auf 113,64 nm x 113,64 nm bzw. bei fünffacher Vergrößerung auf 22,73 nm x 22,73 nm festgelegt wurde. Weitere Einstellungen: pixel dwell time $400 \mathrm{~ns}$, scanning speed $600 \mathrm{~Hz}$, line averaging 32x. Die Lochblendenöffnung von 1 AUn resultierte in einer optischen Scheibendicke von $896 \mathrm{~nm}$. Jede Zelle wurde zunächst zur Beurteilung der Qualität durchlichtmikroskopisch aufgenommen. Danach folgten konfokale Übersichtsaufnahmen der Größe 116,25 nm x 116,25 nm und schließlich mittels 5x optischen Zooms vergrößerte STED-Aufnahmen der Größe 23,25 nm x 23,25 nm aus dem Bereich des TAT-Netzwerks.

STED-Laser der Wellenlänge $592 \mathrm{~nm}$ und $775 \mathrm{~nm}$ wurden mit einer Leistung von $20 \%$ bzw. $50 \%$ betrieben. Die Sekundärantikörper STAR 635P wurden mit Licht der Wellenlänge $635 \mathrm{~nm}$ (Weißlichtlaser) angeregt und die Fluoreszenz im Bereich von 650-700 nm detektiert. Entsprechend wurden STAR-488-Antikörper bei $500 \mathrm{~nm}$ angeregt und die Fluoreszenz im Bereich von 515-560 nm detektiert. Für maximale Auflösung wurde die STED-Laserleistung pro Färbeprotokoll angepasst. Für die Bearbeitung und Analyse der Rohaufnahmen wurde Fiji verwendet.

\subsubsection{Immunfluoreszenzfärbung fixierter Kardiomyozyten}

Für Immunfluoreszenzaufnahmen am STED-Mikroskop wurden AM und VM direkt nach der Isolation (siehe 2.5) fixiert. Hierzu wurden zunächst Deckgläser (Ø $18 \mathrm{~mm}$ ) mit $2 \mu \mathrm{l}$ Laminin $(2 \mathrm{mg} / \mathrm{ml})$ beschichtet und in eine Multivell-Zellkulturplatte gelegt. Von den in Perfusionspuffer gelösten Kardiomyozyten wurden 150 - $200 \mu \mathrm{l}$ auf jedem Deckglas platziert und dort 15 Minuten zum Absetzen belassen. Nach einem Waschschritt mit Perfusionspuffer wurden die verbleibenden Zellen mit $4 \%$ Paraformaldehyd (PFA) in PBS über 
fünf Minuten bei Raumtemperatur fixiert und anschließend einmalig mit PBS gewaschen. Um die Zellen zu permeabilisieren und unspezifische Proteinbindungsstellen zu blocken, wurden die Deckgläser bei $4{ }^{\circ} \mathrm{C}$ über Nacht mit Blockpuffer bedeckt, bestehend aus 0,2 \% Triton X-100 (Sigma-Aldrich, T9284) sowie $10 \%$ BCS (Thermo Fisher Scientific, SH30073) in PBS.

Am darauffolgenden Tag wurden die Zellen mit den in Blockpuffer verdünnten (1:500) Primärantikörpern behandelt und über Nacht bei $4{ }^{\circ} \mathrm{C}$ inkubiert. Nach drei Waschschritten mit Blockpuffer wurden am Folgetag die ebenfalls in Blockpuffer verdünnten (1:1.000) Sekundärantikörper auf die Deckgläser gegeben und drei Stunden bei Raumtemperatur im Dunkeln belassen. Drei weiteren Waschschritten mit PBS folgte die Einbettung der Deckgläser auf Objektträgern mittels Einbettmedium (ProLong ${ }^{\circledR}$ Gold Antifade mit DAPI, Invitrogen, P36934). Nach der Aushärtung des Einbettmediums über Nacht bei Raumtemperatur wurden die Präparate mit farblosem Nagellack versiegelt. Die verwendeten Primär- und Sekundärantikörper sind im Anhang (siehe 6.4.3 und 6.4.4) aufgelistet.

Für 3D-Rekonstruktionen konfokaler Z-Stacks (siehe Abbildung 13B) wurde in Fiji zunächst das Signal binarisiert. Mittels des Plugins 3D-Viewer wurde aus den Binärbildern ein 3D-Modell der jeweiligen Kardiomyozyte erstellt. Gelbe Färbung repräsentiert hier die überlappenden Signale von RyR2- (rot) und JPH2- bzw. RyR2-pS2808-Antikörpern (grün). Das unspezifische nukleäre RyR2-pS2808-Antikörper-Signal (Kreuzreaktion) wurde genutzt, um den Zellkern in blau darzustellen.

\subsubsection{Humane myokardiale Gewebeproben}

Humane linksatriale Gewebeproben für Immunfluoreszensaufnahmen am konfokalen Mikroskop wurden Patienten während der Kanülierung bei einer koronaren Bypass-Operation am offenen Herzen entnommen. Alle Patienten hatten der Prozedur zuvor schriftlich zugestimmt. Das Versuchsvorhaben wurde von der Ethikkommission der UMG geprüft und genehmigt (Nr. 21/10/00). Die Gewebeproben wurden über Nacht fixiert (Roti®-Histofix $4 \%$ ), in Paraffin eingebettet und in $4 \mu \mathrm{m}$ dicke Scheiben geschnitten. Die Schnitte wurden entparaffinisiert, rehydriert und zur Antigendemaskierung mit $10 \mathrm{mM}$ Natriumzitrat-Puffer behandelt. Die histologische Verarbeitung der Proben wurde von Birgit Schumann durchgeführt. Um die Proben zu permeabilisieren und unspezifische Proteinbindungsstellen zu blocken, wurden sie für eine Stunde mit Blockpuffer bedeckt, bestehend aus 0,1\% Triton X-100 sowie $4 \%$ bovinem Serumalbumin (BSA, Sigma-Aldrich, A8806) in PBS. Die Primärantikörper (rabbit anti-JPH2 und mouse anti-Cav3, siehe 6.4.3 im Anhang) wurden hier im Verhältnis 1:250 in PBS (4\% BSA) verdünnt und über Nacht zur Inkubation auf den Proben belassen. Am Folgetag wurden die Proben nach drei Waschschritten zwei Stunden lang mit Sekundärantikörpern inkubiert (STAR 580 und STAR 635P, siehe 6.4.4, hier 1:300 verdünnt). Schließlich wurden die Präparate mit ProLong® Gold Antifade eingebettet. 


\subsection{Analyse von JPH2- und RyR2-Clustern anhand von STED- Aufnahmen fixierter Kardiomyozyten}

Quantitative Analysen subzellulärer JPH2- und RyR2-Cluster-Eigenschaften basierten auf STED-Immunfluoreszenzaufnahmen wie oben beschrieben (siehe 2.9). Das Signalmuster der Immunfluoreszenz von JPH2 bzw. RyR2 wurde mittels zwei verschiedener FijiBefehlsabfolgen segmentiert:

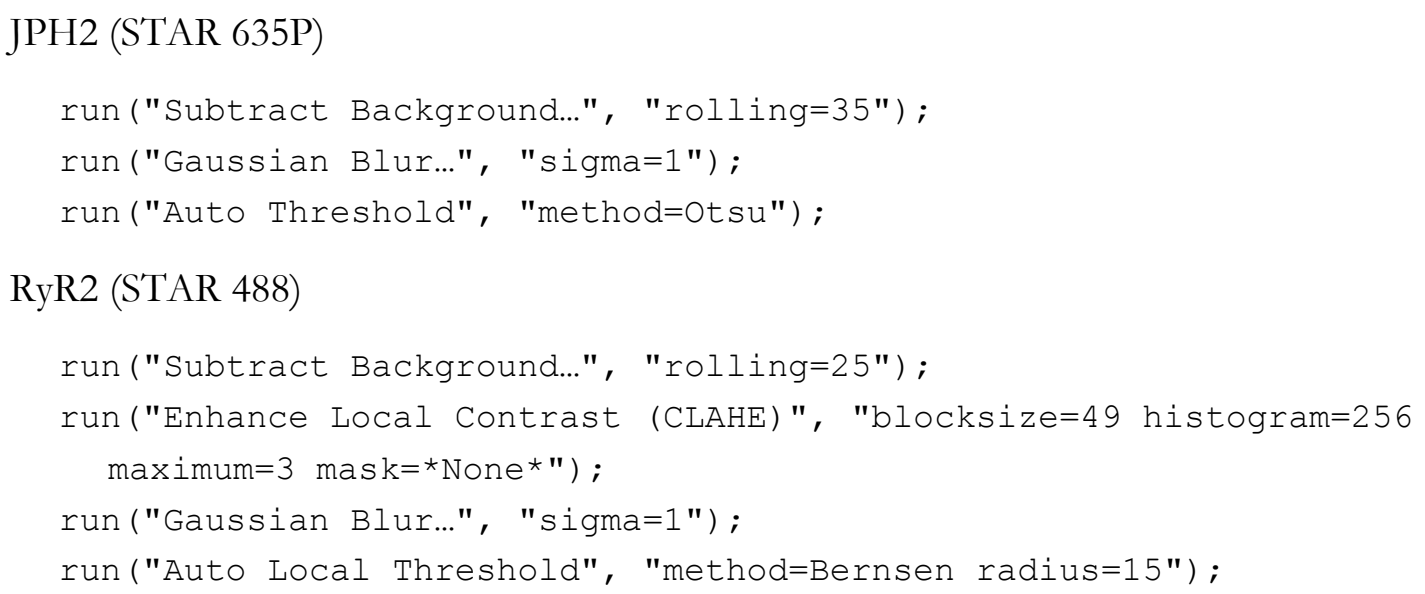

Sowohl für JPH2- als auch RyR2-Signale wurden Binärbilder verwendet, um den Flächenanteil des Signals (area fraction), die Dichte (density) und die Fläche (area) der Cluster zu berechnen. Für die Analyse der Überlappung (overlap) von JPH2- und RyR2-Clustern wurden neben vollständigen auch partielle Signalüberlagerungen akzeptiert. Die statistische Auswertung basierte auf individuellen Zelldaten.

\subsection{Elektrokardiographie}

Um auch etwaige Gruppenunterschiede der elektrischen Erregungsausbreitung und -rückbildung nach Tamoxifenbehandlung zu untersuchen, wurde einen Tag vor der Herzentnahme bei Versuchstieren beider Gruppen ein 6-Kanal-Elektrokardiogramm (EKG) geschrieben (IOX 2.10.0.40, emka TECHNOLOGIES), wobei sowohl Daten in Ruhe (BASAL, zehn Minuten) als auch unter $\beta$-adrenerger Stimulation (ISO, zehn Minuten) mittels i.p.-Injektion von Isoproterenol $(1 \mathrm{mg} / \mathrm{kg}$ Körpergewicht, Sigma-Aldrich, I5627) erhoben wurden.

Die Elektrokardiographie erfolgte in Narkose mit 1,5\% Isofluran in Sauerstoff $(1 \mathrm{l} / \mathrm{min})$ über eine Nasenmaske (MiniVent 845, Harvard Apparatus). Die Ableitung des Extremitäten-EKG erfolgte mittels speziell dimensionierter subkutaner EKG-Elektroden (Maus, emka TECHNOLOGIES), welche vorübergehend durch Haut-Klebeband stabilisiert wurden, um das Verletzungspotential durch Verrutschen der Elektroden sowie Störsignale durch Bewegungsartefakte und unnötige Versuchswiederholungen zu minimieren.

Die Auswertung der elektrokardiographischen Daten erfolgte sowohl manuell als auch semi-automatisch mittels ecgAUTO (Version 3.3.5.10, emka TECHNOLOGIES) und Excel 
(Microsoft Office 2016). Für die manuelle Analyse wurden pro Maus, Parameter (Herzfrequenz, P-Welle, PQ-Intervall, PR-Intervall) und Versuchsgruppe drei Messungen in Ableitung II durchgeführt und gemittelt. EKG-Spuren aus Ableitung I wurden verwendet, um die korrekte Erkennung des QRS-Komplexes zu verifizieren. Für eine zusätzliche Datenanalyse mit semi-automatischer Erkennung des QRS-Komplexes in Ableitung I wurde aus allen Aufnahmen beider Versuchsgruppen eine Referenzbibliothek individuell definierter Wellen manuell erstellt. EKG-Spuren aus Ableitung II wurden verwendet, um die korrekte Erkennung des QRS-Komplexes zu verifizieren. Die Daten wurden anschließend in Microsoft Excel importiert, auf das arithmetische Mittel der Herzfrequenz der Versuchstiere der Kontroll-Gruppe normalisiert (jeweils für BASAL und ISO) und zwischen Kontroll- und Knockdown-Gruppe verglichen. Repräsentative EKG-Spuren aus Ableitung I sind exemplarisch in Abbildung 20 gezeigt.

\subsection{Statistik}

Soweit nicht anders vermerkt, wurden alle Daten als Mittelwert \pm Standardfehler des arithmetischen Mittels angegeben. Die Unterschiede zwischen Kontroll- und KnockdownGruppe wurden in Excel mittels des heteroskedastischen t-Tests mit zweiseitiger Verteilung auf Signifikanz $(\mathrm{p}<0,05)$ getestet.

Die Daten des Verhältnisses von Herzgewicht zu Körpergewicht (siehe 3.3), der ClusterAnalyse von JPH2 und RyR2 (siehe 3.6.2) und der Elektrokardiographie (siehe 3.7) wurden in Box-Plots als Median, unteres und oberes Quartil dargestellt. Die Whisker repräsentieren jeweils den minimalen und maximalen Extremwert, der kurze horizontale Strich das arithmetische Mittel. 


\section{Ergebnisse}

Die Auswirkungen von JPH2-Expressionsveränderungen wurde bisher nicht in den funktionell bedeutungsvollen AM untersucht. Eine Vorläuferstudie hat mittels herzspezifischem JPH2-Knockdown ausschließlich Veränderungen in VM beschrieben (van Oort et al. 2011). Ziel war daher, den Effekt einer verminderten JPH2-Expression auf AM erstmals zu untersuchen. Es wurde von der Hypothese ausgegangen, dass verminderte JPH2-Expression mit atrialer Dysfunktion und globaler Herzinsuffizienz einhergeht.

\subsection{Proteinbiochemischer Nachweis der Expression von Junctophilin-2 im Atrium durch Western-Blot}

Auch im murinen Atrium wird JPH2 in substantieller Menge exprimiert. Wie in Abbildung 2 dargestellt, lag das Niveau der Proteinexpression von JPH2 in Wildtyp-Mäusen laut semiquantitativer Analyse im Western-Blot im Atrium allerdings bei nur $23 \pm 5 \%$ verglichen mit dem Ventrikel ( $p=0,001)$, während die Proteinexpression RyR2, dessen Funktion durch JPH2 stabilisiert wird (van Oort et al. 2011), nicht signifikant vermindert war $(84 \pm 8 \%$, $\mathrm{p}=0,14)$. Daraus ließ sich schließen, dass pro RyR2-Protomer verglichen mit dem Ventrikel im Atrium im Durchschnitt $28 \pm 6 \%$ JPH2-Einheiten stöchiometrisch im Verhältnis stehen können ( $\mathrm{p}=0,001 ; \mathrm{n}=6 / 6$ Herzen).

A

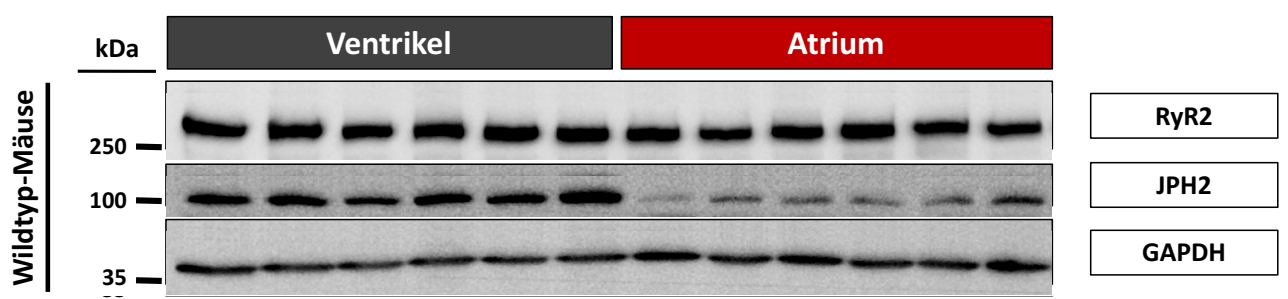

B

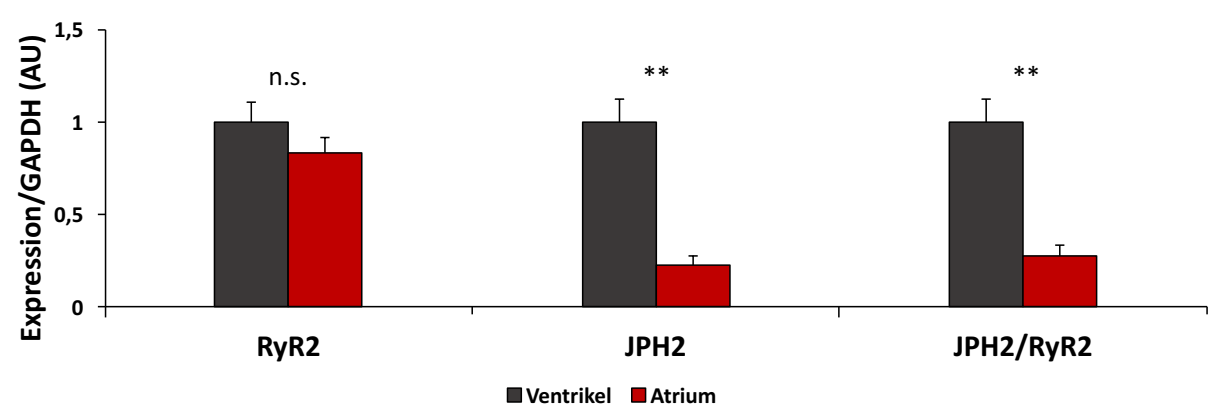

Abbildung 2: Nachweis der Proteinexpression von Junctophilin-2 im Ventrikel und Atrium muriner Wildtyp-Herzen.

(A) Western-Blots der Proteine Ryanodin-Rezeptor Typ 2 (RyR2) und Junctophilin-2 (JPH2) sowie Glyzerinaldehyd-3-phosphat-Dehydrogenase (GAPDH) als Referenzprotein; $n=6 / 6$ Herzen von Wildtyp-Mäusen. (B) Semiquantitative Analyse der Banden aus (A) belegt die Proteinexpression 
von JPH2 im Atrium in substantieller Menge, allerdings lediglich $23 \%$ des ventrikulären Vergleichswerts. Die atriale Proteinexpression von RyR2 unterschied sich nicht vom Ventrikel. Dadurch ergab sich atrial pro RyR2-Protomer eine um $72 \%$ geringere JPH2-Menge als im Ventrikel. Die Daten wurden pro Protein zunächst auf GAPDH und anschließend auf den Mittelwert der Ventrikel-Gruppe normalisiert (AU: arbitrary unit) und als Mittelwert \pm SEM dargestellt, $* *: \mathrm{p}<0,01$

\subsubsection{Knockdown von atrialem Junctophilin-2 zwei Wochen nach Tamoxifenbehandlung}

Zur Entwicklung eines im Gegensatz zur Studie von van Oort et al. (2011) weniger belastenden Induktionsprotokolls für den atrialen JPH2-Knockdown (Richtwert der Proteinmengenreduktion mindestens $50 \%$ ) wurden zunächst zwei unterschiedliche TamoxifenDosierungen und Behandlungszeiträume gewählt, um die Proteinreduktion und Überlebensrate der Versuchstiere zu optimieren: Nach einmaliger i.p.-Applikation von Tamoxifen mit entweder 40 oder $100 \mathrm{mg} / \mathrm{kg}$ Körpergewicht wurden jeweils zwei bzw. vier Wochen bis zur Herzentnahme abgewartet (Induktionsphase, siehe $2.1 \mathrm{im}$ Methodenteil). Die Ergebnisse der Behandlungsprotokolle „2p100mg“, „4p40mg“ und „2p40mg“ sind in Abbildung 3 dargestellt. In allen drei Versuchsgruppen zeigte sich eine signifikante Reduktion von JPH2 (siehe Tabelle 5 für Werte).

Während die Behandlung mit $40 \mathrm{mg} / \mathrm{kg}$ Körpergewicht Tamoxifen bei keinem Tier (weder nach zwei noch nach vier Wochen) letal war, überlebten in der 2p100mg-Gruppe nur $60 \%$ der Knockdown-Tiere die zwei Wochen dauernde Induktionsphase, also vergleichbar zur Studie von van Oort et al (2011). Um die Tiere möglichst wenig zu belasten, wurden daher alle weiteren Experimente nach dem 2p40mg-Schema durchgeführt, dementsprechend bezieht sich die Gruppenzuteilung „Knockdown“ nachfolgend auf ebendieses Protokoll.

Tabelle 5: Junctophilin-2-Knockdown im Atrium.

\begin{tabular}{llll}
\hline \hline \multicolumn{3}{c}{ Junctophilin-2-Proteinexpression (normalisiert) } & \\
\hline Behandlungsprotokoll & Kontrolle & Knockdown & p \\
\hline 1) $2 \mathrm{p} 100 \mathrm{mg}$ & $100 \pm 13 \%$ & $56 \pm 14 \%$ & 0,04 \\
2) $4 \mathrm{p} 40 \mathrm{mg}$ & $100 \pm 11 \%$ & $38 \pm 6 \%$ & 0,0009 \\
3) $2 \mathrm{p} 40 \mathrm{mg}$ & $100 \pm 11 \%$ & $42 \pm 6 \%$ & 0,002 \\
\hline
\end{tabular}

Mittelwert \pm SEM. Siehe Legende der Abbildung 3 für Abkürzungen und Erläuterungen. 


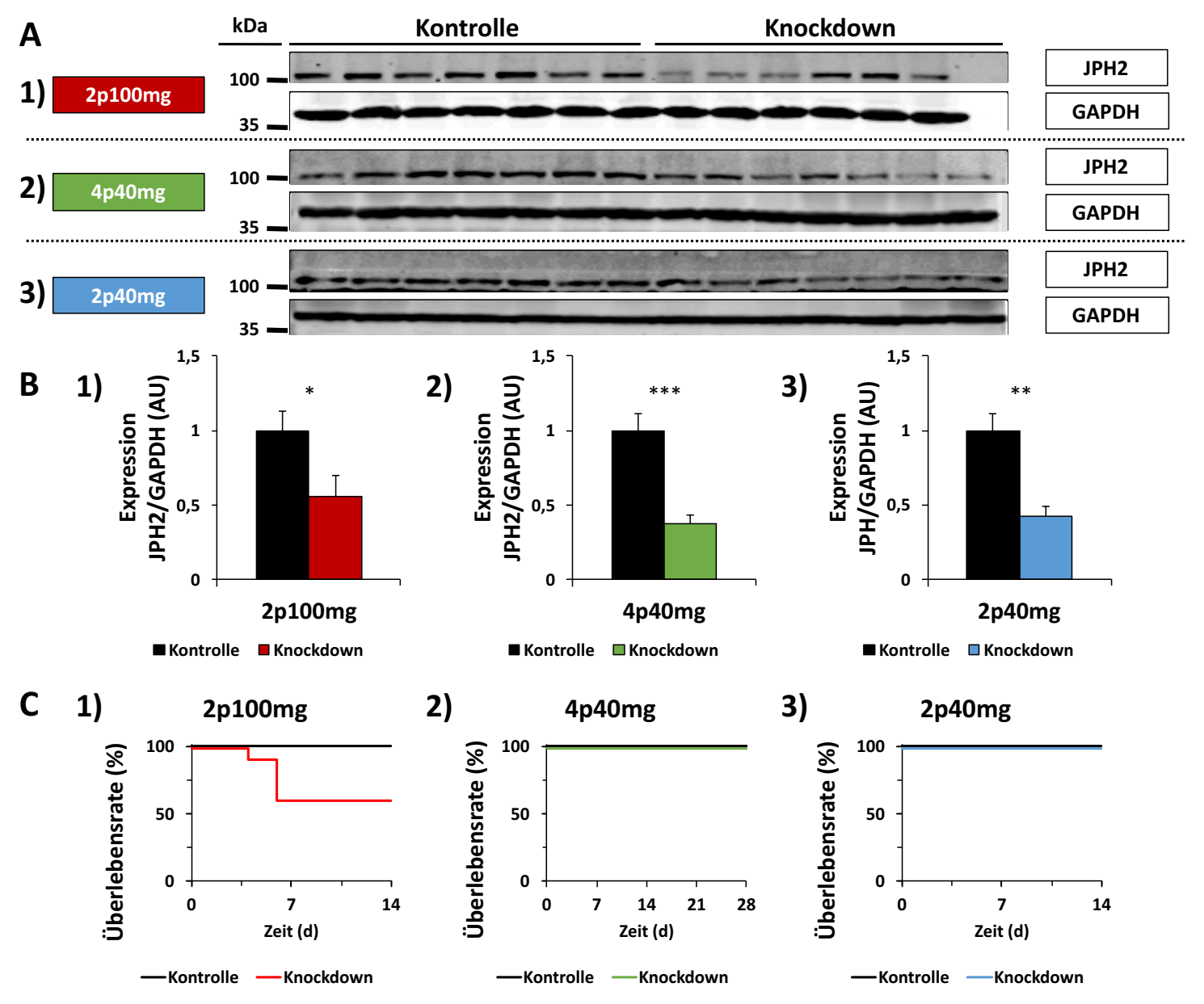

Abbildung 3: Junctophilin-2-Knockdown ohne Mortalität nach TamoxifenBehandlungsschema 2p40mg.

(A) Western-Blots der Proteine Junctophilin-2 (JPH2) und Glyzerinaldehyd-3-phosphatDehydrogenase (GAPDH) als Referenzprotein, aufgeteilt nach drei Induktionsprotokollen: 1) zwei Wochen Induktionsphase nach einmaliger Applikation von $100 \mathrm{mg} / \mathrm{kg}$ Tamoxifen (2p100mg) in rot, 2) vier Wochen nach $40 \mathrm{mg} / \mathrm{kg}$ Tamoxifen (4p40mg) in grün, und 3) zwei Wochen nach $40 \mathrm{mg} / \mathrm{kg}$ Tamoxifen (2p40mg) in blau; $\mathrm{n}=7 / 7$ Herzen pro Behandlungsprotokoll (außer 2p100mg: 7/6). (B) Semiquantitative Analyse der Banden aus (A) zeigt die Reduktion von JPH2 in allen Gruppen auf die Hälfte oder weniger im Vergleich mit der Kontroll-Gruppe. Die Daten wurden pro Protein zunächst auf GAPDH und anschließend auf den Mittelwert der Kontroll-Gruppe normalisiert (AU: arbitrary unit) und als Mittelwert \pm SEM dargestellt. *: $\mathrm{p}<0,05 ; * *: \mathrm{p}<0,01$; ***: $\mathrm{p}<0,001$. (C) Kaplan-Meier-Kurven der drei Induktionsprotokolle. In 2p100mg bestand bereits nach sechs Tagen eine Mortalität von $60 \%$ in der Knockdown-Gruppe, während in den anderen beiden Behandlungsgruppen alle Tiere die Induktionsphase überlebten.

\subsubsection{Junctophilin-2-Knockdown führt zu vermehrter SERCA2a- und Caveolin-3- Proteinexpression}

Nach erfolgter Behandlung nach obigem Schema 2p40mg wurden Western-Blots mit atrialem Gewebslysat der Gruppen Kontrolle und Knockdown durchgeführt (siehe Abbildung 4). Es wurden Antikörper gegen die Proteine RyR2, RyR2-pS2808, Cav1.2, SERCA2a, JPH2, 
PLN und Caveolin-3 (Cav3) verwendet und die Immunfluoreszenz semiquantitativ ausgewertet.

Neben der bereits beschriebenen Reduktion von JPH2 auf $42 \%$ wurden in der KnockdownGruppe verglichen mit der Kontroll-Gruppe signifikant höhere Werte für die Proteinexpression von SERCA2a und Cav3 festgestellt (siehe Tabelle 6). Die Proteinexpression von RyR2, RyR2-pS2808, Cav1.2 und PLN war nicht signifikant verändert. Setzte man die JPH2-Proteinexpression jeweils ins Verhältnis mit RyR2 und Cav1.2, ergab sich normalisiert zum Referenzprotein Glyzerinaldehyd-3-phosphat-Dehydrogenase (GAPDH) eine signifikant verminderte JPH2-Proteinexpression in der Knockdown-Gruppe. Außerdem lag stöchiometrisch im Verhältnis zu RyR2 eine signifikant reduzierte Menge des PKAphosphorylierten RyR2-pS2808 vor. Das Verhältnis der Proteinexpression von PLN und SERCA2a war nicht signifikant vermindert.

Tabelle 6: Werte der atrialen Proteinexpression nach Junctophilin-2-Knockdonn.

\begin{tabular}{lcc|l}
\hline \hline & Proteinexpression (normalisiert) & \\
\hline Protein & Kontrolle & Knockdown & p \\
\hline JPH2 & $100 \pm 11 \%$ & $42 \pm 6 \%$ & 0,002 \\
RyR2 & $100 \pm 9 \%$ & $91 \pm 6 \%$ & 0,45 \\
JPH2/RyR2 & $100 \pm 17 \%$ & $44 \pm 7 \%$ & 0,02 \\
RyR2-pS2808 & $100 \pm 15 \%$ & $71 \pm 6 \%$ & 0,13 \\
RyR2-pS2808/RyR2 & $100 \pm 5 \%$ & $76 \pm 6 \%$ & 0,02 \\
SERCA2a & $100 \pm 3 \%$ & $143 \pm 7 \%$ & 0,0009 \\
PLN & $100 \pm 13 \%$ & $102 \pm 18 \%$ & 0,94 \\
PLN/SERCA2a & $100 \pm 13 \%$ & $72 \pm 14 \%$ & 0,16 \\
Cav3 & $100 \pm 12 \%$ & $150 \pm 12 \%$ & 0,02 \\
Cav1.2 & $100 \pm 10 \%$ & $74 \pm 7 \%$ & 0,08 \\
JPH2/Cav1.2 & $100 \pm 6 \%$ & $57 \pm 9 \%$ & 0,002 \\
\hline \hline
\end{tabular}

Mittelwert \pm SEM. Siehe Legende der Abbildung 4 für Abkürzungen und Erläuterungen. 
A

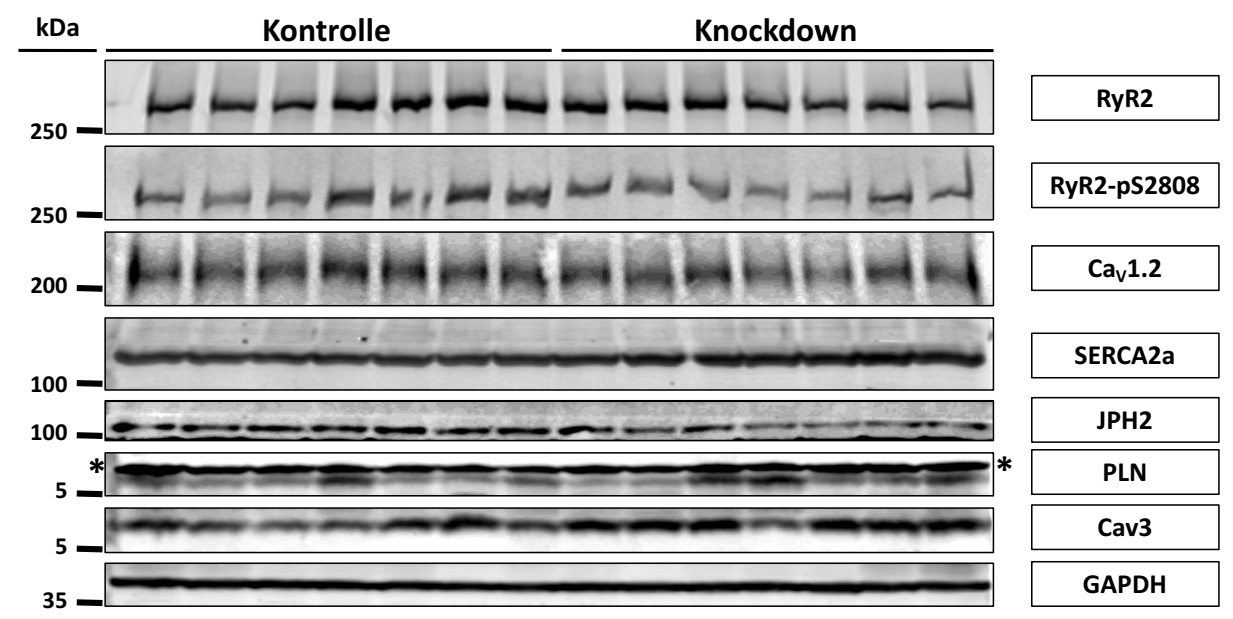

B

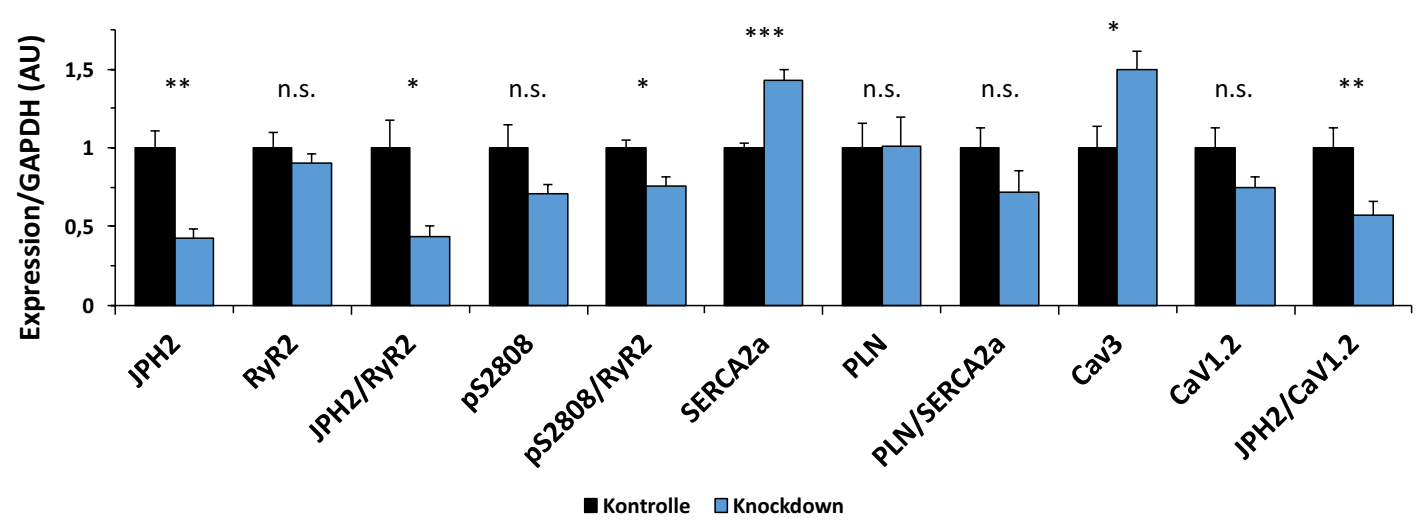

Abbildung 4: Junctophilin-2-Knockdown führt zu atrialen Expressionsveränderungen kardial bedeutsamer Proteine.

Western-Blots der Proteine Ryanodin-Rezeptor Typ 2 (RyR2), PKA-abhängig Ser2808phosphorylierter RyR2 (RyR2-pS2808), spannungsabhängiger L-Typ-Ca ${ }^{2+}-$ Kanal 1.2 (Cav1.2), $\mathrm{Ca}^{2+}$ ATPase des sarkoendoplasmatischen Retikulums 2a (SERCA2a), Junctophilin-2 (JPH2), Phospholamban (PLN; *: unspezifische Bande über der PLN-Bande) und Caveolin-3 (Cav3) sowie Glyzerinaldehyd-3-phosphat-Dehydrogenase (GAPDH) als Referenzprotein; $n=7 / 7$ Herzen. (B) Semiquantitative Analyse der Banden aus (A) zeigt den erfolgreichen Knockdown von Junctophilin-2 auf $42 \%$ verglichen mit der Kontroll-Gruppe. Die Proteinexpression von SERCA2a und Cav3 stieg um $43 \%$ bzw. 50 \%. Das Verhältnis von JPH2 zu RyR2 und Cav1.2 war auf 44 \% bzw. 57 \% vermindert. Die Daten wurden pro Protein zunächst auf GAPDH und anschließend auf den Mittelwert der Kontroll-Gruppe normalisiert (AU: arbitrary unit) und als Mittelwert \pm SEM dargestellt. $*: \mathrm{p}<0,05 ; * *: \mathrm{p}<0,01 ; * * *$ : $<0,001 ;$ n.s.: nicht signifikant.

\subsection{Reduzierte linkskardiale Kontraktilität ohne Hypertrophiezeichen nach Junctophilin-2-Knockdown}

Um die Auswirkungen des Tamoxifen-induzierten JPH2-Knockdowns auf strukturelle und funktionelle Herzparameter in vivo zu untersuchen, wurden als Blindversuch zu zwei Zeitpunkten echokardiographische Daten unter Isoflurannarkose (1,5 Vol.-\%) erhoben: Einen Tag vor der Tamoxifen-Applikation zur Kontrolle (PRE TAM) und zwei Wochen später, 
einen Tag vor der Herzentnahme (POST TAM $=2 \mathrm{p} 40 \mathrm{mg}$ ). Es wurden atriale und ventrikuläre Parameter vermessen (linksatrialer Durchmesser diastolisch, LAIDd; linksventrikulärer Durchmesser diastolisch, LVIDd; linksventrikuläre Vorderwanddicke diastolisch, AWThd; siehe 2.3).

Abbildung 5 und Tabelle 7 zeigen die gewonnen Ergebnisse. Vor der Tamoxifenbehandlung bestanden weder strukturelle noch funktionelle Unterschiede zwischen der Kontroll- und Knockdown-Gruppe. Die strukturellen Parameter (LAIDd, LVIDd und AWThd) blieben auch nach der Intervention in beiden Gruppen statistisch unverändert.

Tabelle 7: Messwerte der Echokardiographie.

\begin{tabular}{l|ll|l|ll|l}
\hline & \multicolumn{3}{|c|}{ PRE TAM } & \multicolumn{3}{c}{ POST TAM } \\
\hline Parameter & Kontrolle & Knockdown & p & Kontrolle & Knockdown & p \\
\hline LAIDd (mm) & $1,92 \pm 0,09$ & $2,12 \pm 0,10$ & 0,15 & $1,91 \pm 0,07$ & $2,01 \pm 0,07$ & 0,30 \\
LVIDd (mm) & $4,32 \pm 0,05$ & $4,46 \pm 0,07$ & 0,13 & $4,37 \pm 0,06$ & $4,51 \pm 0,07$ & 0,13 \\
AWThd (mm) & $0,74 \pm 0,01$ & $0,74 \pm 0,02$ & 0,99 & $0,73 \pm 0,02$ & $0,76 \pm 0,02$ & 0,18 \\
\hline LAFS (\%) & $15,63 \pm 1,31$ & $15,90 \pm 1,14$ & 0,88 & $16,54 \pm 0,91$ & $12,93 \pm 0,89$ & 0,008 \\
LVEF (\%) & $42,63 \pm 1,10$ & $40,18 \pm 1,27$ & 0,15 & $39,47 \pm 1,20$ & $31,81 \pm 1,58$ & 0,0004 \\
LVFS (\%) & $21,20 \pm 0,80$ & $19,10 \pm 0,84$ & 0,07 & $18,86 \pm 0,70$ & $14,92 \pm 1,0$ & 0,002 \\
\hline \hline
\end{tabular}

Mittelwert \pm SEM. Siehe Legende der Abbildung 5 für Abkürzungen und Erläuterungen.

Funktionell bildete sich allerdings in der Knockdown-Gruppe eine Störung der atrialen Kontraktilität aus: Die linksatriale Verkürzungsfraktion (LAFS) war - trotz hochregulierter SERCA2-Proteinexpression (siehe 3.1.2) - mit 12,9\% signifikant geringer als in der Kontroll-Gruppe. Die Verminderung der linksventrikulären Ejektionsfraktion (LVEF) und Verkürzungsfraktion (LVFS) auf 31,8\% bzw. auf 14,9\% zeigte eine linksventrikuläre Herzinsuffizienz an. 
A
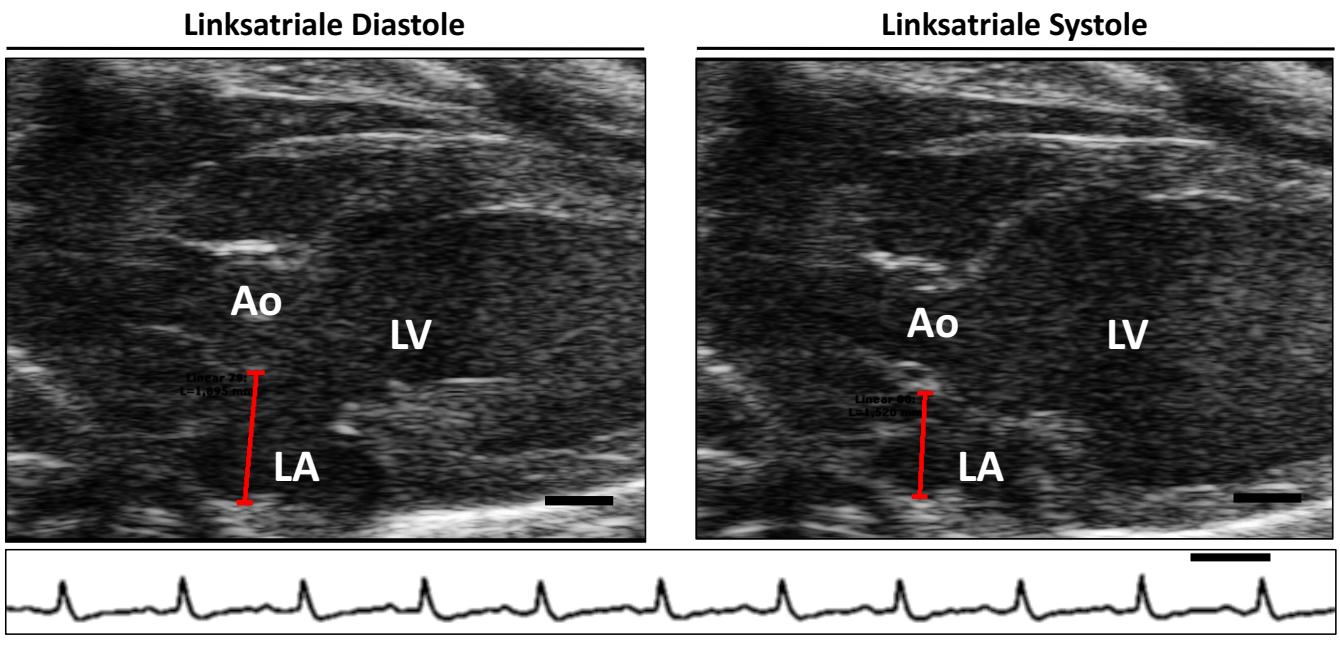

B
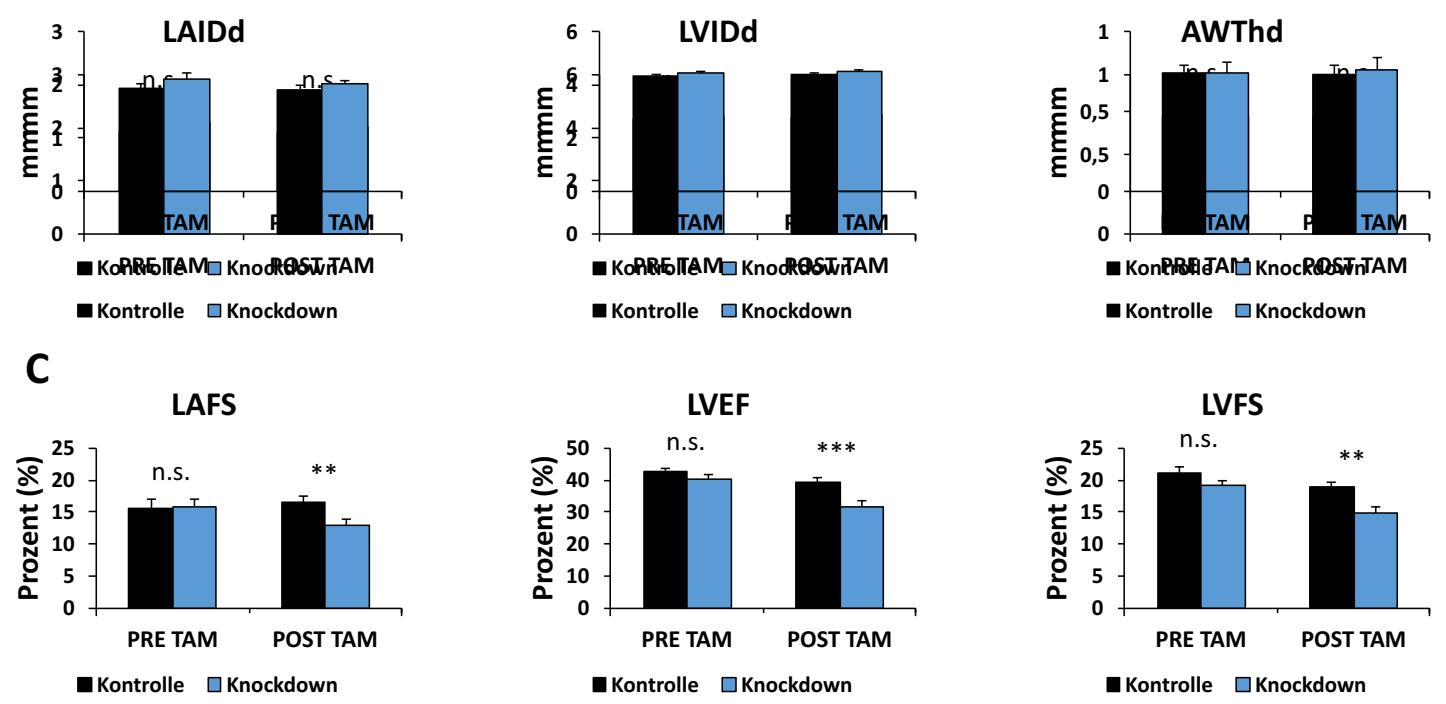

Abbildung 5: Junctophilin-2-Knockdown induziert Verschlechterung funktioneller Herzparameter.

(A) Die oberen Bilder zeigen Beispiele transthorakaler Echokardiographie einer Maus der KontrollGruppe (lange Achse parasternal) mit Ausmessung des linksatrialen Innendurchmessers in der Diastole (LAIDd) und in der Systole (LAIDs), in rot markiert. Ao: Aortenwurzel; LV: linker Ventrikel; LA: linkes Atrium; Maßstab: $1 \mathrm{~mm}$. Das untere Bild zeigt die EKG-Spur während der Echokardiographie-Messungen. Maßstab: 100 ms. (B) Darstellung echokardiographischer Daten jeweils vor (PRE TAM) und zwei Wochen nach (POST TAM) der Behandlung mit $40 \mathrm{mg}$ Tamoxifen. Normale strukturelle Herzparameter PRE und POST TAM in beiden Gruppen; $n=16 / 18$ Mäuse. (C) POST TAM Abnahme der funktionellen Parameter LAFS, LVEF und LVFS in der KnockdownGruppe. Darstellung der Daten in (B) und (C) als Mittelwert \pm SEM; **: $\mathrm{p}<0,01 ;{ }^{* * *}: \mathrm{p}<0,001$; n.s.: nicht signifikant.

\subsection{Erhöhtes Herzgewicht nach Junctophilin-2-Knockdown}

Das Herz- und Körpergewicht der Versuchstiere wurde nach Herzentnahme manuell bestimmt. Während sich das Körpergewicht nicht unterschied, zeigte sich ein signifikanter 
Anstieg des Herzgewichts um 0,03 g und des Verhältnisses Herzgewicht zu Körpergewicht um 0,14\% in der Knockdown-Gruppe (siehe Abbildung 6 und Tabelle 8) bei echokardiographischem Ausschluss von linksventrikulärer Hypertrophie und Dilatation (siehe 3.2).
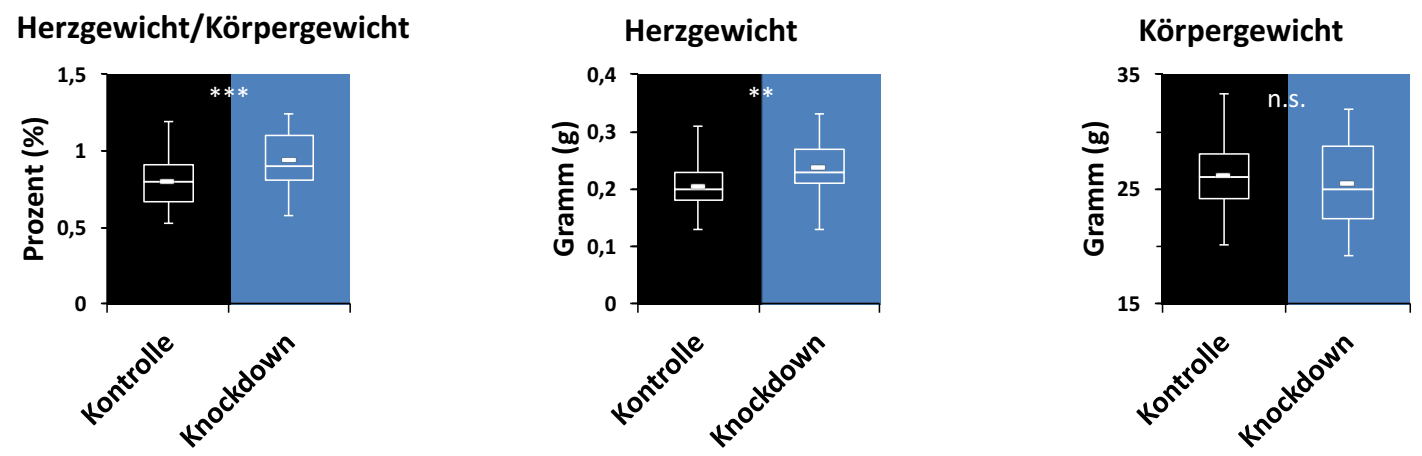

Abbildung 6: Herzgewicht-Zunahme in der Junctophilin-2-Knockdown-Gruppe.

Darstellung manuell erhobener Daten zum Herz- und Körpergewicht aller Versuchstiere des Behandlungsschemas 2p40mg. Durch das erhöhte Herzgewicht bei unverändertem Körpergewicht ergab sich in der Knockdown-Gruppe ein signifikanter Unterschied zur Kontroll-Gruppe auch im Verhältnis der beiden Parameter; $n=$ 41/43 Mäuse. Die Box-Plots repräsentieren den Median mit unterem und oberem Quartil, die Whisker das Minimum und Maximum, der kurze horizontale Strich das arithmetische Mittel. **: $\mathrm{p}<0,01 ;{ }^{* * *}$ : $<0,001 ;$ n.s.: nicht signifikant.

Tabelle 8: Messwerte des Herz- und Körpergewichts.

\begin{tabular}{lll|l}
\hline \hline \multicolumn{3}{c}{ 2p40mg } & \\
\hline Parameter & Kontrolle & Knockdown & $\mathbf{p}$ \\
\hline Herzgewicht/Körpergewicht (\%) & $0,79 \pm 0,03$ & $0,93 \pm 0,03$ & 0,0003 \\
Herzgewicht $(\mathrm{g})$ & $0,20 \pm 0,01$ & $0,23 \pm 0,01$ & 0,004 \\
Körpergewicht $(\mathrm{g})$ & $26,2 \pm 0,50$ & $25,4 \pm 0,56$ & 0,33 \\
\hline \hline
\end{tabular}

Mittelwert \pm SEM. Siehe Legende der Abbildung 6 für Erläuterungen.

\subsection{Konfokalmikroskopische Aufnahmen lebender Kardiomyozyten}

Aus früheren Studien ist bekannt, dass sich bei atrialer Hypertrophie (induziert durch TAC) am TAT-Netzwerk der Myozyten teilweise gravierende Umbauvorgänge ergeben (Brandenburg et al. 2016a). Junctophilin-2 stabilisiert als Strukturprotein im Ventrikel den JMC, also die Verbindung zwischen Komponenten des TAT-Netzwerks und der Membran des SR (van Oort et al. 2011). Da auch im Atrium die Expression von JPH2 nachgewiesen wurde (siehe 3.1), waren Umbauprozesse des atrialen TAT-Netzwerks nach JPH2Knockdown von besonderem Interesse.

Lebende AM (2p40mg) wurden nach der Isolation (siehe 2.5) mit dem lipophilen Farbstoff di-8-ANEPPS gefärbt und mittels konfokaler Laser-scanning-Mikroskopie visualisiert (siehe 2.6), um spezifische Membrankomponenten des TAT-Netzwerks analysieren zu können. 
Pro Zelle wurde sowohl eine Durchlichtaufnahme als auch eine Aufnahme des di-8ANEPPS-Signals angefertigt.

\subsubsection{Keine atriale Zellhypertrophie nach Junctophilin-2-Knockdown}

Anhand der Durchlichtaufnahmen einer größeren Stichprobe ( $n=54 / 56$ Zellen aus jeweils drei Mäusen pro Gruppe) wurden sowohl die Zelllänge und -breite gemessen als auch das Verhältnis von Breite zu Länge berechnet (siehe Abbildung 7). Bei keinem der genannten Parameter zeigte sich ein signifikanter Unterschied zwischen der Kontroll- und der Knockdown-Gruppe (siehe Tabelle 9). Somit hatte die 2p40mg-Behandlung keine Zellhypertrophie zur Folge.

Tabelle 9: Vermessung lebender atrialer Myozyten.

\begin{tabular}{lll|l}
\hline \multicolumn{4}{c}{ 2p40mg } \\
\hline Parameter & Kontrolle & Knockdown & $\mathbf{p}$ \\
\hline Länge $(\mu \mathrm{m})$ & $124,5 \pm 3,2$ & $126,9 \pm 4,0$ & 0,64 \\
Breite $(\mu \mathrm{m})$ & $16,2 \pm 0,4$ & $16,5 \pm 0,5$ & 0,73 \\
Breite/Länge $(\%)$ & $13,7 \pm 0,5$ & $13,7 \pm 0,7$ & 0,80 \\
\hline \hline
\end{tabular}

Mittelwert \pm SEM. Siehe Legende der Abbildung 7 für Erläuterungen.

A

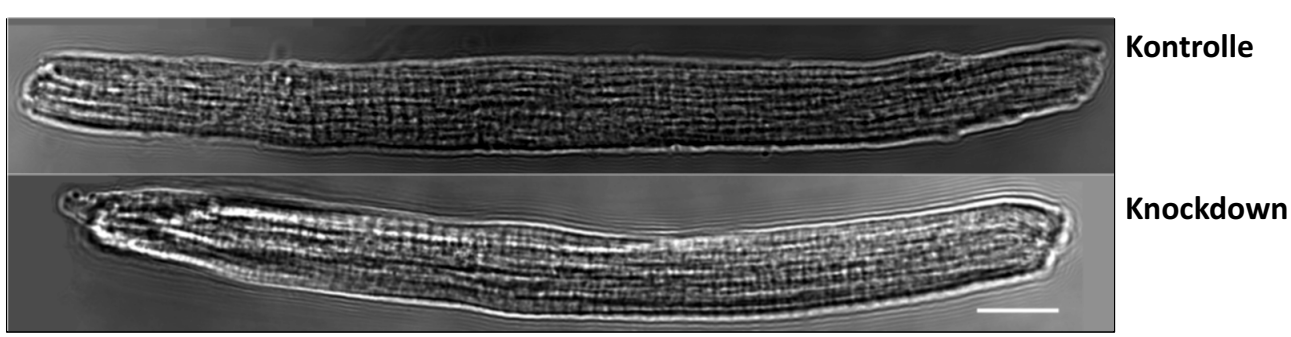

B
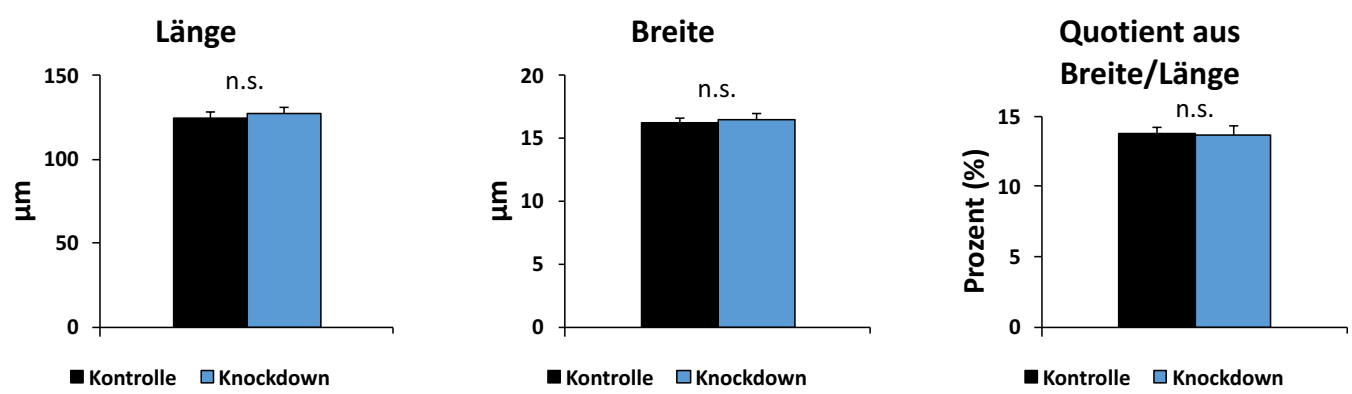

Abbildung 7: Junctophilin-2-Knockdown führt nicht zu Hypertrophie atrialer Kardiomyozyten.

(A) Repräsentative durchlichtmikroskopische Aufnahmen lebender atrialer Kardiomyozyten. Maßstab: $10 \mu \mathrm{m}$. (B) Messungen dieser Myozyten zeigten im Vergleich zwischen der Kontroll- und der Knockdown-Gruppe weder signifikante Veränderungen in Länge oder Breite, noch in deren Verhält- 
nis. Die Daten sind als Mittelwert \pm SEM bei $\mathrm{n}=54 / 56$ Zellen von $3 / 3$ Herzen präsentiert; n.s.: nicht signifikant.

\subsubsection{Junctophilin-2-Knockdown induziert eine Proliferation des TAT-Netzwerkes durch Zunahme axialer Tubuli}

Während VM ein regelhaft verzweigtes TAT-Netzwerk aus überwiegend T'T aufweisen, die im $90^{\circ}$-Winkel zur Längsachse der Zelle angeordnet und mit nur wenigen AT verbunden sind, verhält es sich bei AM laut Brandenburg et al. (2016a) genau umgekehrt: Der überwiegende Anteil der Tubuli ist ungefähr parallel zur Längsachse der Zelle, also axial orientiert; TT sind dagegen selten. Dabei ist die Dichte des TAT-Netzwerks bezogen auf die Zellfläche in AM geringer als in VM (Brandenburg et al. 2016a).

Mit dem Membranfarbstoff di-8-ANEPPS wurden Komponenten des TAT-Netzwerks lebender AM der Kontroll- und Knockdown-Gruppe sichtbar gemacht (siehe Abbildung 8) und analysiert (siehe 2.7.2). Hierfür wurden die Regionen der Oberflächenmembran und des Zellkerns ausgespart. Zunächst fällt das generelle Verteilungsmuster tubulärer Elemente in AM auf: Die zweigipfligen Histogramme veranschaulichen, dass auch Vorhofzellen AT (Gipfel bei $0^{\circ}$ ) und TT (Gipfel bei $90^{\circ}$ ) ausbilden, im Verhältnis jedoch deutlich mehr AT als TT. Die Subtraktion der Integrale voneinander (Knockdown - Kontrolle) ergab in der Knockdown-Gruppe einen erhöhten prozentualen Anteil von AT und einen verminderten Anteil von T'T am TAT-Netzwerk.

Diese Umverteilung wird in Abbildung 9 verdeutlicht: In der prozentualen Verteilung pro Zelle nahmen TT (Werte für $90 \pm 3^{\circ}$ aufsummiert) signifikant ab, während der Anteil von AT $\left(0 \pm 3^{\circ}\right)$ und OT $\left( \pm 45 \pm 3^{\circ}\right)$ gleich blieb (siehe Tabelle 10 für exakte Werte). Um dieser rein prozentualen Distribution auch absolute Werte entgegenzusetzen, wurde die jeweilige Länge des axialen, transversalen und obliquen Netzes (per Multiplikation des Prozentsatzes mit der Gesamtlänge des TAT-Netzwerks) ermittelt und zur Fläche des TAT-Netzwerks in Beziehung gesetzt. Als Ergebnis zeigte sich in der Knockdown-Gruppe eine signifikante Proliferation aller drei Tubulusausrichtungen, wobei besonders AT verglichen mit der Kontroll-Gruppe an Länge pro Netzwerkfläche zunahmen. Das Verhältnis von AT zu T'T war signifikant erhöht.

Teilt man die Gesamtlänge des TAT-Netzwerks (alle Winkelgrade eingeschlossen, also $0-179^{\circ}$ ) durch die Fläche, die es einnimmt, erhält man seine Dichte. In der KnockdownGruppe war diese signifikant um $0,12 \mu \mathrm{m} / \mu \mathrm{m}^{2}$ erhöht (siehe Abbildung 9).

Zusammengefasst konnte in der Knockdown-Gruppe im Vergleich zur Kontroll-Gruppe eine Umverteilung in der Orientierung tubulärer Elemente beobachtet werden: AT nahmen relativ gesehen zulasten von TT zu. Diesem Umstand lag allerdings kein eventuell anzunehmender Untergang transversaler Elemente zugrunde; vielmehr proliferierten alle Bestandteile des TAT-Netzwerks, allerdings war die Proliferation von AT am stärksten. Die Gesamtdichte des TAT-Netzwerks bezogen auf seine Fläche stieg ebenfalls signifikant. 
A

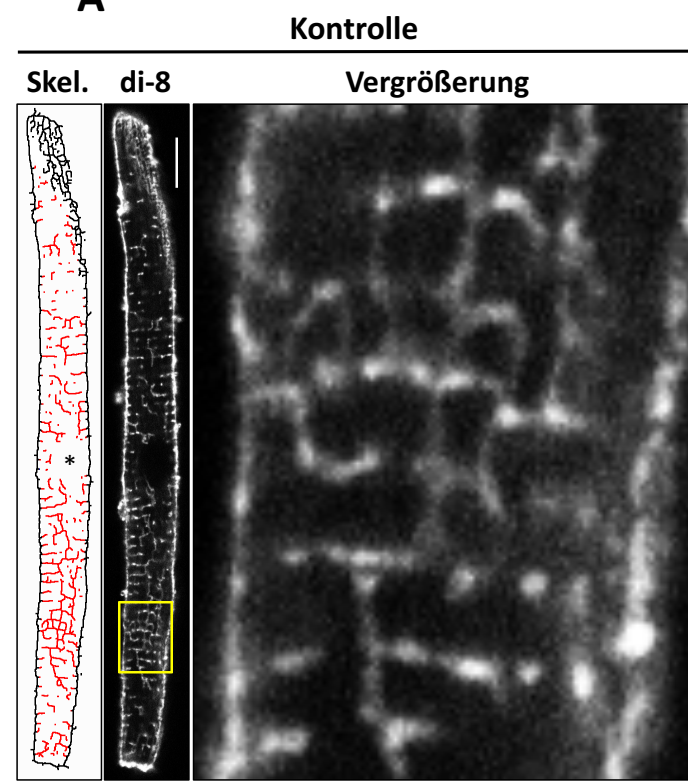

B
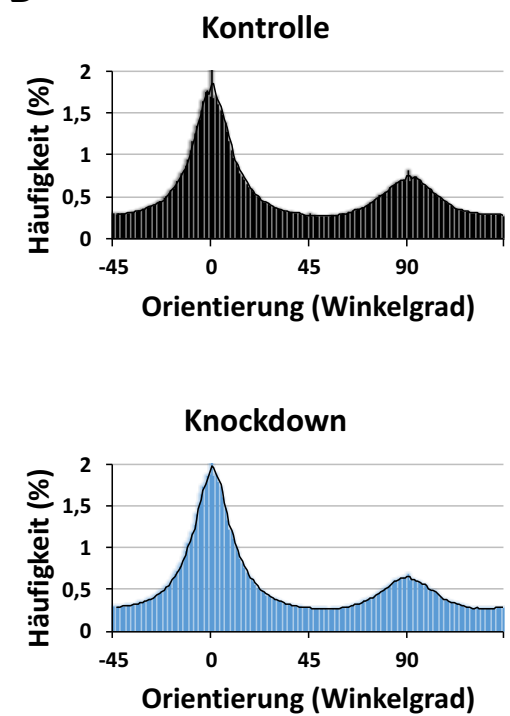

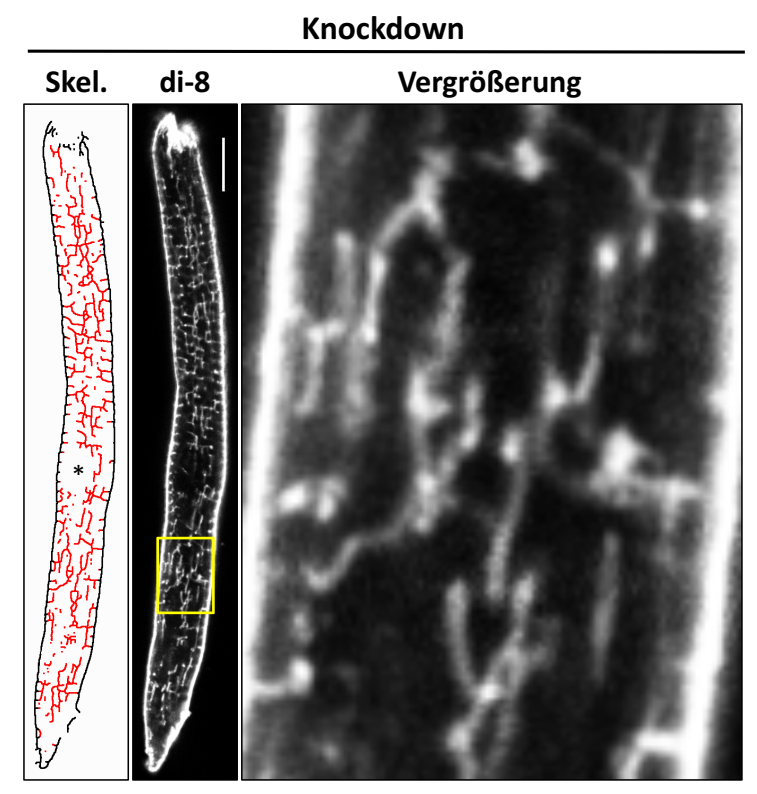

C
Differenz aus Knockdown minus Kontrolle

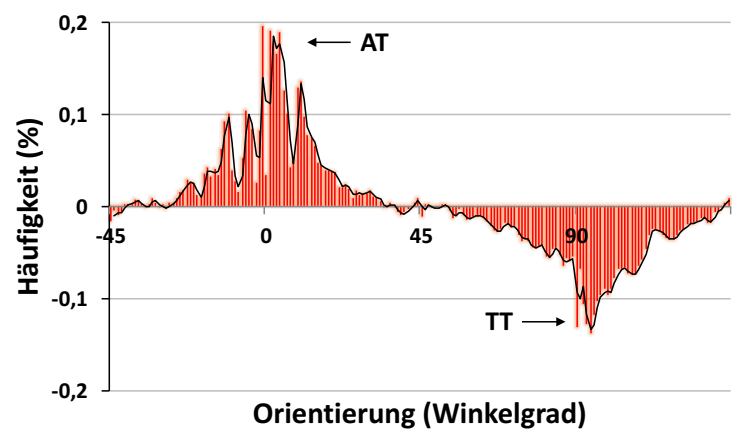

Abbildung 8: Verschiebung der Häufigkeiten der Tubulusausrichtungen nach Junctophilin-2-Knockdown zugunsten axialer Tubuli.

(A) Exemplarische konfokale Darstellung des TAT-Netzwerkes lebender atrialer Myozyten durch di-8-ANEPPS. Jeweils mittig ein Übersichtsbild der Zelle (di-8), rechts eine Vergrößerung (in di-8 mit gelber Box gekennzeichnet) und links in rot eine Darstellung des digitalisierten TATMembranskeletts (Skel.). Maßstab: $10 \mu \mathrm{m}$; *: Nukleus. (B) Histogramme der relativen Häufigkeit der Tubulusausrichtungen pro Zelle, jeweils für die Kontroll- und die Knockdown-Gruppe. (C) Darstellung der Subtraktion des Integrals des Kontroll-Histogramms vom Integral des KnockdownHistogramms. Darstellung der Daten aus (B) und (C) als Mittelwert bei $n=54 / 56$ Zellen von 3/3 Herzen. 
A

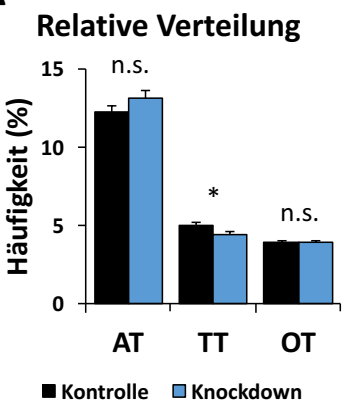

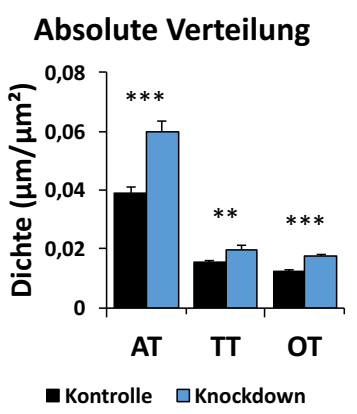

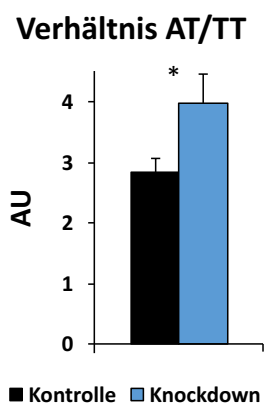

B

Dichte des TAT-Netzwerks

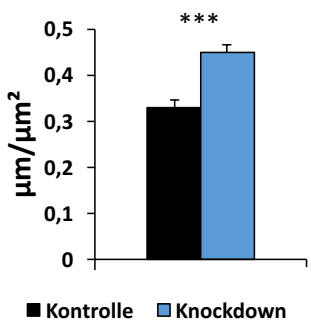

Abbildung 9: Proliferation des TAT-Netzwerks und der axialen Tubuli nach Junctophilin2-Knockdown.

(A) Darstellung der pro Zelle gemittelten Verteilung axialer (AT, $0 \pm 3^{\circ}$ ), transversaler (TT, $90 \pm 3^{\circ}$ ) und obliquer Tubuli (OT, $\pm 45 \pm 3^{\circ}$ ), jeweils für die Kontroll- und die KnockdownGruppe. Die relative Verteilung misst die Häufigkeit der Komponenten als prozentualen Anteil des TAT-Netzwerks. Die absolute Verteilung misst die Länge der jeweiligen Komponenten in $\mu \mathrm{m}$ bezogen auf die Fläche des TAT-Netzwerks in $\mu \mathrm{m}^{2}$. Axiale Tubuli proliferierten in der KnockdownGruppe stärker als transversale und oblique, während der Anteil der transversalen Tubuli relativ gesehen sogar sank. AU: arbitrary unit. (B) Darstellung der Dichte des TAT-Netzwerks, berechnet durch die Länge aller Tubuli in $\mu \mathrm{m}$ bezogen auf die Fläche des TAT-Netzwerks in $\mu \mathrm{m}^{2}$. In der Knockdown-Gruppe nahm die Dichte signifikant zu. Die Daten aus (A) und (B) werden als Mittelwert \pm SEM bei $\mathrm{n}=54 / 56$ Zellen von $3 / 3$ Herzen präsentiert. *: $\mathrm{p}<0,05$; ** $\mathrm{p}<0,01$; ***: $\mathrm{p}<0,001$; n.s.: nicht signifikant.

Tabelle 10: Ergebnisse der Orientierungs- und Dichteanalyse.

\begin{tabular}{l|lll|l}
\hline \hline \multicolumn{5}{c}{ 2p40mg } \\
\hline \multicolumn{1}{l|}{ Parameter } & Kontrolle & Knockdown & $\mathbf{p}$ \\
\hline \multirow{2}{*}{ Häufigkeit $(\%)$} & AT & $12,2 \pm 0,4$ & $13,1 \pm 0,6$ & 0,20 \\
& TT & $5,0 \pm 0,2$ & $4,4 \pm 0,2$ & 0,04 \\
& OT & $3,9 \pm 0,1$ & $3,9 \pm 0,1$ & 0,86 \\
\hline \multirow{2}{*}{ Dichte $\left(\mu \mathrm{m} / \mu \mathrm{m}^{2}\right)$} & AT & $0,039 \pm 0,002$ & $0,060 \pm 0,004$ & 0,000002 \\
& TT & $0,015 \pm 0,001$ & $0,020 \pm 0,001$ & 0,003 \\
\hline Verhältnis AT/TT $(\mathrm{AU})$ & OT & $0,012 \pm 0,001$ & $0,017 \pm 0,001$ & 0,00000001 \\
\hline Dichte TAT-Netzwerk $\left(\mu \mathrm{m} / \mu \mathrm{m}^{2}\right)$ & $0,33 \pm 0,02$ & $0,45 \pm 0,02$ & 0,03 \\
\hline \hline
\end{tabular}

Mittelwert \pm SEM. Siehe Legende der Abbildung 9 für Abkürzungen und Erläuterungen. 


\subsection{Histomorphologie nach Junctophilin-2-Knockdown}

Zum Ausschluss von Hypertrophie und Fibrose als unspezifische Ursachen der oben echokardiographisch beschriebenen kardialen Dysfunktion nach JPH2-Knockdown (siehe 3.2) wurden histologische Schnitte angefertigt.

Jeweils drei Herzen der Kontroll- und 2p40mg Knockdown-Gruppe wurden wie in 2.2 beschrieben für histologische Untersuchungen entnommen, jedoch nicht gespült, sondern umgehend fixiert und später am Mikrotom in $4 \mu \mathrm{m}$ dicke Scheiben geschnitten. Siehe Kapitel 2.8 für die genaue Aufarbeitung der Schnitte.

\subsubsection{Hämatoxylin-Eosin-Färbung zum Ausschluss von Hypertrophie}

Zur Beurteilung der Probenqualität, Gewebsarchitektur und Zellmorphologie wurden histologische Anschnitte, die jeweils beide Atrien, die Aorta und den rechten Ventrikel darstellten, dem konventionellen HE-Färbeprotokoll unterzogen. Neben einem Übersichtsbild wurden 40-fach vergrößerte Aufnahmen des interatrialen Septums sowie des atrialen Interstitiums, Epi- und Endokards angefertigt.

Im Übersichtsbild (siehe Abbildung 10) stellen sich die Atrien sowohl in der Kontroll- als auch der Knockdown-Gruppe morphologisch vergleichbar dar. Der Durchmesser war unverändert, es ließen sich weder Dilatation noch Gewebshypertrophie beobachten. Aus den vergrößerten Aufnahmen wird ersichtlich, dass die transversal und axial angeschnittenen AM auch nach JPH2-Knockdown nicht auffällig verändert waren: gleich große Zellkerne und Zellen, normales Verhältnis von Zellkern zu Zellfläche, keine zelluläre Hypertrophie, keine eingewanderten Zelltypen. Endokard und Epikard stellten sich schlank dar und lagen dem Myokard direkt an. Zusätzliches Fett oder Fibrose war nicht erkennbar. Diese Daten bestätigen obige Ergebnisse der Zellisolation in Bezug auf fehlende atriale Zellhypertrophie nach JPH2-Knockdown (siehe 3.4.1). 


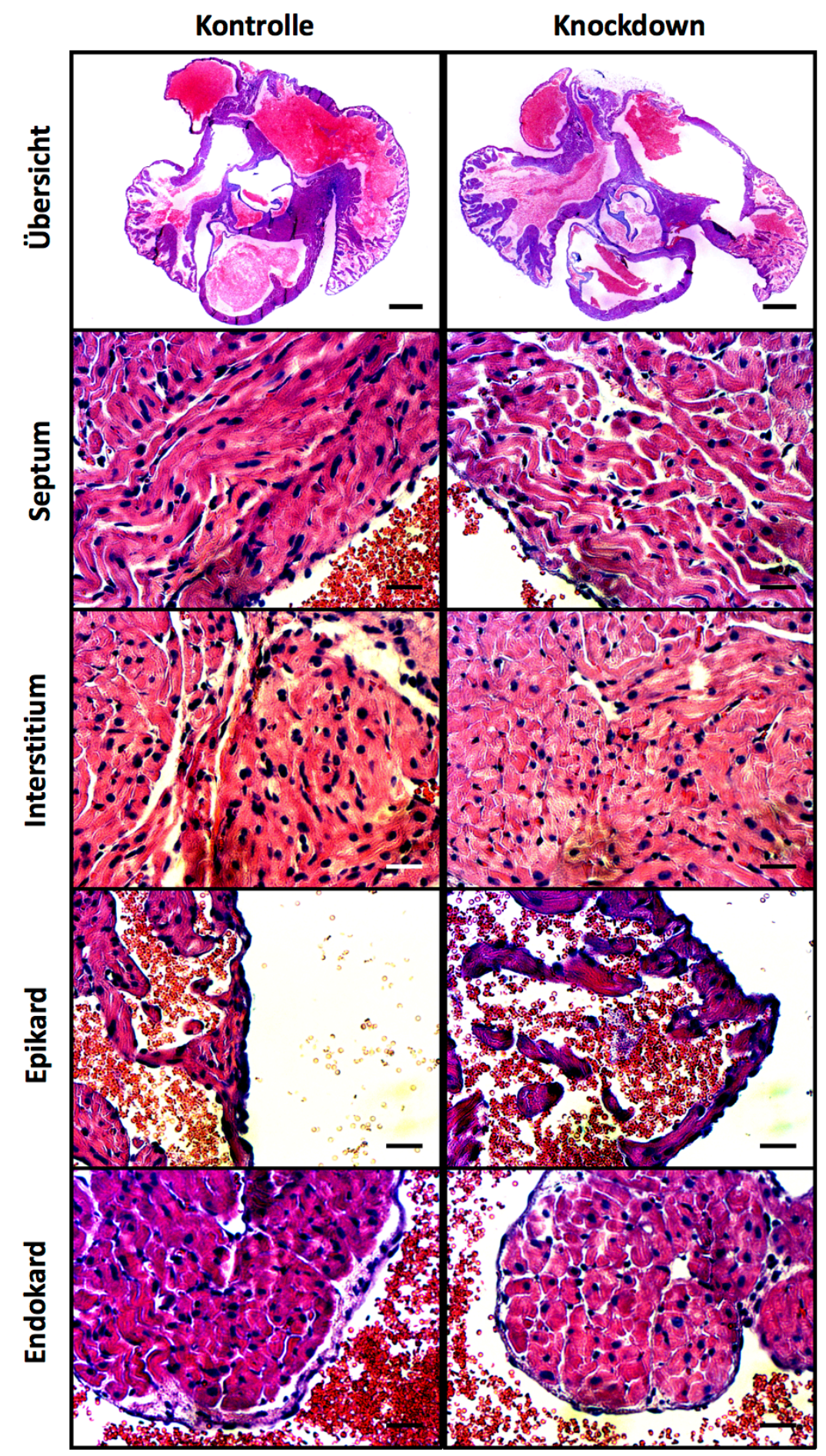

Abbildung 10: Hämatoxylin-Eosin-Färbung schließt Hypertrophie nach Junctophilin-2Knockdown aus.

Repräsentative Darstellung HE-gefärbter histologischer Schnitte. Jeweils ein Übersichtsbild (Anschnitte beider Atrien, der Aorta und des rechten Ventrikels) und vier vergrößerte Aufnahmen (40x) verschiedener Lokalisationen der Herzvorhöfe. Maßstäbe: Übersicht $500 \mu \mathrm{m}$, ansonsten $50 \mu \mathrm{m} ; \mathrm{n}=3 / 3$ Herzen. 


\subsubsection{Sirius-Red/Fast-Green-Färbung zum Ausschluss von Fibrose}

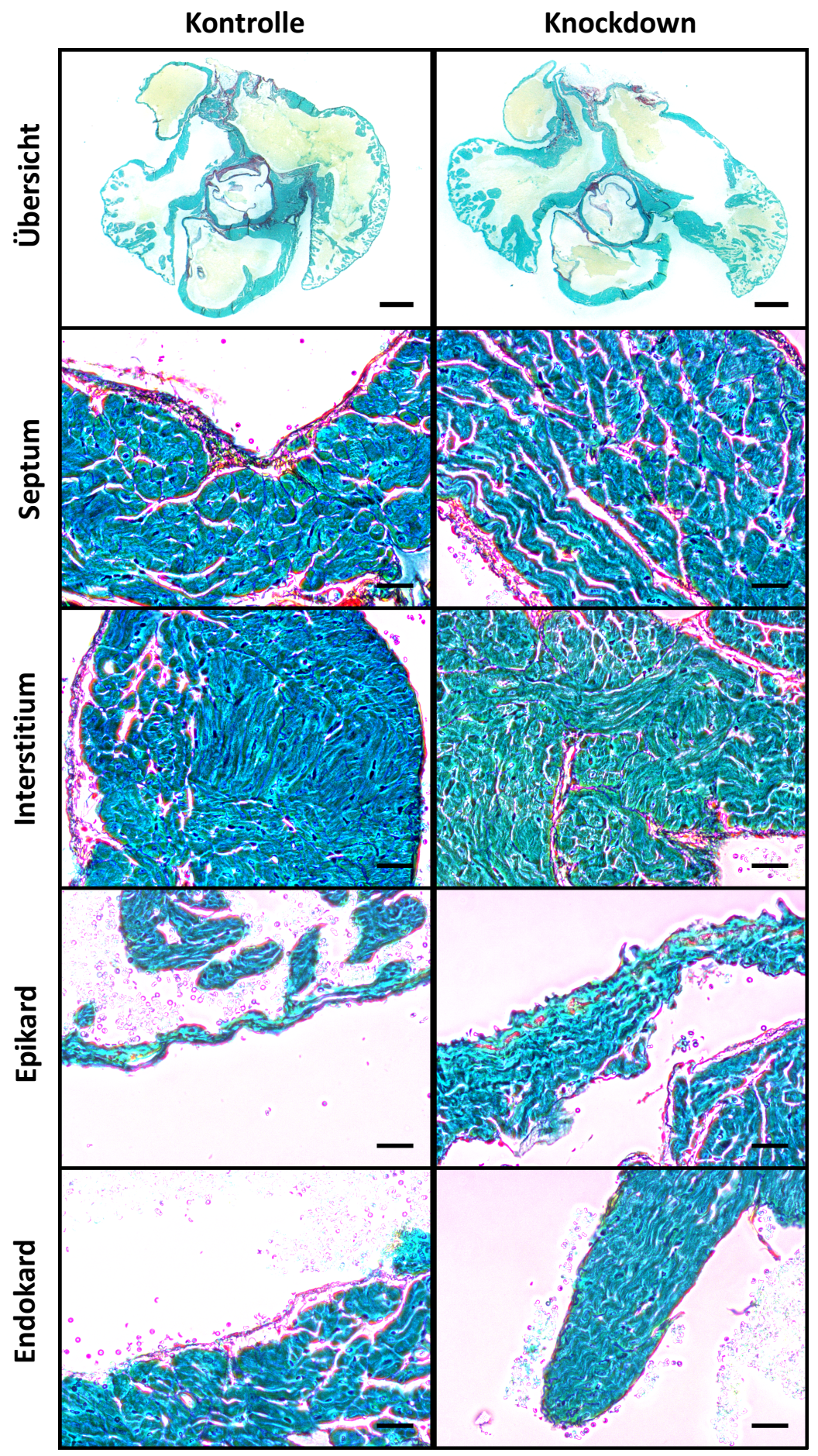

Abbildung 11: Sirius-Red/Fast-Green-Färbung zeigt keine atriale Fibrose nach Junctophilin-

\section{2-Knockdown.}

Zu Abbildung 10 analoge Darstellung der Schnitte mit Sirius-Red/Fast-Green-Färbung. Rot angefärbte Kollagenfasern nahmen in der Knockdown-Gruppe nicht zu. Maßstäbe: Übersicht $500 \mu \mathrm{m}$, Rest $50 \mu \mathrm{m} ; \mathrm{n}=3 / 3$ Herzen. 
Bei Aktivierung lokaler Fibroblasten, zum Beispiel durch mechanischen Stress, können diese proliferieren und Kollageneinlagerungen im Gewebe bewirken, die die Durchblutung und elektromechanische Effizienz des Myokards kompromittieren. So entsteht bei Einlagerung ins Interstitium eine interstitielle Fibrose, bei Einlagerung in die Adventitia der Koronargefäße eine perivaskuläre Fibrose (Talman und Ruskoaho 2016; Weber et al. 2013).

Zur histochemischen Detektion gewebsständiger Kollagenfasern und der Abgrenzung zu umgebendem Gewebe wurden histologische Anschnitte wie in 3.5.1 beschrieben angefertigt und mit den Farbstoffen Sirius Red und Fast Green behandelt. Sirius Red bindet spezifisch die $[\mathrm{Gly}-\mathrm{X}-\mathrm{Y}]_{\mathrm{n}}-$ Helixstruktur fibrillärer Kollagene, unabhängig vom Kollagentyp oder der Spezies. Fast Green bindet nicht-kollagenöse Proteine (Segnani et al. 2015).

In der Übersichtsaufnahme gab es visuell keinen Unterschied zwischen Kontroll- und Knockdown-Gruppe in Bezug auf den Flächenanteil rot gefärbter Kollagenfasern (siehe Abbildung 11). Die vergrößerten Ausschnitte von Septum, Interstitium, Epikard und Endokard gaben ebenfalls keinen Anhalt für interstitielle oder perivaskuläre Fibrose. Auch perikapillär gab es keine zusätzliche Kollageneinlagerung. Hiermit konnte eine reaktive Fibrose als unspezifische Ursache der oben beschriebenen echokardiographisch festgestellten atrialen Dysfunktion (siehe 3.2) ausgeschlossen werden.

\subsection{Konfokalmikroskopische und STED-Aufnahmen fixierter Kardiomyozyten mittels Immunfluoreszenzfärbungen}

Alle Aufnahmen in diesem Kapitel wurden an einem STED-Mikroskop (TCS SP8 STED, HC PL APO CS2 100x/1.4 Öl-Objektiv, Leica Microsystems) erstellt. Hierfür wurden murine VM und AM isoliert (siehe 2.5), fixiert und verschiedenen Protokollen der Immunfärbung unterzogen (siehe 2.9.1). Humane Proben stammten von Patienten mit Sinusrhythmus, denen während der Bypass-Operation atriales Gewebe entnommen wurde, nachdem sie infolge adäquater Aufklärung dieser Prozedur schriftlich zugestimmt hatten (siehe 2.9.2).

\subsubsection{Charakterisierung atrialer Kardiomyozyten der Maus und des Menschen}

Zunächst wurde analog zu den proteinbiochemischen Experimenten untersucht, wie sich die JPH2-Proteinexpression im murinen Wildtyp zwischen VM und AM unterscheidet. Abbildung 12 zeigt entsprechende STED-Aufnahmen.

Wie auch im Western-Blot (siehe 3.1) wurde eine in AM verminderte JPH2Proteinexpression festgestellt. Zudem waren die JPH2-Cluster in VM durchweg groß und regulär entlang der Z-Linien ausgerichtet, während sich in AM große Cluster eher perlschnurartig axial anordneten, in etwa parallel zur longitudinalen Zellachse. Nur vereinzelte kleine JPH2-Cluster befanden sich der Querstreifung entsprechend entlang der Z-Linien. 


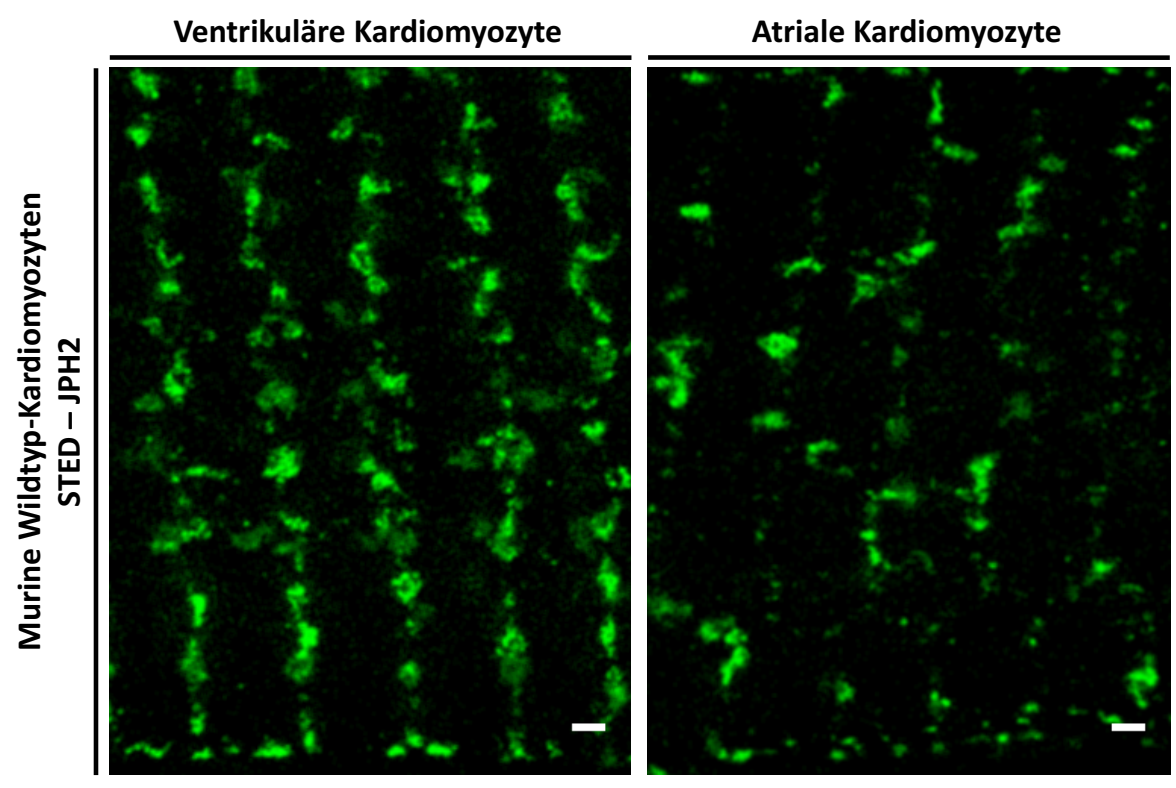

Abbildung 12: Vergleich der Größe, Abundanz und Verteilung ventrikulärer und atrialer Junctophilin-2-Cluster anhand von STED-Aufnahmen (muriner Wildtyp).

Ventrikuläre Myozyten bilden hauptsächlich große Junctophilin-2(JPH2)-Cluster entlang der Querstreifung aus. Atriale Myozyten zeigen vor allem große axial angeordnete und daneben kleine transversal angeordnete Cluster-Populationen. Generell geringere JPH2-Proteinexpression im Atrium im Vergleich zum Ventrikel. Maßstab: $1 \mu \mathrm{m}$.

Die zur weiteren Charakterisierung von Wildtyp-AM angefertigten Aufnahmen der Tripelfärbung gegen RyR2-pS2808/RyR2/JPH2 (siehe Abbildung 13A) belegen, dass diese groBen, axial ausgerichteten JPH2-Cluster mit hochphosphorylierten RyR2-pS2808-Clustern im Zentrum der Zelle kolokalisieren (violette Punkte), während deutlich kleinere JPH2-Cluster mit entlang der Z-Linien ausgerichteten Cluster-Ketten von niedrigphosphoryliertem RyR2 überlappen (türkise Punkte). Der Großteil dieser nicht-junktionalen niedrigphosphorylierten RyR2-Cluster wird von deutlich geringerem JPH2-Signal überlagert und erscheint daher grün, kreuzt aber regelmäßig axiale Perlschnurketten aus JPH2 und RyR-pS2808. Aus ZStacks angefertigte 3D-Modelle von Wildtyp-AM, jeweils gefärbt gegen RyR2/JPH2 bzw. RyR2/RyR2-pS2808 (siehe Abbildung 13B) veranschaulichen - durch die ganze Zelle betrachtet - ein vergleichbares Verteilungsmuster von JPH2 und RyR2-pS2808 in Relation zu RyR2.

Auch humane AM bilden axial ausgerichtete Ketten großer JPH2-Cluster aus: Abbildung 14 zeigt den histologischen Schnitt eines humanen Atriums, der mit der Ko-Färbung gegen JPH2/Cav3 behandelt wurde. Caveolin-3 diente hier als Marker für Tubulusmembranstrukturen innerhalb des TAT-Netzwerks. Deutlich ist eine alternierende axiale Anordnung grüner Cav3-Cluster und roter JPH2-Cluster zu erkennen, die sich in der Überlagerung gelb darstellen. Große JPH2-Cluster, die mit RyR2-pS2808 kolokalisieren, stehen demnach in direktem Kontakt zu AT. 
A
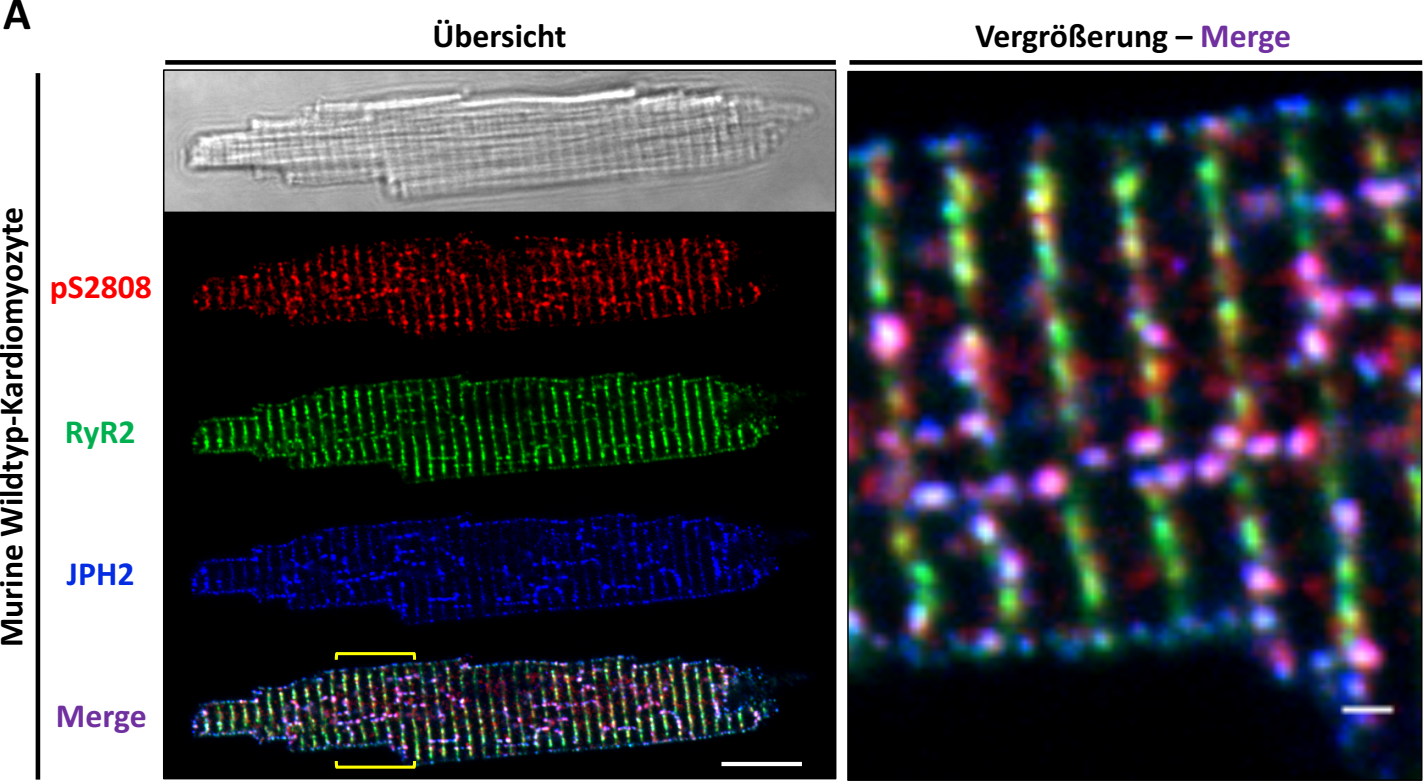

B

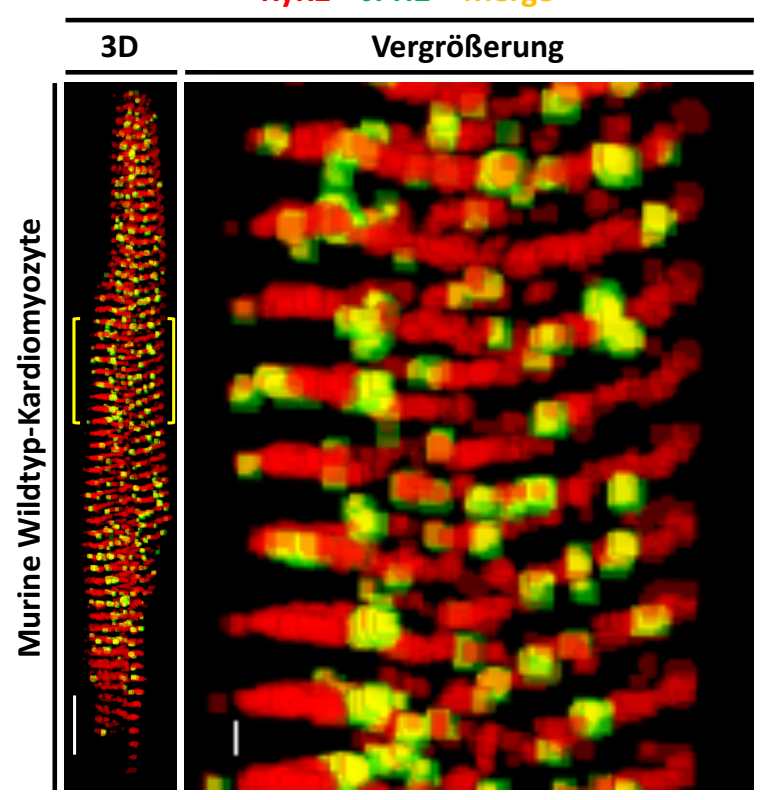

RyR2 - JPH2 - Merge

RyR2 - pS2808 - Merge - (Nukleus)

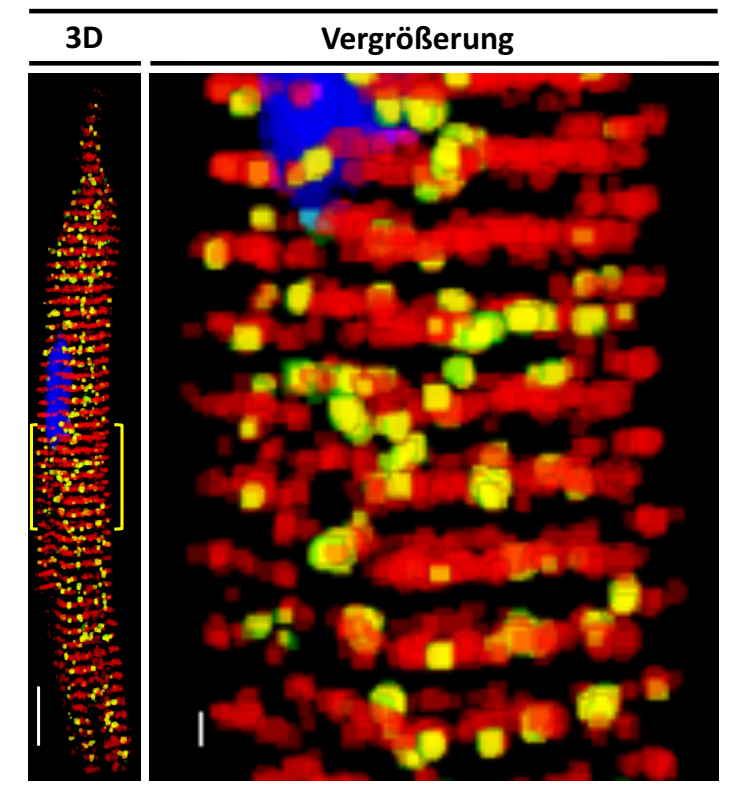

Abbildung 13: Konfokale Aufnahmen der RyR2-pS2808/RyR2/JPH2-Färbung illustrieren sowohl die Kolokalisation großer Junctophilin-2-Cluster mit Clustern des hochphosphorylierten Ryanodin-Rezeptors Typ 2 als auch deren ähnliches Verteilungsmuster innerhalb der Zelle.

(A) Links Darstellung konfokaler Übersichtsaufnahmen der atrialen Myozyte einer Wildtyp-Maus, getrennt nach den Kanälen Durchlicht, PKA-abhängig Serin-2808-phosphorylierter RyanodinRezeptor Typ 2 (pS2808) in rot, Ryanodin-Rezeptor Typ 2 (RyR2) in grün, Junctophilin-2 (JPH2) in blau und Überlagerung (Merge). Rechts Vergrößerung des gelb eingeklammerten Bereiches. Maßstäbe: Übersicht $10 \mu \mathrm{m}$, Vergrößerung $1 \mu \mathrm{m}$. (B) 3D-Rekonstruktionen konfokaler Z-Stacks. Links Darstellung der konfokalen Übersichtsaufnahme inklusive Vergrößerung (Bereich der gelben Klammer) der atrialen Myozyte einer Wildtyp-Maus, wobei die Kanäle RyR2 (rot) und JPH2 (grün) überlagert wurden (Merge). Rechts analoge Darstellung der subzellulären Strukturen in einer anderen Zelle, die im grünen Kanal den PKA-abhängig an Serin-2808 phosphorylierten Ryanodin-Rezeptor 
Typ 2 (pS2808) zeigt, zusätzlich den Nukleus in blau. Maßstäbe: 3D $10 \mu \mathrm{m}$, Vergrößerung $1 \mu \mathrm{m}$. Abstand der Schnitte in der Z-Achse: 0,44 $\mu \mathrm{m}$.

Humanes atriales Gewebe

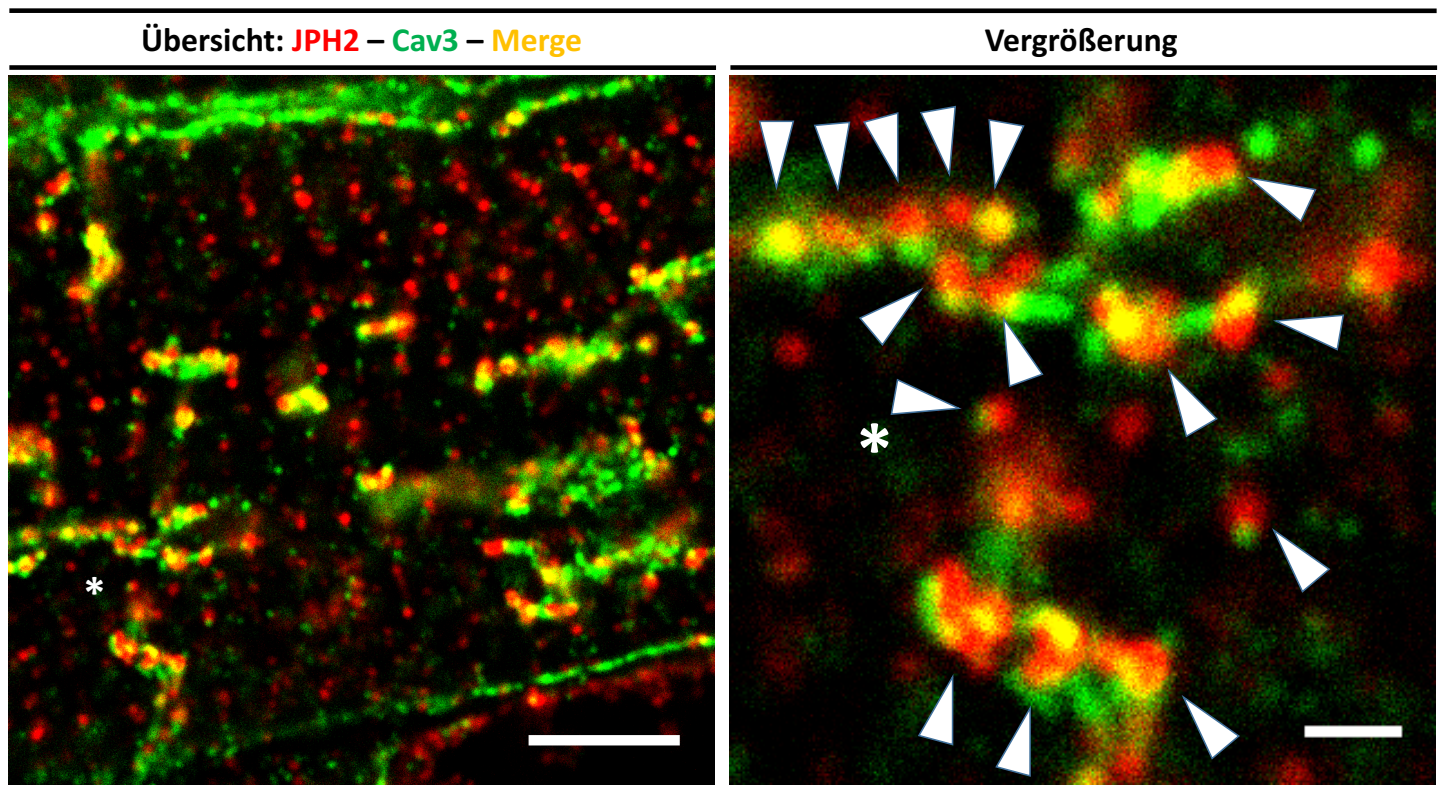

Abbildung 14: Konfokalmikroskopisch nachweisliche Kopplung großer Junctophilin-2Cluster an axiale Tubuli in humanen atrialen Kardiomyozyten.

Links Übersichtsbild einer humanen atrialen Myozyte mit den angefärbten Proteinen Junctophilin-2 (JPH2, rot) und Caveolin-3 (Cav3, grün), Überlagerungen (Merge) in gelb. Rechts eine Vergrößerung des markierten Bereichs (Asterisk). Die Pfeilspitzen zeigen exemplarisch auf große JPH2-Cluster, die sich in großer Nähe von Tubulusmembranstrukturen (Cav3-Signale) befinden. Maßstäbe: Übersicht $5 \mu \mathrm{m}$, Vergrößerung $1 \mu \mathrm{m}$.

Diese Daten belegen spezifische Membrandomänen von JPH2 in isolierten AM und legen eine differenzielle Verteilung von JPH2-Clustern innerhalb der Zelle nahe: Die Kolokalisation großer junktionaler JPH2-Cluster mit PKA-phosphoryliertem RyR2 entlang AT tief innerhalb AM könnte eine Schlüsselrolle für JPH2 in den erst kürzlich entdeckten atrialen Super-hubs bedeuten (Brandenburg et al. 2016a), die im Folgenden durch Untersuchung des JPH2-Knockdowns in isolierten AM beleuchtet werden soll.

Die folgenden Ergebnisse beziehen sich stets auf den Vergleich zwischen AM der Kontroll- und der Knockdown-Gruppe. Von jeweils drei Mäusen beider Gruppen wurden 2p40mg AM isoliert, fixiert und fünf verschiedenen Protokollen entsprechend gefärbt: KoFärbungen gegen 1) JPH2/RyR2, 2) JPH2/Cav3, 3) RyR2-pS2808/RyR2, 4) RyR2pS2808/Cav3 und 5) Cav1.2/Cav3. Für die Protokolle 1), 2) und 5) wurden konfokale und hochauflösende STED-Aufnahmen angefertigt, um Analysen der Cluster-Populationen durchführen zu können, für 3) und 4) konfokale Aufnahmen zum Vergleich der Signalintensität. 


\subsubsection{Gestörtes Cluster-Verhalten junktionaler Typ-2-Ryanodin-Rezeptoren nach Junctophilin-2-Knockdown}

Zur grundlegenden Charakterisierung des JPH2-Knockdowns wurden gegen JPH2 und RyR2 gefärbte AM in der Übersicht konfokal und in der Vergrößerung via STED dargestellt (siehe Abbildung 15). Im Übersichtsbild der Knockdown-Gruppe ist die globale Signalminderung von JPH2 zu erkennen. Hochauflösende STED-Aufnahmen zeigen in der Vergrößerung, dass vor allem axial ausgerichtete JPH2-Cluster-Ketten an Größe verloren. Auch RyR2-Cluster waren in der Knockdown-Gruppe kleiner. Entsprechend nahm der prozentuale Flächenanteil aller RyR2-Cluster bezogen auf die Zellfläche ab (siehe Tabelle 11). Der Prozentsatz der mit JPH2 überlappenden RyR2-Cluster nahm im Mittel um 7,4 \% ab, dadurch ergab sich eine um $0,027 \mu \mathrm{m}^{2}$ kleinere Überlappungsfläche von RyR2- mit JPH2Signal. Erstaunlicherweise blieb der Flächenanteil von JPH2 trotz geringerer Proteinexpression und sichtbarer konfokaler Signalminderung gleich, möglicherweise durch räumliche Dissoziation großer JPH2-Cluster in zahlreiche kleinere Exemplare abseits von AT, oder durch gleichbleibende Cluster-Größe bei abnehmender Cluster-Dichte.

Tabelle 11: Ergebnisse der Cluster-Analyse.

\begin{tabular}{|c|c|c|c|c|}
\hline \multicolumn{5}{|c|}{$2 \mathrm{p} 40 \mathrm{mg}$} \\
\hline \multicolumn{2}{|l|}{ Cluster-Parameter } & Kontrolle & Knockdown & $p$ \\
\hline \multirow{2}{*}{ Flächenanteil (\%) } & JPH2 & $4,11 \pm 0,34$ & $4,23 \pm 0,43$ & 0,83 \\
\hline & RyR2 & $8,29 \pm 0,26$ & $6,65 \pm 0,27$ & 0,00007 \\
\hline \multirow{2}{*}{ Überlappung (\%) } & $\mathrm{JPH} 2$ & $51,23 \pm 1,63$ & $52,97 \pm 2,91$ & 0,61 \\
\hline & RyR2 & $48,81 \pm 2,49$ & $41,41 \pm 2,34$ & 0,039 \\
\hline \multirow{2}{*}{ Überlappung $\left(\mu \mathrm{m}^{2}\right)$} & JPH2 & $0,056 \pm 0,005$ & $0,065 \pm 0,003$ & 0,13 \\
\hline & RyR2 & $0,060 \pm 0,004$ & $0,033 \pm 0,003$ & 0,000007 \\
\hline
\end{tabular}

Mittelwert \pm SEM. Siehe Legende der Abbildung 15 für Abkürzungen und Erläuterungen.

Zusammengefasst bewirkte der JPH2-Knockdown eine Störung der Kolokalisation von junktionalem RyR2 mit JPH2 entlang axialer Cluster-Ketten im Zentrum der Zelle, die im Zusammenhang mit AT stehen. Zwar nahmen AT in der Knockdown-Gruppe zu, allerdings nahm proteinbiochemisch der Anteil des PKA-phosphorylierten RyR2-pS2808 ab, das normalerweise in Clustern entlang AT nachzuweisen ist (siehe auch 3.6.4 und 3.6.5). 
A

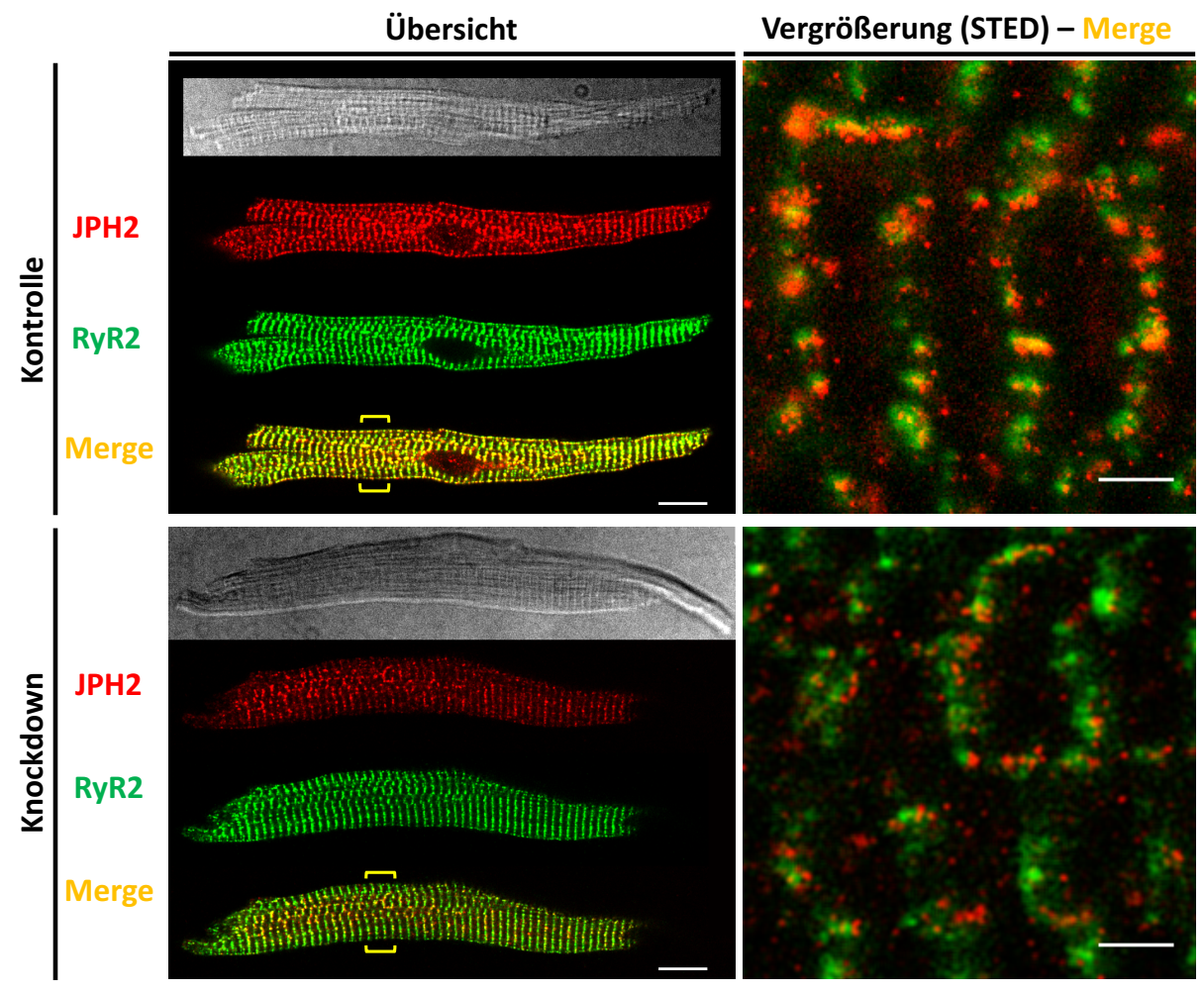

B

Vergrößerung (STED) - JPH2

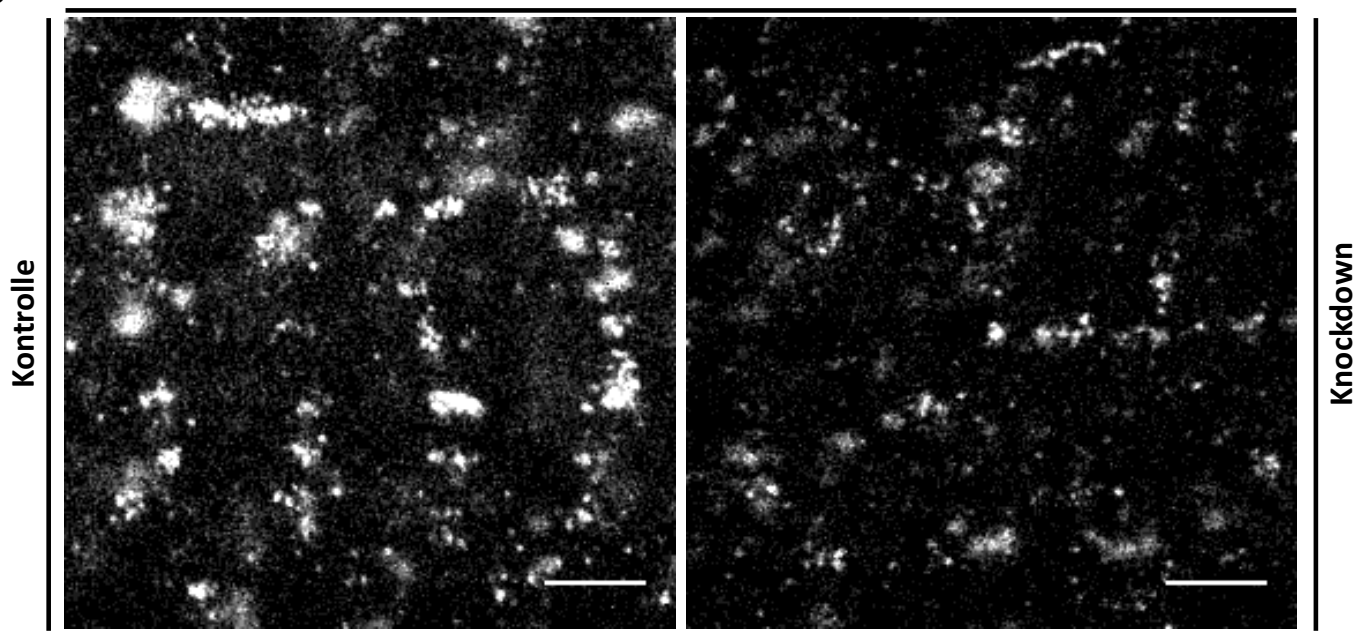

C Flächenanteil der Cluster

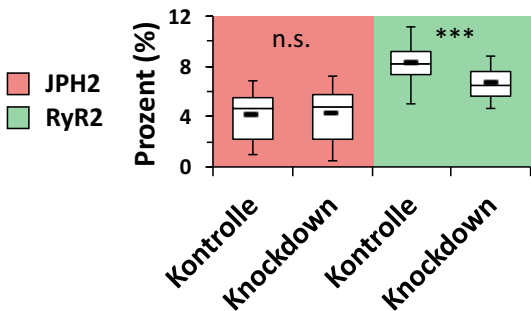

Überlappende Cluster

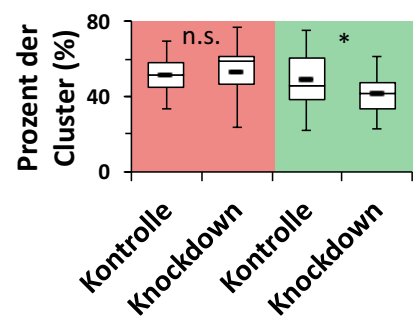

Überlappende Cluster
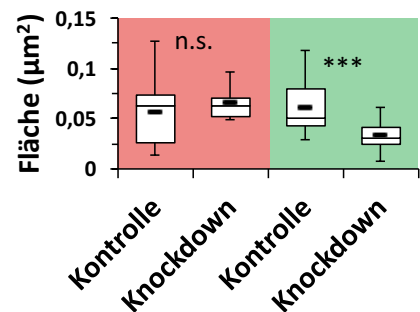

Abbildung 15: Konfokale und STED-Aufnahmen der JPH2/RyR2-Färbung illustrieren den Knockdown von Junctophilin-2 neben einem gestörten Cluster-Verhalten der Typ-2Ryanodin-Rezeptoren.

(A) Jeweils links Darstellung konfokaler Aufnahmen, getrennt nach den Kanälen Durchlicht, Junctophilin-2 (JPH2) in rot, Ryanodin-Rezeptor Typ 2 (RyR2) in grün und Überlagerung (Merge) in 
gelb. Rechts Vergrößerung aus dem gelb eingeklammerten Bereich, STED-Aufnahme. Maßstäbe: Übersicht $10 \mu \mathrm{m}$, Vergrößerung $1 \mu \mathrm{m}$. (B) Graustufen-Darstellung der STED-Aufnahmen des JPH2-Kanals aus (A) zur besseren Vergleichbarkeit. Die Größe der JPH2-Cluster (hauptsächlich axiale Cluster-Ketten) war in der Knockdown-Gruppe deutlich vermindert. Maßstab $1 \mu \mathrm{m}$. (C) Analyse der Cluster (JPH2 in rot, RyR2 in grün) in Bezug auf ihren Flächenanteil pro Zelle und Parameter der gegenseitigen Überlappung. RyR2-Cluster waren in der Knockdown-Gruppe flächenmäßig prozentual weniger vertreten. Ein geringerer Prozentsatz aller RyR2-Cluster überlappte mit JPH2-Clustern, und von RyR2 eingenommene Fläche in Überlagerung mit JPH2-Signal war signifikant vermindert. Interessanterweise war der prozentuale Flächenanteil von JPH2 in der Knockdown-Gruppe unverändert. Die Box-Plots repräsentieren den Median mit unterem und oberem Quartil, die Whisker das Minimum und Maximum, der kurze horizontale Strich das arithmetische Mittel; $n=30 / 22$ Zellen aus 3/3 Herzen; *: $p<0,05 ; * * *: p<0,001$; n.s.: nicht signifikant.

\subsubsection{Effekt des Junctophilin-2-Knockdowns auf junktionale Junctophilin-2-Cluster an axialen Tubuli}

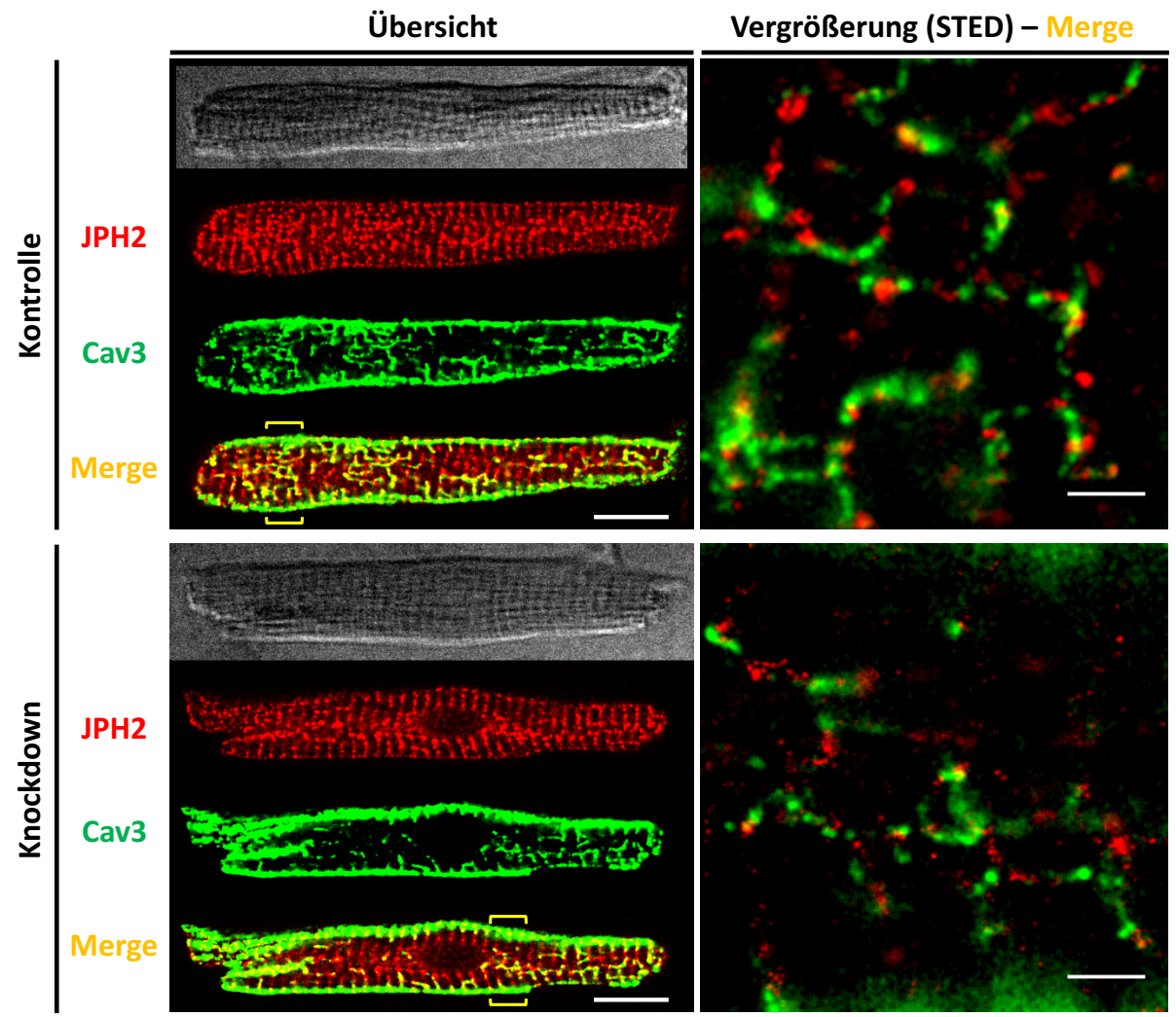

Abbildung 16: Konfokale und STED-Aufnahmen der JPH2/Cav3-Färbung zeigen eine verminderte Größe junktionaler Junctophilin-2-Cluster entlang axialer Tubuli.

Jeweils links Darstellung konfokaler Aufnahmen, getrennt nach den Kanälen Durchlicht, Junctophilin-2 (JPH2) in rot, Caveolin-3 (Cav3) in grün und Überlagerung (Merge) in gelb. Rechts VergröBerung aus dem gelb eingeklammerten Bereich, STED-Aufnahme. In der Vergrößerung der Knockdown-Aufnahmen sind im Vergleich zur Kontroll-Gruppe deutlich kleinere JPH2-Cluster zwischen den regelmäßig auftretenden Cav3-Clustern zu erkennen. Maßstäbe: Übersicht $10 \mu \mathrm{m}$, Vergrößerung $1 \mu \mathrm{m} ; \mathrm{n}=3 / 3$ Herzen. 
Die Ko-Färbung von AM gegen die Proteine JPH2 und Cav3 (siehe Abbildung 16) diente in diesem Zusammenhang der Bestätigung, dass sich große JPH2-Cluster entlang axialer (und vereinzelter transversaler) Tubulusmembranstrukturen anordnen und demnach junktional sind. Besonders in der hochauflösenden STED-Aufnahme ist zu erkennen, dass jeweils grüne (Cav3) und rote Cluster (JPH2) alternieren und sich seltener gelb dargestellt überlagern, als von der konfokalen Aufnahme her zu vermuten wäre. Kleinere nichtjunktionale JPH2-Cluster waren transversal entlang der Z-Linien ausgerichtet, ohne Kontakt zum TAT-Netzwerk. In der Knockdown-Gruppe waren besonders junktionale AT-assoziierte JPH2-Cluster verkleinert.

\subsubsection{Anordnung hochphosphorylierter Typ-2-Ryanodin-Rezeptoren in axialen} Cluster-Ketten
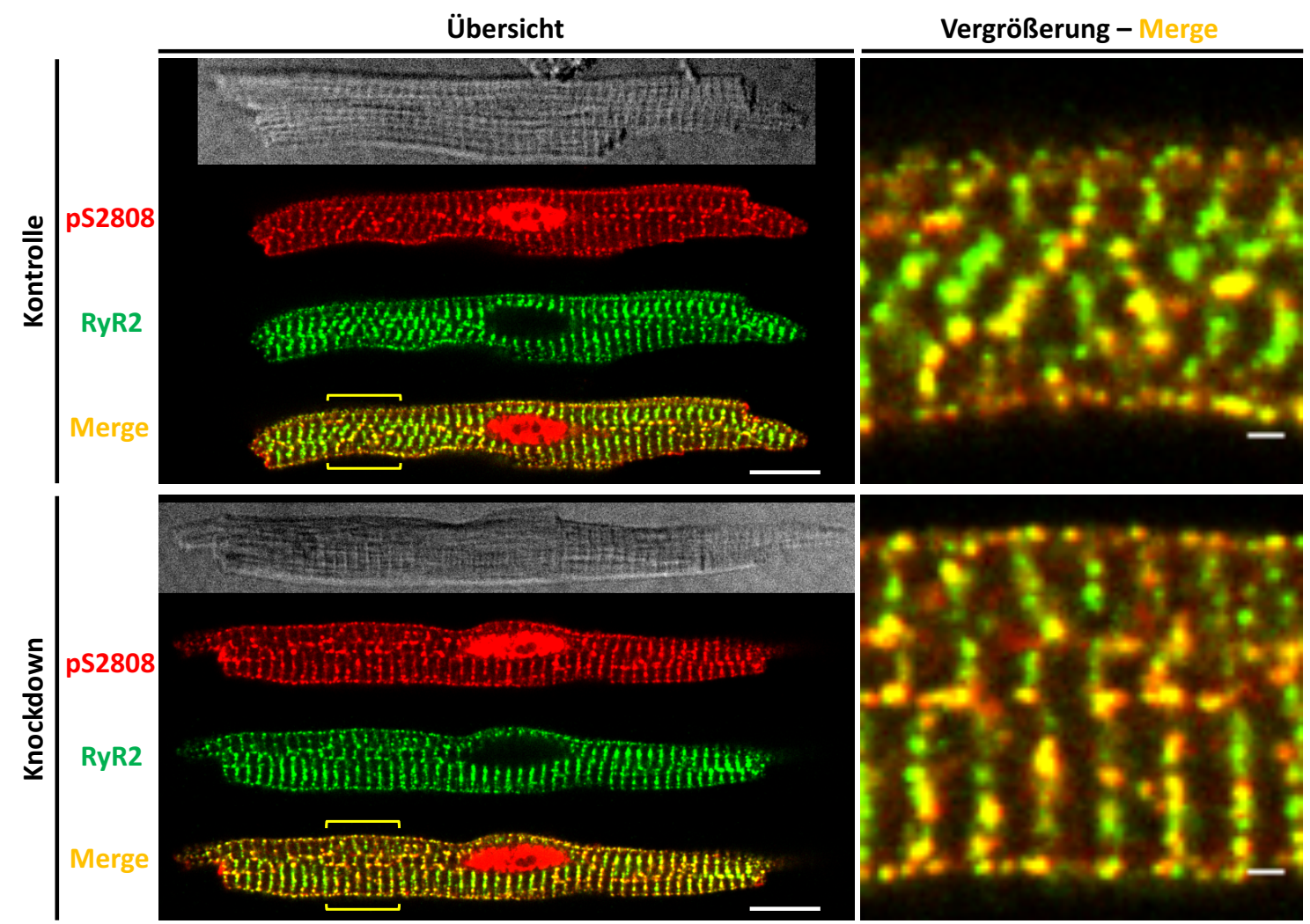

Abbildung 17: Konfokale Aufnahmen der RyR2-pS2808/RyR2-Färbung zeigen die differenzielle Verteilung niedrigphosphorylierter und hochphosphorylierter Typ-2Ryanodinrezeptoren in atrialen Myozyten.

Jeweils links Darstellung konfokaler Aufnahmen, getrennt nach den Kanälen Durchlicht, PKAabhängig Ser2808-phosphorylierter Ryanodin-Rezeptor Typ 2 (pS2808) in rot (Rotfärbung des Zellkerns als unspezifische Kreuzreaktion), Ryanodin-Rezeptor Typ 2 (RyR2) in grün und Überlagerung (Merge) in gelb. Rechts Vergrößerung des gelb eingeklammerten Bereiches. Maßstäbe: Übersicht $10 \mu \mathrm{m}$, Vergrößerung $1 \mu \mathrm{m} ; \mathrm{n}=3 / 3$ Herzen.

Konfokalmikroskopische Aufnahmen der Ko-Färbung gegen RyR2-pS2808/RyR2 (siehe Abbildung 17) zeigten keine Unterschiede in der Signalintensität dieser Proteine im Ver- 
gleich zwischen Knockdown- und Kontroll-Gruppe, obwohl das Verhältnis von RyR2pS2808 zu RyR2 proteinbiochemisch vermindert war. Klar erkennbar ist dennoch, dass sich RyR2-pS2808-Cluster selten entlang der transversalen Z-Streifung befanden, sondern besonders häufig in axial ausgerichteten Cluster-Ketten tief im Zellinneren ausgerichtet waren, wie schon in 3.6.1 beschrieben.

\subsubsection{Lokalisation hochphosphorylierter Typ-2-Ryanodin-Rezeptoren an axialen Tubuli des TAT-Netzwerks}

Abbildung 18 illustriert anhand konfokaler Aufnahmen exemplarisch die Ergebnisse der Ko-Färbung von AM gegen RyR2-pS2808 und Cav3. Als Ergänzung zur RyR2pS2808/RyR2-Färbung wurde hier bestätigt, dass sich die großen axial ausgerichteten Ketten von RyR2-pS2808-Clustern hauptsächlich in unmittelbarer Nähe von AT befanden, die mittels Cav3 markiert waren. Eine zusätzliche räumliche Assoziation von JPH2 mit RyR2pS2808, wie sie auch in Abbildung 13 (siehe 3.6.1) dargestellt ist, war demnach auch für AM der Kontroll- und Knockdown-Gruppe anzunehmen.

Zusätzlich ist am Cav3-Signal in Aufnahmen der Knockdown-Gruppe die in 3.4.2 beschriebene Zunahme von AT und der Dichte des TAT-Netzwerks ersichtlich.

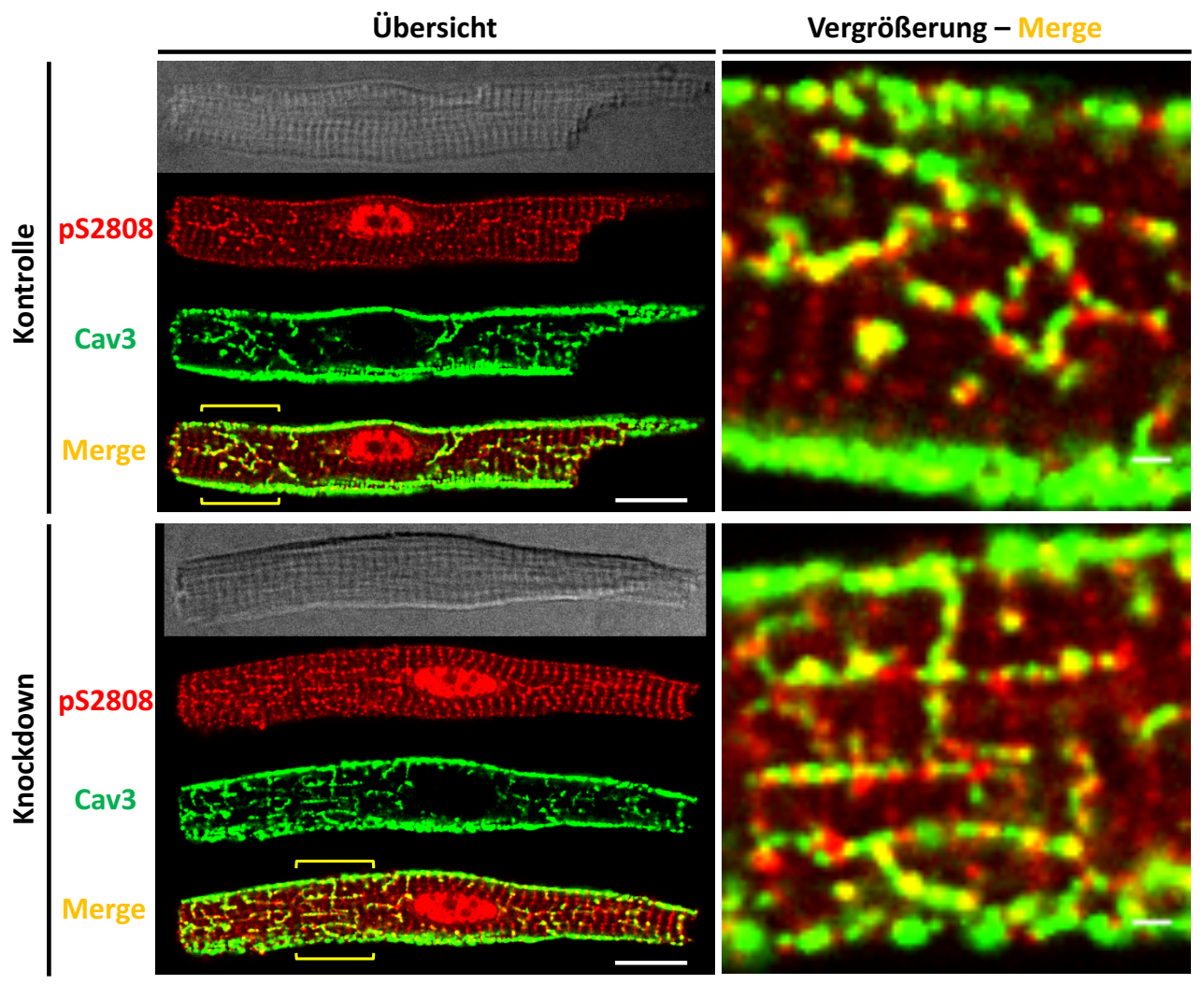

Abbildung 18: Konfokale Aufnahmen der RyR2-pS2808/Cav3-Färbung belegen die junktionale Eigenschaft hochphosphorylierter Typ-2-Ryanodin-Rezeptoren an axialen Tubuli.

Jeweils links Darstellung konfokaler Aufnahmen, getrennt nach den Kanälen Durchlicht, Serin2808-phosphorylierter Ryanodin-Rezeptor Typ 2 (pS2808) in rot (Rotfärbung des Zellkerns als unspezifische Kreuzreaktion), Caveolin-3 (Cav3) in grün und Überlagerung (Merge) in gelb. Rechts 
Vergrößerung des gelb eingeklammerten Bereiches. Maßstäbe: Übersicht $10 \mu \mathrm{m}$, Vergrößerung $1 \mu \mathrm{m} ; \mathrm{n}=3 / 3$ Herzen.

\subsubsection{Größenabnahme TAT-assoziierter Cav1.2-Cluster und zellweite Dispersion intrazellulärer Cav1.2-Cluster nach Junctophilin-2-Knockdown}

Räumlich unmittelbar gegenüber junktionalen SR-ständigen RyR2-Clustern befinden sich in der Tubulusmembran spannungsabhängige Cav1.2-Cluster (Wehrens et al. 2005), die für den Prozess der CICR essenziell sind (Fabiato 1983).

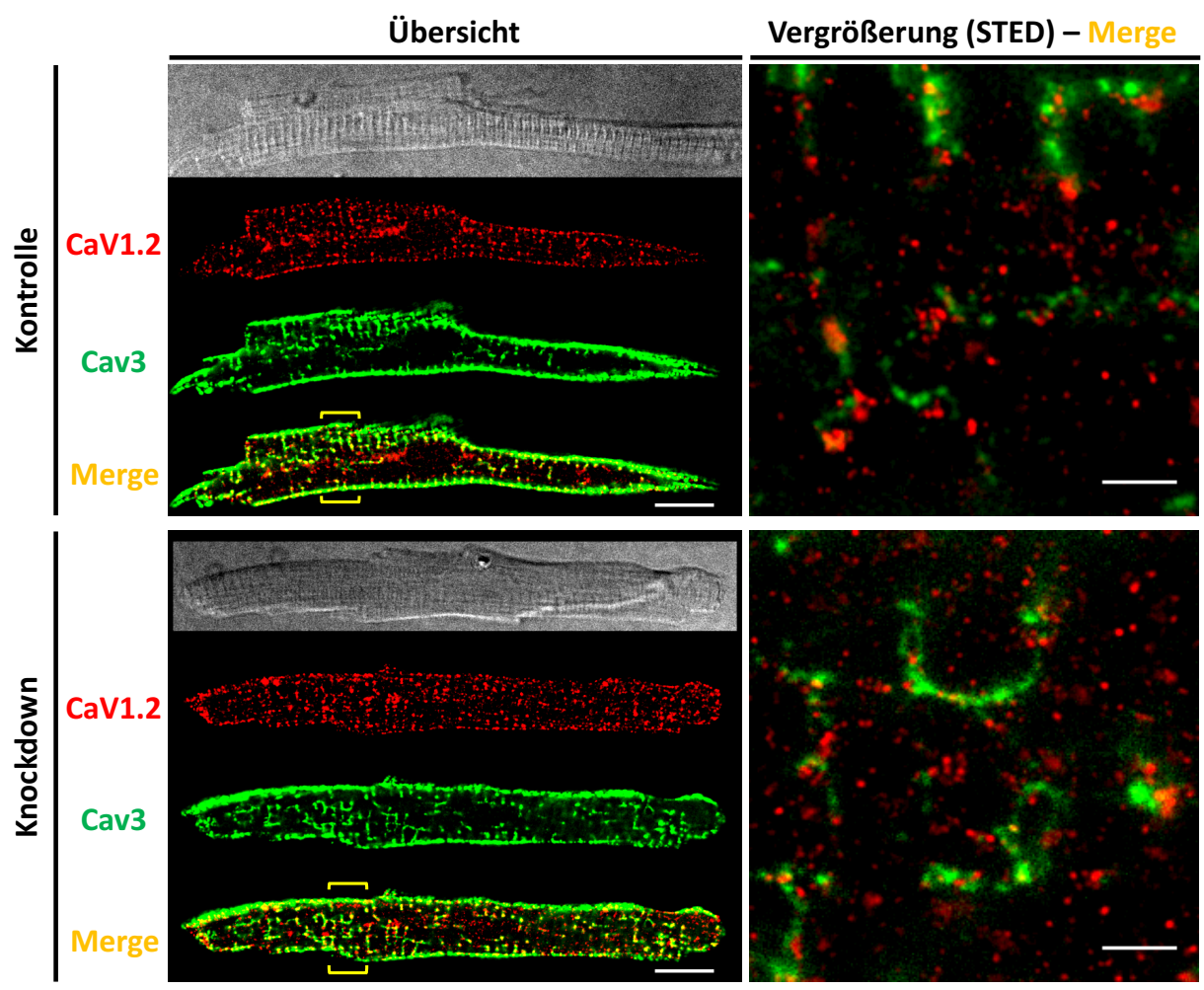

Abbildung 19: Konfokale und STED-Aufnahmen der Cav1.2/Cav3-Färbung zeigen die Lokalisation spannungsabhängiger L-Typ-Kalziumkanäle an Tubulusmembranstrukturen und Auswirkungen des Junctophilin-2-Knockdowns.

Jeweils links Darstellung konfokaler Aufnahmen, getrennt nach den Kanälen Durchlicht, spannungsabhängiger L-Typ-Kalziumkanal 1.2 (Cav1.2) in rot, Caveolin-3 (Cav3) in grün und Überlagerung (Merge) in gelb. Rechts Vergrößerung aus dem gelb eingeklammerten Bereich, STEDAufnahme. Große Cav1.2-Cluster waren mit Cav3-markierten Membranstrukturen assoziiert. Es ist davon auszugehen, dass es sich hierbei um funktionell auf Strukturen des TAT-Netzwerks exprimierte $\mathrm{Ca}_{\mathrm{v}} 1.2-$ Cluster handelt. Nach JPH2-Knockdown waren diese TAT-assoziierten Cluster verkleinert. Kleinere intrazellulär akkumulierte Cav1.2-Cluster waren im gesamten Zellbereich (Zellkern ausgenommen) vorhanden und ungeordnet verteilt. Maßstäbe: Übersicht $10 \mu \mathrm{m}$, Vergrößerung $1 \mu \mathrm{m} ; \mathrm{n}=3 / 3$ Herzen.

Abbildung 19 zeigt die Darstellung von Cav1.2 und Cav3 in der Kontroll- und KnockdownGruppe. In den hochauflösenden STED-Aufnahmen ist zu erkennen, dass sich ebenfalls im Falle von Cav1.2 große Cluster vor allem in direkter Nachbarschaft zu Cav3-markierten 
axialen Domänen befanden, ähnlich den Proteinen JPH2 und RyR2-pS2808. Es ist davon auszugehen, dass diese $\mathrm{Ca}_{\mathrm{v}} 1.2$-Cluster innerhalb des TAT-Netzwerks eine funktionelle Rolle haben. In Aufnahmen der Knockdown-Gruppe nahm die Größe dieser TAT-assoziierten Cav1.2-Cluster im Vergleich zur Kontroll-Gruppe ab, dagegen waren kleinere Cav1.2-Cluster abseits von Membranstrukturen in großer Abundanz in der Zelle verteilt.

\subsection{Erhöhte Herzfrequenz und verzögerte atrioventrikuläre Erregungsleitung nach Junctophilin-2-Knockdown}

Um funktionelle Veränderungen der Erregungsausbreitung bei atrialer Dysfunktion nach JPH2-Knockdown zu untersuchen, wurde bei einer Gruppe von Mäusen unter Isoflurannarkose (1,5 Vol.-\%) einen Tag vor der Herzentnahme ein 6-Kanal-EKG geschrieben (siehe 2.11). Die Messung fand zunächst zehn Minuten lang in Ruhe statt (BASAL). Zur Untersuchung der atrialen Stressreaktion wurde jedem Tier i.p. Isoproterenol injiziert, gefolgt von weiteren zehn Minuten Messung (ISO). Es wurden die Parameter Herzfrequenz, P-Welle, PQ-Intervall und PR-Intervall manuell und automatisiert ausgewertet. Wegen großer Unterschiede der Herzfrequenz wurden die Datensätze je für BASAL und ISO auf den Mittelwert der Herzfrequenz der Kontroll-Gruppe normalisiert.

Aus Abbildung 20 wird die Verzögerung der atrialen Erregungsleitung in der KnockdownGruppe ersichtlich. Zusätzlich zeigten Knockdown-Mäuse atrioventrikuläre Erregungsausbreitungsstörungen wie z. B. intermittierend einen junktionalen Ersatzrhythmus mit Aussetzen der P-Welle und schwankende PQ-Intervalle. Die Werte für Herzfrequenz, P-Welle, PQ-Intervall und PR-Intervall waren in der Knockdown-Gruppe BASAL signifikant erhöht. Nach $\beta$-adrenerger Stimulation durch ISO-Injektion verblieben signifikant höhere Werte nur im PQ- und PR-Intervall, während sich die Werte für Herzfrequenz und P-Welle anglichen.

Die softwareseitig automatisierte Analyse wich vor allem bei den Leitungszeiten der PWelle signifikant von der manuellen Analyse ab. Im Mittel erkannte sie deren Länge als um 4,4 ms (BASAL, $\mathrm{p}=$ 0,001) bzw. um 2,7 ms (ISO, $\mathrm{p}=0$,003) kürzer. Die Auswertung des PQ-Intervalls unterschied sich nur in der Kontroll-Gruppe BASAL um 4,9 ms ( $p=0,007)$. Das PR-Intervall nach ISO wurde automatisiert im Schnitt 3,3 ms länger $(p=0,004)$ als manuell gemessen. Die Unterschiede zwischen Kontroll- und Knockdown-Gruppe waren jedoch innerhalb der jeweiligen Art der Analyse konsistent.

Zusammengefasst führte der JPH2-Knockdown zu signifikanter Erhöhung der basalen Herzfrequenz mit Verzögerung der atrialen Erregung, die auch durch positiv dromotrope $\beta$ adrenerge Stimulation nicht vollständig kompensiert werden konnte. Vereinzelt kam es sogar zum Aussetzen der P-Welle mit junktionalem Ersatzrhythmus als Hinweis für eine stark verzögerte Impulsausbreitung vom Vorhof zum Ventrikel. 

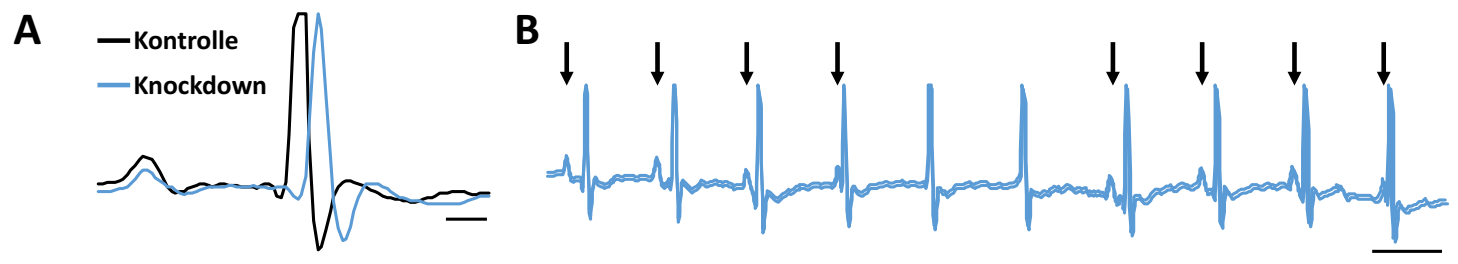

\section{Herzfrequenz}
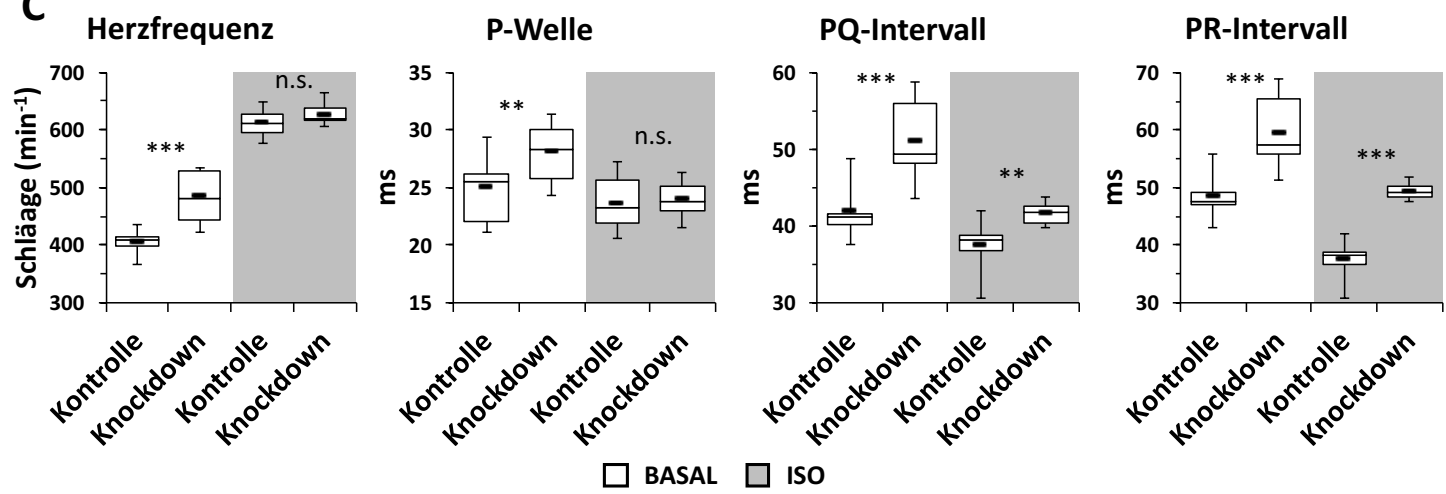

D Herzfrequenz
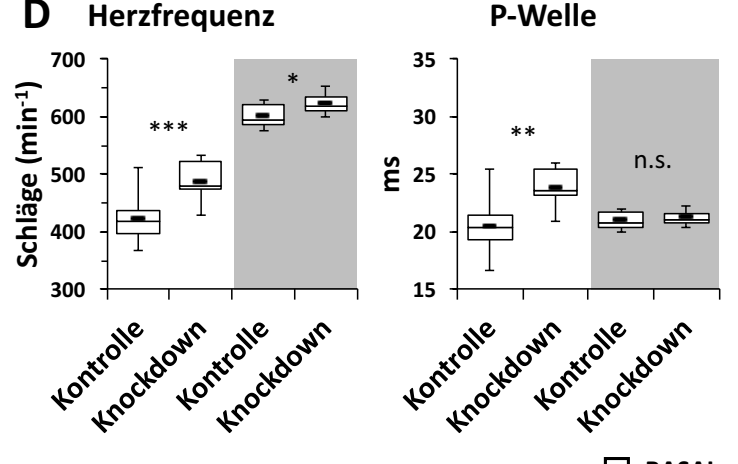

PQ-Intervall

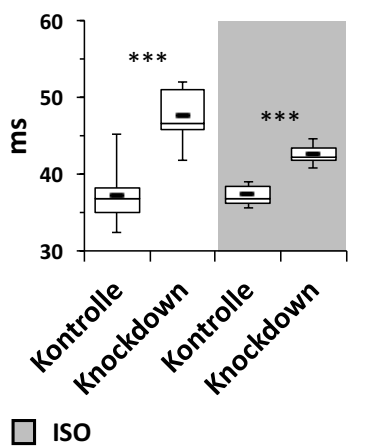

PR-Intervall

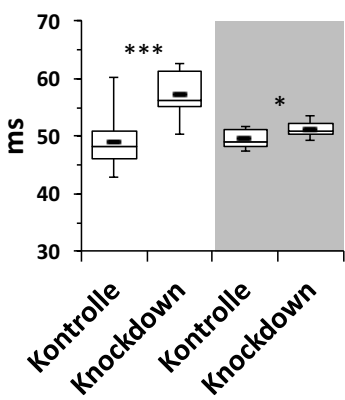

Abbildung 20: Verzögerte atrioventrikuläre Erregungsleitung bei erhöhter Herzfrequenz nach Junctophilin-2-Knockdown.

(A) Repräsentative Herzaktionen aus Ableitung I (BASAL) zeigen atriale Erregungsverzögerung in der Knockdown-Gruppe; Maßstab: 10 ms. (B) Ausschließlich bei Mäusen der Knockdown-Gruppe wurde BASAL intermittierend ein junktionaler Ersatzrhythmus beobachtet. Pfeile: P-Welle; Maßstab: 100 ms. (C) Manuelle Analysen zeigten in der Knockdown-Gruppe BASAL eine erhöhte Herzfrequenz sowie eine verlängerte P-Welle und PQ-Zeit. Nach Isoproterenol-Injektion (ISO) normalisierte sich die P-Welle. Die PQ-Zeit blieb im Vergleich zur Kontroll-Gruppe signifikant verlängert. (D) Automatisierte Analysen des Datensatzes. Im Vergleich zwischen Kontroll- und Knockdown-Gruppe gleiche Verhältnisse wie in (C). Die Daten wurden für BASAL und ISO je auf den Mittelwert der Herzfrequenz der Kontroll-Gruppe normalisiert. Die Box-Plots repräsentieren den Median mit unterem und oberem Quartil, die Whisker das Minimum und Maximum, der kurze horizontale Strich das arithmetische Mittel; $\mathrm{n}=10 / 13$; * $\mathrm{p}<0,05 ; *^{*}: \mathrm{p}<0,01 ; *^{* *}: \mathrm{p}<0,001$; n.s.: nicht signifikant. 
Tabelle 12: Messwerte der Elektrokardiographie.

\begin{tabular}{l|l|l|llll}
\hline \hline & \multicolumn{3}{|c|}{ BASAL } & \multicolumn{3}{c}{ ISO } \\
\hline Parameter & Kontrolle & Knockdown & $\mathrm{p}$ & Kontrolle & Knockdown & $\mathrm{p}$ \\
\hline
\end{tabular}

MA N UELLE A U S W E T U N G (Abbildung 20C)

\begin{tabular}{l|ll:l|ll:l}
\hline HF (min-1) & $405,91 \pm 7,60$ & $484,65 \pm 14,04$ & 0,0002 & $612,07 \pm 8,0$ & $626,63 \pm 5,79$ & 0,16 \\
P-Welle (ms) & $24,96 \pm 0,56$ & $28,09 \pm 0,83$ & 0,007 & $23,59 \pm 0,54$ & $23,94 \pm 0,51$ & 0,65 \\
PQ-Intervall (ms) & $41,96 \pm 0,97$ & $51,02 \pm 1,73$ & 0,0004 & $37,59 \pm 1,09$ & $41,61 \pm 0,45$ & 0,006 \\
PR-Intervall (ms) & $48,63 \pm 1,0$ & $59,50 \pm 1,99$ & 0,0002 & $44,63 \pm 0,94$ & $49,41 \pm 0,46$ & 0,0004 \\
\hline
\end{tabular}

A UTOMAT IS I ERTE A U SWERT N G (Abbildung 20D)

\begin{tabular}{l|ll:l|ll:l}
\hline HF (min-1) & $421,17 \pm 11,92$ & $487,59 \pm 8,97$ & 0,0005 & $601,32 \pm 6,21$ & $622,24 \pm 4,16$ & 0,02 \\
P-Welle (ms) & $20,46 \pm 0,71$ & $23,79 \pm 0,43$ & 0,002 & $20,95 \pm 0,22$ & $21,22 \pm 0,14$ & 0,34 \\
PQ-Intervall (ms) & $37,09 \pm 1,05$ & $47,51 \pm 0,89$ & $7 \mathrm{E}-7$ & $37,29 \pm 0,39$ & $42,55 \pm 0,28$ & $6 \mathrm{E}-9$ \\
PR-Intervall (ms) & $48,95 \pm 1,42$ & $57,20 \pm 1,06$ & 0,0003 & $49,45 \pm 0,51$ & $51,17 \pm 0,34$ & 0,02 \\
\hline \hline
\end{tabular}

Mittelwerte \pm SEM. HF: Herzfrequenz. Siehe Legende der Abbildung 20 für Erläuterungen. 


\section{Diskussion}

Diese Arbeit hat erstmals in AM untersucht, ob und wie das essenzielle SR-Protein JPH2 subzelluläre junktionale Membrankontakte mit TAT-Membranstrukturen formt und stabilisiert. Für diese Fragestellung wurde ein Knockdown-Protokoll gegen JPH2 in einem transgenen Mausmodell etabliert, das durch Tamoxifen-induzierte shRNA-Expression in zwei Wochen zu einer signifikant verminderten Proteinexpression von JPH2 im Atrium und Ventrikel führte. Die Arbeit baut auf einem neuen zellbiologischen Modell des atrialen TAT-Netzwerks auf, welches eine Super-bub-artige Auslösung der $\mathrm{Ca}^{2+}$-Freisetzung aus dem SR durch TAT-assoziierte RyR2-Cluster für fünf Spezies von der Maus bis zum Menschen vorhersagt (Brandenburg et al. 2016a; Brandenburg et al. 2018). Da JPH2 bei Herzerkrankungen proteolytisch bedingt abnimmt, eröffnet das Mausmodell des shRNA-vermittelten Knockdowns von JPH2 mechanistische Untersuchungen atrialer Dysfunktion sowohl in vitro als auch in vivo.

\subsection{Expression von Junctophilin-2 im Atrium}

Bislang war nicht bekannt, ob die Atrien JPH2 in ähnlichem Maße exprimieren wie die Ventrikel. Die proteinbiochemische Analyse muriner Wildtyp-Proben ergab eine etwa fünffach niedrigere atriale JPH2-Proteinexpression verglichen mit dem Ventrikel (siehe 3.1). Aufgrund der im Vergleich mit VM um etwa $24 \%$ geringeren atrialen TATNetzwerkdichte (Brandenburg et al. 2016a) war die Hypothese möglich, dass in Atrien stöchiometrisch weniger JPH2 für die Kopplung des SR an die Tubulusmembran benötigt werde. Allerdings ließ sich proteinbiochemisch in AM und VM eine vergleichbar hohe RyR2-Expression nachweisen, so dass im Atrium nur etwa jedes vierte RyR2-Molekül von einem JPH2-Molekül funktionell stabilisiert werden kann. Interessanterweise zeigte sich in Immunfluoreszenzaufnahmen eine differenzielle Verteilung von JPH2-Clustern in eine junktionale und eine nicht-junktionale Gruppe: Junktionales JPH2 war überwiegend mit PKAhochphosphoryliertem RyR2-pS2808 in großen Clustern entlang AT im Zentrum der Zelle verteilt, während nicht-junktionales JPH2 ohne Kontakt zum TAT-Netzwerk mit niedrigphosphorylierten kleineren RyR2-Clustern entlang der transversalen Z-Streifung assoziiert war (siehe 3.6.1 und 4.3). Wir stellten folglich die Hypothese auf, dass AM, die auf eine schnellere Kontraktionsaktivierung angewiesen sind, durch eine verminderte JPH2/RyR2Stöchiometrie nach JPH2-Knockdown anfällig für eine Störung der JMC sein könnten.

\subsubsection{Neuartiger kardialer Knockdown von Junctophilin-2}

Diese Arbeit erweitert ein Mausmodell von van Oort et al. (2011), in dem die JPH2Expression adulter Mäuse des C57BL/6J-Hintergrunds durch Tamoxifen-induzierte shRNA gegen JPH2 vermindert wurde. Im Gegensatz zum Knockdown führte der globale 
Knockout von JPH2 schon in der Embryonalentwicklung zum Tod (Takeshima et al. 2000). Der shRNA-vermittelte Knockdown von JPH2 war im Atrium bisher nicht untersucht worden. Wir konnten 2p40mg und 4p40mg proteinbiochemisch JPH2-Expressionslevel feststellen, die im Mittel zu über 50 \% im Vergleich zur Kontroll-Gruppe (wie im Methodenteil beschrieben, siehe 2.1) reduziert waren, jedoch ohne jegliche Mortalität (siehe 3.1.1). Durch das neue Tamoxifen-Induktionsprotokoll, das sich durch einmalige i.p.-Injektion und geringere Gesamtdosis (40 mg/kg Körpergewicht) auszeichnet, wurde eine wesentlich weniger belastende Knockdown-Untersuchung in der Maus ermöglicht. Aufgrund der atrial und ventrikulär unterschiedlichen Ausgangsmenge an JPH2-Protein ergab sich in der atrialen Knockdown-Gruppe 2p40mg eine ca. fünffach geringere JPH2-Proteinexpression verglichen mit gesunden VM. Im Gegensatz dazu führte das 2p100mg Protokoll zwar auch zu einer signifikanten JPH2-Proteinreduktion, allerdings lag die Mortalität der Knockdown-Gruppe vergleichbar zur ventrikulären Vorgängerstudie (van Oort et al. 2011) bei $40 \%$, was auf eine zu große, von uns nicht intendierte Krankheitsbelastung schließen ließ. Die finalen Behandlungsbedingungen für alle weitere Untersuchungen der Auswirkungen des kardialen JPH2-Knockdowns im Atrium wurden deshalb auf das 2p40mg-Protokoll festgelegt.

Gezüchtete Mäuse des C57BL/6J-Hintergrunds sind durch das Fehlen der mitochondrialen Transhydrogenase im Gegensatz zu C57BL/6N-Züchtungen vor oxidativem Stress und Herzinsuffizienz geschützt, weshalb ihre Verwendung in der Herzkreislaufforschung kontrovers diskutiert wird (Nickel et al. 2015). Da wir anders als van Oort et al. (2011) nur Mäuse des C57BL/6N-Hintergrunds verwendeten, entspricht unser JPH2-KnockdownProtokoll eher dem state of the art im Sinne von Nickel et al. (2015).

\subsubsection{Veränderungen von Proteinen im junktionalen Membrankomplex}

Per Western-Blot wurden Proteine untersucht, die 1) direkt Teil der CRU sind - der RyR2Kanal, dessen PKA-abhängig phosphorylierte Form RyR2-pS2808 und der spannungsabhängige LTCC Cav1.2 - sowie Proteine, die 2) funktionell für die zytosolische $\mathrm{Ca}^{2+}$ Homöostase von Bedeutung sind oder strukturell mit CRU in Verbindung stehen - insbesondere JPH2, SERCA2a, das SERCA2a-inhibierende Phosphoprotein PLN sowie das Membranprotein Cav3 (siehe 3.1.2).

\subsubsection{1 Änderungen der Proteinexpression in der Kalzium-Freisetzungseinheit:} Junctophilin-2, Cav1.2 und der Ryanodin-Rezeptor Typ 2

Die Reduktion der JPH2-Proteinexpression auf $43 \%$ bei unveränderter RyR2Proteinexpression führte $\mathrm{zu}$ einer stöchiometrisch bedingten Veränderung der JPH2/RyR2-Ratio in der Knockdown-Gruppe. Die Proteinexpression von RyR2-pS2808 und Cav1.2 nahm dabei nicht signifikant ab ( $\mathrm{p}=0,13$ bzw. 0,08), allerdings war das Proteinexpressionsverhältnis von RyR2-pS2808 zu RyR2 und von JPH2 zu Cav1.2 und RyR2 signifikant vermindert. Van Oort et al. (2011) beschrieben für den ventrikulären JPH2-Knockdown ebenfalls eine unveränderte RyR2- und Cav1.2-Proteinexpression, jedoch war das Verhält- 
nis von RyR2-pS2808 zu RyR2 unverändert. Dagegen wurden in einem Mausmodell druckinduzierter Hypertrophie vier Wochen nach TAC verminderte atriale Proteinexpressionswerte für RyR2 gefunden, dadurch war die Proteinexpression von RyR2-pS2808 im Verhältnis zu RyR2 gestiegen (Brandenburg et al. 2016a). Eine atriale JPH2-Abnahme wirkt sich demnach möglicherweise anders auf die Regulation von hochphosphoryliertem RyR2pS2808 aus als der ventrikuläre JPH2-Knockdown, vereinbar mit der Hypothese einer stark verminderten JPH2/RyR2-Stöchiometrie in den Atrien.

\subsubsection{2 Änderungen der Proteinexpression am sarkoplasmatischen Retikulum: SERCA2a und Phospholamban}

Murine Wildtyp-Atrien exprimieren mehr als doppelt so viel SERCA2a-Protein wie die Ventrikel, während die atriale PLN-Proteinexpression im Vergleich zum Ventrikel sehr gering ist (Brandenburg et al. 2016a). Der für die atriale Differenzierung wichtige Transkriptionsfaktor COUP-TFII supprimiert die Transkription von PLN in den Atrien, aber nicht den Ventrikeln (Wu et al. 2013). Die Inhibition von SERCA2a durch PLN ist daher im murinen Atrium von untergeordneter Bedeutung, während SERCA2a für die $\mathrm{Ca}^{2+}$ Aufnahme aus dem Zytosol ins SR eine große Rolle spielt (Brandenburg et al. 2018; Lüss et al. 1999; Walden et al. 2009). Interessanterweise war die Proteinexpression von SERCA2a nach JPH2-Knockdown im Atrium im Mittel sogar um 43 \% erhöht. Im Ventrikel war kein signifikanter SERCA2a-Anstieg nachweisbar (van Oort et al. 2011). Vier Wochen nach TAC wurden dagegen proteinbiochemisch signifikant verminderte atriale SERCA2a-Werte gemessen; in Verbindung mit hochreguliertem RyR2-pS2808 wurde daher die Möglichkeit einer zytosolischen $\mathrm{Ca}^{2+}$-Überladung und pro-arrhythmischer $\mathrm{Ca}^{2+}$-Wellen postuliert (Brandenburg et al. 2016a). Die PLN-Proteinexpression wurde weder im Atrium (siehe 3.1.2) noch im Ventrikel durch JPH2-Knockdown (van Oort et al. 2011) noch im Atrium durch TAC (Brandenburg et al. 2016a) signifikant verändert.

\subsubsection{3 Änderungen der Proteinexpression am Tubulus: Caveolin-3}

Das muskelspezifische integrale Membranprotein Cav3 befindet sich in Caveolae und ist als Marker für Membranstrukturen etabliert (Parton et al. 1997). Interessanterweise stieg nach JPH2-Knockdown die atriale Cav3-Proteinexpression im Mittel um 50\%. Dieses Ergebnis ging mit einer Proliferation von AT und einer erhöhten TAT-Netzwerkdichte einher (siehe 4.2.4 für weitere Erläuterung).

Zusammengefasst verursachte der herzspezifische JPH2-Knockdown proteinbiochemische Veränderungen im Atrium, die zu einer stöchiometrischen Entkopplung von JPH2 und RyR2, einer verminderten Phosphorylierung von RyR2-S2808 in der CRU und einer gesteigerten SERCA2a-Proteinexpression führten. Diese Ergebnisse legen eine von JPH2 abhängige differenzielle atriale $\mathrm{Ca}^{2+}$-Homöostase nahe. Zusätzlich ging die Zunahme von AT mit einer erhöhten Cav3-Proteinexpression in den Atrien einher. 


\subsection{Junctophilin-2-Knockdown induziert früh atriales Remodeling}

Krankheiten wie Herzinsuffizienz oder arterielle Hypertonie führen häufig zu pathologischem atrialen Remodeling mit kontraktiler Dysfunktion, myokardialer Hypertrophie, Dilatation, Fibrose, Entzündung, Vorhofflimmern, Blutstase, intraatrialer Thrombose und konsekutivem Hirn- oder Mesenterialinfarkt durch Thromboembolie (Schotten et al. 2011). Funktionelle Schäden wie kontraktile Dysfunktion und Vorhofflimmern können allerdings auch im strukturell gesunden Herzen auftreten, also ohne Nachweis eines histomorphologischen Korrelats (Brandenburg et al. 2016b). Als strukturelles Frühzeichen wurde in einem Hundemodell nach atrioventrikulärem overdrive pacing bereits nach zwei Tagen eine atriale Zellhypertrophie bei verminderter LVEF festgestellt, ohne dass die Atrien dilatiert oder fibrosiert waren (Ramadeen et al. 2012). Atriale Dysfunktion wird neuerdings als Kausalfaktor in der Entstehung von Herzinsuffizienz und Vorhofflimmern diskutiert (Goette et al. 2017), jedoch bleibt der Übergang vom gesunden zum hypertrophierten oder dilatierten, dysfunktionalen Atrium bisher mechanistisch unzureichend verstanden (Brandenburg et al. 2016b; Guichard und Nattel 2017).

\subsubsection{Atriale Dysfunktion bei akuter Herzinsuffizienz ohne strukturelles Korrelat}

Van Oort et al. (2011) injizierten eine Tamoxifen-Gesamtdosis von 150 mg/kg Körpergewicht über einen Zeitraum von fünf Tagen. Bereits eine Woche nach Abschluss der Behandlung lag die Überlebensrate in der Knockdown-Gruppe bei nur noch 60 \%, während kein Tier der Kontroll-Gruppe verstorben war. Untersuchungen der verbliebenen Tiere der Knockdown-Gruppe zeigten echokardiographisch eine signifikante Reduktion der LVEF und eine Zunahme des LVIDd, also eine verminderte Auswurfleistung bei Volumenbelastung des linken Ventrikels. Die linksventrikuläre Hinterwanddicke hatte sich indes nicht verändert, sodass hypertrophe Umbauprozesse als Ätiologie für die Entwicklung dieser akuten Herzinsuffizienz ausgeschlossen wurden (van Oort et al. 2011).

Im Gegensatz zu den ventrikulären Studien nutzte unser JPH2-Knockdown-Protokoll eine niedrigere Gesamtdosis (40 mg/kg Körpergewicht) und einen längeren Beobachtungszeitraum (zwei Wochen). Echokardiographisch wurden eine reduzierte Auswurfleistung und Kontraktilität in der JPH2-Knockdown-Gruppe gemessen: Die Werte für LVEF und LVFS waren signifikant vermindert; zusätzlich konnten wir auch im linken Atrium eine Reduktion des LAFS-Wertes nachweisen (siehe 3.2), obwohl die Proteinexpression von SERCA2a erhöht war. Interessanterweise wurden sowohl die Dilatation als auch die Hypertrophie der linken Herzhöhlen über die unveränderten Werte von LVIDd, LAIDd und AWThd ausgeschlossen. Im Mausmodell der druckinduzierten Hypertrophie zeigte sich die atriale Dysfunktion vier Wochen nach TAC ebenfalls in einer verminderten LAFS, allerdings waren diese Herzen auch strukturell im Sinne einer Dilatation und Hypertrophie geschädigt, denn LAIDd, AW'Thd und Herzgewicht waren erhöht, außerdem war die SERCA2aProteinexpression vermindert (Brandenburg et al. 2016a). Die Herzen unserer JPH2- 
Knockdown-Gruppe hatten zwar ein signifikant höheres Gewicht (absolut und in Relation zum Körpergewicht, gewogen direkt nach der Organentnahme) als diejenigen der KontrollGruppe (siehe 3.3). Dieser Wert ist jedoch stark davon abhängig, wie viel Blut sich noch im entnommenen Herzen befindet, und wird von unseren Daten aus Zellisolation (siehe 4.2.2) und Histologie (siehe 4.2.3) nicht gestützt. Zur zukünftigen genaueren Bewertung dieses Sachverhalts wäre es nötig, die Messung des Herzgewichts zum Beispiel über eine vorangehende Spülung zu standardisieren oder das Trockengewicht zu bestimmen.

Die auf $0 \%$ reduzierte Mortalität unseres JPH2-Knockdown-Protokolls (sowohl 2p40mg als auch 4p40mg) konnte zeigen, dass frühe funktionelle Veränderungen wie eingeschränkte Kontraktilität in Ventrikel und Atrium nicht zwingend mit einer Dilatation oder Hypertrophie im frühen Stadium korrelieren.

\subsubsection{Fehlende Hypertrophie isolierter atrialer Kardiomyozyten}

Zellhypertrophie ist häufig Bestandteil atrialen Remodelings (Schotten et al. 2011). Zahlreiche tierbezogene Interventionsmodelle zur Induktion der Herzinsuffizienz führen im Atrium und im Ventrikel zu myozytärer Hypertrophie, darunter die akute Druckbelastung des linken Herzens durch TAC und der MI durch permanente Ligatur des Ramus interventricularis anterior (Brandenburg et al. 2016a; Wagner et al. 2012). Zudem fand man in einem früheren JPH2-Knockdown-Modell hypertrophierte HL-1-Zellen (Landstrom et al. 2011). Van Oort et al. (2011) schlossen Hypertrophie als Ursache der Herzinsuffizienz nach JPH2-Knockdown dagegen sowohl echokardiographisch als auch histologisch und durch Isolation von VM aus.

Die von uns nach Isolation untersuchten lebenden AM unterschieden sich 2p40mg zwischen Kontroll- und Knockdown-Gruppe nicht in Bezug auf Länge, Breite oder deren Verhältnis und bewegten sich mit einer Größe von etwa $125 \mu \mathrm{m} \times 16 \mu \mathrm{m}$ in normalen Dimensionen (siehe 3.4.1). Es ist möglich, dass die Hypertrophie sich in unserem Modell erst innerhalb einer größeren Zeitspanne ausbildet; so lag der Untersuchungszeitpunkt der oben genannten Studien bei frühestens vier Wochen nach der Intervention (4pTAC bzw. 4pMI) oder mit bis zu acht Wochen (8pMI) sogar deutlich später (Brandenburg et al. 2016a; Wagner et al. 2012). Entsprechend fehlten eine Woche nach ventrikulärem JPH2Knockdown hypertrophe Umbauvorgänge (van Oort et al. 2011). Zudem ist bekannt, dass das TAC-Modell nur bedingte Vergleichbarkeit zur menschlichen Herzinsuffizienz bietet, da das plötzliche Auftreten eines transaortalen Druckgradienten von $80 \mathrm{mmHg}$ als Simulation vermehrter Nachlast einen sofortigen exogenen Stressor darstellt, während sich eine Aortenklappenstenose pathophysiologisch langsamer entwickelt (Patten und Hall-Porter 2009). Möglicherweise eröffnet unser JPH2-Knockdown-Modell Möglichkeiten, atriales Remodeling und die Entwicklung der Herzinsuffizienz über einen längeren Zeitraum zu studieren. 


\subsubsection{Histologischer Ausschluss atrialer Fibrose, Hypertrophie und Dilatation}

Reaktive Fibrose ist wie myozytäre Hypertrophie ein häufiger Bestandteil atrialen Remodelings, z. B. als Antwort auf starke mechanische Beanspruchung, bildet sich allerdings mit Verzögerung aus (Schotten et al. 2011). Hunde-Myozyten waren zwei Tage nach atrialem overdrive pacing zwar hypertrophiert, das umliegende Gewebe war jedoch nicht von Fibrose befallen (Ramadeen et al. 2012). Allerdings wurde vier Wochen nach TAC eine ausgeprägte Fibrose in murinen AM beobachtet (Brandenburg et al. 2019).

Histologische Anschnitte beider Atrien, des rechten Ventrikels und der Aorta auf Klappenebene, die mit HE- und Sirius-Red/Fast-Green-Färbungen behandelt wurden, zeigten weder in der Kontroll- noch in der Knockdown-Gruppe atriale Fibrose (siehe 3.5). Atriale Hypertrophie und Dilatation wurden ebenfalls ausgeschlossen, konsistent mit den Ergebnissen aus Echokardiographie und Zellisolation. Die Ursache der bestehenden atrialen Dysfunktion war damit weder im Gewebe noch auf äußerlich-struktureller Zellebene ersichtlich und erforderte Untersuchungen der myozytären Ultrastruktur.

\subsubsection{Reorganisation des TAT-Netzwerks}

\subsubsection{Bekannte Auswirkungen der Herzinsuffizienz auf das TAT-Netzwerk}

Herzinsuffizienz ist durch gravierende Umbauvorgänge innerhalb des TAT-Netzwerks gekennzeichnet. In einem Mausmodell von MI (VM) nahm vier Wochen nach Intervention die Dichte des TAT-Netzwerks dadurch zu, dass vor allem AT und OT proliferierten, während TT in absoluter Länge unverändert waren (Wagner et al. 2012). Interessanterweise war hier die JPH2-Expression proteinbiochemisch signifikant vermindert. Auch vier Wochen nach TAC (AM) stieg die Zahl von AT im Verhältnis zu TT signifikant (Brandenburg et al. 2016a). Van Oort et al. (2011) beschrieben die Veränderungen am TAT-Netzwerk durch JPH2-Knockdown (VM) als ,signifikante Störung mit Verlust an T-Tubulus-Strukturen“.

\subsubsection{Proliferation axialer Tubuli nach Junctophilin-2-Knockdown}

Wir untersuchten die Auswirkungen des JPH2-Knockdowns auf das TAT-Netzwerk lebender AM durch quantitative Analysen konfokalmikroskopischer Aufnahmen von AM, die mit dem lipophilen Membranfärbstoff di-8-ANEPPS behandelt worden waren (siehe 3.4.2). Konsistent mit obigen Studien wurde eine prozentuale Umverteilung der Ausrichtung bestimmter Komponenten im Sinne eines pathologischen Remodelings festgestellt: In der Knockdown-Gruppe nahmen AT zulasten von TT anteilig zu. Die absolute Dichte des TATNetzwerks stieg signifikant, da Komponenten aller Ausrichtungen (AT, OT und TT) proliferierten (konsistent mit erhöhter Cav3-Proteinexpression, siehe 4.1.2). Im Verhältnis zu TT war der Zuwachs von AT jedoch signifikant stärker. Im Hinblick auf die wichtige Rolle zentraler AT in AM als Plattform für hocheffiziente JMC stellte sich die Frage, wie ein deutlicher Zuwachs von AT mit atrialer Dysfunktion zusammenhängen kann, wenn doch die Super-hubs an AT seit neusten Ergebnissen das Fundament der schnellen atrialen 
elektromechanischen Kopplung darstellen (Brandenburg et al. 2016a). Die Bestandsaufnahme des TAT-Netzwerks allein bringt jedoch keinen Nachweis über den Zustand dort ansässiger CRU. Wir untersuchten daher die JMC auf subzellulärer Ebene mittels hochauflösender Immunfluoreszenz-Nanoskopie.

\subsubsection{Störung junktionaler Membrankomplexe an axialen Tubuli}

Konfokalmikroskopische und hochauflösende STED-Aufnahmen isolierter AM bestätigten frühere Studien, erweiterten das Verständnis von AM und zeigten die beginnende Störung von JMC zwei Wochen nach JPH2-Knockdown (siehe 3.6). In AM war eine differenzielle Verteilung von JPH2-Clustern offensichtlich. Analog zu RyR2 befanden sich große junktionale JPH2-Cluster in Assoziation mit AT im Zentrum der Zelle (markiert durch Cav3), während kleinere nicht-junktionale JPH2-Cluster (und RyR2-Cluster) entlang der Z-Linien sichtbar waren. Durch das JPH2-Knockdown-Protokoll verloren große zentrale junktionale AT-assoziierte JPH2-Cluster signifikeant an Größe. Insgesamt war die Morphologie der JPH2Cluster nach JPH2-Knockdown breiter gestreut, dadurch blieb ihr Flächenanteil unverändert. Dagegen verloren RyR2-Cluster sowohl an Größe als auch an Flächenanteil und waren zu einem geringeren Prozentsatz mit JPH2-Signalen kolokalisiert. Die Überlappungsfläche von RyR2 mit JPH2 nahm ebenfalls ab. Der JPH2-Knockdown sorgte also für eine fundamentale Störung der RyR2-Cluster. Funktionelle Konsequenz könnte eine Störung der $\mathrm{Ca}^{2+}-$ Homöostase und -Transienten und damit letztlich der Kontraktilität sein.

Wenn besonders zentrale junktionale JPH2-Cluster entlang AT durch den JPH2-Knockdown gestört werden, was durch Größenabnahme und Dispersion gemessen werden kann, und auch das RyR2-Cluster-Verhalten gestört ist, messbar durch Größenabnahme und geringere Überlappung mit JPH2, so kann daraus geschlossen werden, dass vor allem funktionell bedeutsame, hochphosphorylierte RyR2-pS2808 in Super-bubs entlang AT von dieser Störung betroffen sind, da sich niedrigphosphoryliertes RyR2 eher in transversalen Perlschnurketten entlang der Z-Linien befindet, keine Assoziation zum TAT-Netzwerk besitzt und ohnehin mit kleineren JPH2-Clustern kolokalisiert (Brandenburg et al. 2016a). Der biochemisch gemessene signifikant verminderte Anteil an RyR2-S2808-Phosphorylierung unterstützt diese Hypothese. In Studien des ventrikulären JPH2-Knockdowns zeigte sich ebenfalls eine geringere Überlappung von RyR2 mit JPH2, wobei die Größe der RyR2Cluster unverändert war (Munro et al. 2016), außerdem war auch die Kolokalisation von RyR2 mit Cav1.2 gestört (van Oort et al. 2011). Aus einem Rattenmodell ist bekannt, dass RyR2 in VM im Rahmen einer TAC-induzierten Herzinsuffizienz durch Umbauprozesse am TAT-Netzwerk von zuvor scheinbar kolokalisierten Cav1.2-Kanälen „verlassen“ werden (Song et al. 2006), was sich negativ auf die elektromechanische Kopplung auswirkt.

Spannungsgesteuerte Cav1.2-Cluster an AT, die im atrialen Super-bub-Modell den funktionell wichtigen Gegenpart zum RyR2-pS2808 stellen (Brandenburg et al. 2016a), verloren durch den JPH2-Knockdown ebenfalls an Größe. Dagegen waren viele kleinere Cav1.2-Cluster diffu- 
ser im Zytosol verteilt und nicht mehr mit dem atrialen TAT-Netzwerk assoziiert. Zur Regulation der elektrischen Eigenschaften der Zellmembran in AM können bestimmte Kaliumkanäle im Golgi-Apparat zurückgehalten und bei vermehrtem Stress vesikulär ins Sarkolemm fusioniert werden (Arakel et al. 2014). Ein solcher Mechanismus wäre auch für Cav1.2 denkbar, sodass das diffuse Cav1.2-Signal nach JPH2-Knockdown möglicherweise sarkolemmferne Cluster erfasst, die sich in gespeicherter Form auf dem sekretorischen Pfad in Vesikeln oder im Golgi-Apparat befinden.

In der Zusammenschau proliferierten das TAT-Netzwerk und insbesondere AT zwar nach dem JPH2-Knockdown, allerdings bildeten sich entlang dieser Tubuli gestörte JMC aus, die morphologisch durch kleinere JPH2- und RyR2-Cluster und räumliche Dissoziation von JPH2, Cav1.2 und RyR2-pS2808 gekennzeichnet waren.

\subsection{Differenzielle Verteilung von Junctophilin-2 in atrialen Myozyten}

In aus Wildtypmäusen isolierten AM war eine große Menge der RyR2-Kanäle - typischerweise in transversalen Cluster-Ketten entlang der Z-Linien angeordnet - nicht-junktional, niedrigphosphoryliert und von kleineren Mengen JPH2 stabilisiert als junktionales RyR2pS2808 an AT. Daraus ergibt sich die Hypothese, dass die subzellulär lokal unterschiedlich exprimierten Gruppen von junktionalem und nicht-junktionalem JPH2 auch unterschiedliche Aufgaben übernehmen: 1) Große JPH2-Cluster könnten strukturell für die stabile Bindung großvolumiger AT an das SR notwendig sein, da eine hohe lokale Konzentration ihrer MORN-Domänen zahlreiche Affinitäten für nichtkovalente Bindungen mit geladenen Phospholipiden akkumuliert (Takeshima et al. 2000). 2) Kleinere JPH2-Cluster könnten ausreichend sein für die funktionelle Stabilisierung der RyR2 im Sinne der Regulation ihrer $\mathrm{Ca}^{2+}$-Kanalfunktion abseits von Junktionen.

\subsection{Verzögerung der atrialen Erregungsausbreitung im EKG}

Atriales Remodeling beinhaltet neben strukturellen Veränderungen auch eine mögliche Störung der Funktion des Sinusknotens und der Erregungsleitung sowie eine veränderte Refraktäritätszeit (Sanders et al. 2003). Die Prognose der chronischen Herzinsuffizienz wird durch Erregungsleitungsstörungen wie AV-Block negativ beeinflusst (Schoeller et al. 1993). Die Ursachen und Mechanismen dieser Erregungsleitungsstörungen sind Gegenstand aktueller Forschung. So wurden beispielsweise Veränderungen der Connexine (Cx) mit Vorhofflimmern und verlangsamter Erregungsleitung in Mensch und Tier in Verbindung gebracht (Kato et al. 2012). Die Messung des PR-Intervalls im EKG kann in diesem Zusammenhang die atrioventrikuläre Erregungsausbreitung abschätzen (Noujaim et al. 2004).

Atriale Dilatation sorgt für eine längere Strecke vom Sinus- zum AV-Knoten, vor allem werden aber Veränderungen der atrialen Proteinexpression für verlängerte PR-Intervalle verantwortlich gemacht (Nikolaidou et al. 2015). Acht Wochen nach Induktion eines links- 
ventrikulären MI im Hasen fand man neben atrialer Dilatation eine um 7 ms (10\%) verlängerte PQ-Zeit bei fibrotischem AV-Knoten und Veränderungen der Expression der Gapjunction-Proteine $\mathrm{Cx} 40$ und -43 (Nisbet et al. 2016). In einem früheren Hypertrophiemodell der Herzinsuffizienz im Hasen wurde die verlängerte PR-Zeit durch die verminderte Expression des hauptsächlich durch cAMP regulierten Kationenkanals Typ 1 (HCN1, hyperpolarization-actived cyclic nucleotide-gated channel type 1) sowie von Cav1.3, Cx40 und Cx43 bei vergrößerten Herzhöhlen erklärt (Larsson 2010; Nikolaidou et al. 2015).

Die Untersuchung der JPH2-Knockdown-Gruppe ergab BASAL verlängerte (normalisierte) Zeiten für die P-Welle, das PQ- und das PR-Intervall im Vergleich zur Kontroll-Gruppe (siehe 3.7). Die Normalisierung aller Daten auf die mittlere Herzfrequenz der Kontrollgruppe war wegen der signifikant erhöhten Herzfrequenz der Knockdown-Gruppe notwendig. Durch positiv dromotrope $\beta$-adrenerge Stimulation nach Isoproterenol-Injektion nivellierten sich die Unterschiede in Herzfrequenz und P-Welle, allerdings war die atrioventrikuläre Überleitung - erkennbar am längeren PQ- und PR-Intervall - in der Knockdown-Gruppe weiterhin verlangsamt. Bei einer Maus der Knockdown-Gruppe fand sich BASAL intermittierend ein junktionaler Ersatzrhythmus, also eine vom AV-Knoten oder His-Bündel ausgehende Erregung bei fehlender P-Wellen-Überleitung. Atriale Dilatation und Fibrose wurden als Ursache für diese elektrophysiologischen Störungen ausgeschlossen, wobei eine direkte histologische und proteinbiochemische Untersuchung des AVKnotens nicht Gegenstand dieser Arbeit war.

Zur genaueren mechanistischen Charakterisierung der elektrophysiologischen Auswirkungen des JPH2-Knockdowns wäre es also zunächst notwendig, den Sinus- und AV-Knoten auf Veränderungen zu untersuchen (z. B. Hinweise auf lokale Fibrose oder Veränderungen der HCN1-Expression). Weiterhin könnte eine Untersuchung der gap junctions (über Cx40 und Cx43) Erklärungen bieten, ebenso wie die Untersuchung membranständiger NatriumKanäle und -Transporter wie der SCN1-Familie (spannungsabhängiger $\mathrm{Na}^{+}$-Kanal Typ 1) und NCX. Kürzlich konnte in Rattenherzen gezeigt werden, dass die Substanz DTBHA (3,5-Di-tert-butyl-4-hydroxyanisol), die an RyR2 bindet und als Antioxidans und LTCCBlocker bekannt ist, die PQ-Zeit verlängern kann (Pessina et al. 2018). Demnach kann die atrioventrikuläre Überleitung auch durch (hier medikamentöse) Manipulation der CRU beeinflusst werden, auch wenn eine mechanistische Erklärung fehlt (Pessina et al. 2018).

Die Modulation des atrialen Aktionspotentials stellt einen multifaktoriellen Prozess dar, der von Ionenkanälen und damit von elektrischer und metabolischer Gewebsaktivität abhängt (Brandenburg et al. 2016b). Es bleibt festzuhalten, dass die im EKG gemessene atrioventrikuläre Überleitung nach JPH2-Knockdown einschränkt war. Zusammen mit den Ergebnissen der Echokardiographie belegt dies funktionelle Störungen in vivo, die nicht durch atriale Hypertrophie, Fibrose oder Dilatation erklärt werden können. 


\section{$5 \quad$ Zusammenfassung und Ausblick}

In dieser Arbeit wurden die Auswirkungen des herzspezifischen JPH2-Knockdowns auf Struktur und Funktion der Atrien und AM untersucht. Die atriale JPH2-Proteinexpression betrug im Western-Blot etwa $20 \%$ verglichen mit dem Ventrikel. Adulte transgene Mäuse der Linie MCM-shJPH2 zeigten nach einmaliger i.p.-Injektion von $40 \mathrm{mg} / \mathrm{kg}$ Körpergewicht Tamoxifen ein atriales JPH2-Proteinexpressionsniveau von etwa $42 \%$ im Vergleich zu MCM-Mäusen als Kontrolle. Alle Tiere überlebten die zwei Wochen dauernde Beobachtungsphase. Proteinbiochemisch war das Verhältnis der Expression von JPH2 zu RyR2 signifikant vermindert, ebenso wie die Expression von PKA-abhängig phosphoryliertem RyR2-pS2808. Die RyR2-Expression selbst war unverändert. Die Expression von SERCA2a und Cav3 war signifikant erhöht. Echokardiographisch führte der JPH2Knockdown $\mathrm{zu}$ atrialem und ventrikulärem Funktionsverlust bei strukturell (histologisch) unveränderten Herzen, nämlich Abwesenheit von Dilatation, Hypertrophie und Fibrose. Isolierte AM waren ebenfalls nicht hypertrophiert, zeigten aber eine starke Proliferation des TAT-Netzwerks, insbesondere der AT. In Immunfluoreszenzuntersuchungen fixierter AM zeigte sich eine bisher unbekannte differenzielle Verteilung von JPH2 in große junktionale und kleine nichtjunktionale Cluster. Junktionales JPH2 war mit AT assoziiert. Die ClusterMorphologie junktionaler PKA-hochphosphorylierter RyR2-Kanäle entlang AT war nach JPH2-Knockdown gestört, ebenso waren TAT-assoziierte spannungsabhängige Cav1.2-Cluster verkleinert. Elektrokardiographisch war die atrioventrikuläre Erregungsüberleitung nach JPH2-Knockdown verlangsamt. Diese Ergebnisse veranschaulichen, dass der JPH2Knockdown zu atrialer Dysfunktion und pathologischem Remodeling führte, jedoch ohne jegliche Mortalität (bis zu vier Wochen nach Tamoxifenbehandlung), atriale Hypertrophie, Dilatation oder Fibrose. Unsere Daten belegen zusammenfassend eine molekulare Störung atrialer JMC nach herzspezifischem JPH2-Knockdown.

Auf Basis der Ergebnisse dieser Arbeit wurden zum Verständnis der Auswirkungen der JPH2-Defizienz auf die Funktion dieser gestörten JMC und damit auf die atriale elektromechanische Kopplung funktionelle $\mathrm{Ca}^{2+}$-Messungen durchgeführt $\left(\mathrm{Ca}^{2+}\right.$-Transienten und -Sparks). Zusätzlich wurden im Umkehrschluss die Effekte konstitutiver kardialer Überexpression von JPH2 im Atrium analysiert, besonders im Zusammenhang mit druckinduzierter Hypertrophie durch TAC. Außerdem wurden die kombinierten Auswirkungen des JPH2-Knockdowns und der TAC-Intervention auf murine Atrien untersucht. Die gewonnenen Ergebnisse wurden gemeinsam mit den Ergebnissen dieser Arbeit in der Zeitschrift JCI insight veröffentlicht (Brandenburg et al. 2019).

Unser Protokoll des JPH2-Knockdowns wurde entwickelt, um die hohe Mortalität (van Oort et al. 2011) durch akute Herzinsuffizienz zu verhindern. Obwohl der $\alpha$-MHC-Promoter für ventrikuläre Transgen-Studien entwickelt wurde, ist er auch im Atrium wirksam und war 
die Grundlage für den hier angewandten kardialen JPH2-Knockdown. Allerdings hat die hier gezeigte beginnende ventrikuläre Insuffizienz zwingenderweise Auswirkungen auf die Integrität des vorgeschalteten Atriums. In diesem Zusammenhang könnte die Verwendung des kürzlich vorgestellten atrienspezifischen Gentransfers über einen adeno-assoziierten viralen Vektor (AAV9) störende Variablen vermindern und so langfristig eine spezifische Behandlung der atrialen Dysfunktion in präklinischen Studien ermöglichen (Ni et al. 2019). Nichtsdestoweniger wird es für das klinische Verständnis wichtig sein, kombinierte atrioventrikuläre Phänotypen zu antizipieren und klinische Verlaufsformen der Herzinsuffizienz zu differenzieren.

Die Entdeckung der zellspezifischen differenziellen Distribution junktionaler und nichtjunktionaler JPH2-Cluster in AM bedarf weiterer Analysen in Bezug auf die Abnahme des JPH2-Proteins bei Herzinsuffizienz oder atrialen Rhythmusstörungen. Die Überexpression von JPH2 könnte für künftige therapeutische Konzepte bei atrialer Dysfunktion entsprechend ein zielführender Ansatz sein. 


\section{$6 \quad$ Anhang}

\subsection{Chemikalien}

\begin{tabular}{|c|c|c|}
\hline Substanz & Firma & Bestellnummer \\
\hline 2-Mercaptoethanol & Sigma & M3148 \\
\hline Acrylamid (Rotiphorese ${ }^{\circledR}$ Gel 30) & Roth & 3029.1 \\
\hline \multirow[t]{2}{*}{ BCS (bovines Kälberserum) } & Thermo Fisher & SH30073 \\
\hline & Scientific & \\
\hline BDM (2,3-Butandionmonoxim) & Sigma-Aldrich & B0753 \\
\hline BSA (bovines Serumalbumin) & Sigma-Aldrich & A8806 \\
\hline $\mathrm{CaCl}_{2} \times 2 \mathrm{H}_{2} \mathrm{O}$ & Merck & 17257 \\
\hline EGTA & Sigma-Aldrich & E4378 \\
\hline Essigsäure & Merck & 1000632500 \\
\hline Glukose & Sigma-Aldrich & G7021 \\
\hline Glyzerin & Sigma-Aldrich & G8773 \\
\hline Glyzin & Roth & 3908.3 \\
\hline $\mathrm{HCl} 37 \%$ & Sigma-Aldrich & 84422 \\
\hline HEPES & Sigma-Aldrich & $\mathrm{H} 4034$ \\
\hline $\mathrm{KCl}$ & Sigma-Aldrich & P5405 \\
\hline $\mathrm{KH}_{2} \mathrm{PO}_{4}$ & Merck & 4873 \\
\hline $\mathrm{KHCO}_{3}$ & Sigma-Aldrich & P7682 \\
\hline Kollagenase Typ II & Worthington & LS004177 \\
\hline Laminin & Sigma-Aldrich & L2020 \\
\hline Methanol & Roth & HN41.2 \\
\hline $\mathrm{MgSO}_{4} \times 7 \mathrm{H}_{2} \mathrm{O}$ & Sigma-Aldrich & M2773 \\
\hline Milchpulver & Roth & $\mathrm{T} 145.1$ \\
\hline $\mathrm{Na}_{2} \mathrm{HPO}_{4} \times 2 \mathrm{H}_{2} \mathrm{O}$ & Sigma-Aldrich & 71643 \\
\hline $\mathrm{NaCl}$ & Sigma-Aldrich & S5886 \\
\hline $\mathrm{NaHCO}_{3}$ & Sigma-Aldrich & S5761 \\
\hline $\mathrm{NaOH}$ & Roth & 6771.3 \\
\hline
\end{tabular}




\begin{tabular}{lll}
\hline \hline Substanz & Firma & Bestellnummer \\
\hline PBS (phosphatgepufferte Salzlösung) & Gibco & 10010 \\
PFA (Paraformaldehyd) & Sigma-Aldrich & 158127 \\
PhosSTOPTM (Phosphatase-Inhibitor) & Roche & 04906837001 \\
Ponceau S & Sigma-Aldrich & P3504 \\
Protease-Inhibitor-Cocktail (cOmpleteTM) & Roche & 11872580001 \\
Proteinmarker V & Peqlab & $27-2211$ \\
Roti®-Histofix 4\% & Roth & P087 \\
Roticlear ${ }^{\circledR}$ & Roth & A538.5 \\
Saccharose & Sigma-Aldrich & S0389 \\
SDS (20\% Natriumdodezylsulfat-Lösung) & Fluka & 05030 \\
Sojabohnenöl & Sigma-Aldrich & S7381 \\
Taurin & Sigma-Aldrich & T8691 \\
TEMED (N,N,N`N“-Tetramethylethylendiamin) & Sigma-Aldrich & T9281 \\
TRIS (Tris(hydroxymethyl)aminomethan) & Roth & 4855.3 \\
Triton X-100 & Sigma-Aldrich & T9284 \\
Tween ${ }^{\circledR 20}$ & Sigma-Aldrich & P1379 \\
\hline \hline
\end{tabular}

\subsection{Medikamente}

\begin{tabular}{lll}
\hline Substanz & Firma & Bestellnummer \\
\hline Isoproterenol & Sigma-Aldrich & I5627 \\
Tamoxifen & Sigma-Aldrich & T5648-1g \\
\hline \hline
\end{tabular}

\subsection{Färbstoffe und Einbettmedien}

\begin{tabular}{lll}
\hline \hline Substanz & Firma & Bestellnummer \\
\hline di-8-ANEPPS & $\begin{array}{l}\text { Thermo Fisher } \\
\text { Scientific }\end{array}$ & D-3167 \\
Direct Red 80 & Sigma-Aldrich & $365548-5 \mathrm{~g}$ \\
Eosin-Y-Lösung & Sigma-Aldrich & $118 \mathrm{~K} 4355$ \\
Fast Green FCF & Roth & $0301.1-25 \mathrm{~g}$ \\
Hämatoxylinlösung (Mayer's) & Sigma-Aldrich & $039 \mathrm{~K} 4367$ \\
ProLong® Gold Antifade mit DAPI & Invitrogen & P36934 \\
\hline
\end{tabular}




\begin{tabular}{lll}
\hline \hline Substanz & Firma & Bestellnummer \\
\hline Roti ${ }^{\text {R-Histokitt }}$ & Roth & 6638.1 \\
\hline \hline
\end{tabular}

\subsection{Antikörper}

\subsubsection{Primärantikörper für Western-Blots}

\begin{tabular}{llll}
\hline \hline Antikörper & Verdünnung & Firma & Bestellnummer \\
\hline anti-Cav1.2 (rabbit) & $1: 200$ & abcam & ab58552 \\
anti-Cav3 (rabbit) & $1: 1.000$ & abcam & ab2912 \\
anti-GAPDH (mouse) & $1: 160.000$ & Biotrend & 5G4 Mab 6C5 \\
anti-JPH2 (rabbit) & $1: 1.000$ & Invitrogen antibodies & 40-5300 \\
anti-PLN (mouse) & $1: 2.500$ & Abberior & ab2865 \\
anti-RyR2 Prestige $($ rabbit) & $1: 2.500$ & Sigma-Aldrich & HPA020028 \\
anti-RyR2 pS2808 (rabbit) & $1: 1.000$ & Badrilla & A010-30 \\
anti-SERCA2a (rabbit) & $1: 1.000$ & Badrilla & A010-20 \\
\hline \hline
\end{tabular}

\subsubsection{Sekundärantikörper für Western-Blots}

\begin{tabular}{llll}
\hline \hline Antikörper & Verdünnung & Firma & Bestellnummer \\
\hline $\begin{array}{c}\text { IRDye }(\text { 680RD } \\
\quad \text { donkey anti-mouse })\end{array}$ & $1: 13.000$ & LI-COR Biosciences & $926-68072$ \\
$\begin{array}{c}\text { IRDye }{ }^{\circledR} \text { 680RD } \\
\quad \text { donkey anti-rabbit })\end{array}$ & $1: 13.000$ & LI-COR Biosciences & $925-68073$ \\
$\begin{array}{c}\text { IRDye }{ }^{\circledR} 800 \mathrm{CW} \\
(\text { donkey anti-mouse })\end{array}$ & $1: 13.000$ & LI-COR Biosciences & $935-32212$ \\
$\begin{array}{c}\text { IRDye }(\text { 800CW } \\
(\text { donkey anti-rabbit })\end{array}$ & $1: 13.000$ & LI-COR Biosciences & $926-32213$ \\
\hline \hline
\end{tabular}

\subsubsection{Primärantikörper für konfokale und STED-Mikroskopie}

\begin{tabular}{llll}
\hline \hline Antikörper & Verdünnung & Firma & Bestellnummer \\
\hline anti-Cav3 (mouse) & $1: 500$ & BD Biosciences & 610421 \\
anti-Cav1.2 (rabbit) & $1: 500$ & Alomone labs & ACC-003 \\
anti-JPH2 (rabbit) & $1: 500$ & Invitrogen antibodies & $40-5300$ \\
anti-RyR2 (mouse) & $1: 500$ & Invitrogen antibodies & MA3-916 \\
\hline
\end{tabular}




\begin{tabular}{llll}
\hline \hline Antikörper & Verdünnung & Firma & Bestellnummer \\
\hline anti-RyR2-pS2808 (rabbit) & $1: 500$ & Badrilla & A010-30 \\
\hline \hline
\end{tabular}

\subsubsection{Sekundärantikörper für konfokale und STED-Mikroskopie}

\begin{tabular}{|c|c|c|c|}
\hline Antikörper & Verdünnung & Firma & Bestellnummer \\
\hline $\begin{array}{l}\text { Alexa Fluor }{ }^{\mathrm{TM}} 514 \\
\text { (goat anti-mouse) }\end{array}$ & $1: 1.000$ & $\begin{array}{l}\text { Thermo Fisher } \\
\text { Scientific }\end{array}$ & A-31555 \\
\hline $\begin{array}{l}\text { Alexa Fluor }{ }^{\mathrm{TM}} 514 \\
\text { (goat anti-rabbit) }\end{array}$ & $1: 1.000$ & $\begin{array}{l}\text { Thermo Fisher } \\
\text { Scientific }\end{array}$ & A-31558 \\
\hline $\begin{array}{l}\text { Alexa Fluor }{ }^{\mathrm{TM}} 568 \\
\qquad \text { (goat anti-mouse) }\end{array}$ & $1: 1.000$ & $\begin{array}{l}\text { Thermo Fisher } \\
\text { Scientific }\end{array}$ & A-11031 \\
\hline $\begin{array}{l}\text { Alexa Fluor }{ }^{\mathrm{TM}} 633 \\
\text { (goat anti-rabbit) }\end{array}$ & $1: 1.000$ & $\begin{array}{l}\text { Thermo Fisher } \\
\text { Scientific }\end{array}$ & A-21070 \\
\hline $\begin{array}{l}\text { STAR } 488 \\
\quad \text { (goat anti-mouse) }\end{array}$ & $1: 1.000$ & Abberior & 2-0002-006-8 \\
\hline $\begin{array}{l}\text { STAR 635P } \\
\text { (goat anti-rabbit) }\end{array}$ & $1: 1.000$ & Abberior & $2-0012-007-2$ \\
\hline $\begin{array}{c}\text { Zenon }^{\mathrm{TM}} \text { Alexa Fluor } \\
\text { (rabbit Ig-G labeling kit } 647\end{array}$ & $1: 1.000$ & $\begin{array}{l}\text { Thermo Fisher } \\
\text { Scientific }\end{array}$ & Z25308 \\
\hline
\end{tabular}

\subsection{Geräte}

\begin{tabular}{|c|c|c|}
\hline Gerät & Anwendungsbereich & Firma \\
\hline Axiocam 105 Color & Histologie & ZEISS \\
\hline EKG-Elektroden (Maus) & Elektrokardiographie & emka TECHNOLOGIES \\
\hline inoLab® & pH-Messung & W'TW (Xylem Analytics) \\
\hline Kodak Image Station 4000R PRO & Western-Blot & Carestream Health Inc. \\
\hline $\begin{array}{l}\text { LSM710 } \\
\qquad \text { Plan-Apochromat 63x/1.4 NA } \\
\text { Öl-Objektiv }\end{array}$ & Konfokalmikroskopie & ZEISS \\
\hline MICCRA D-1 & $\begin{array}{l}\text { Western-Blot } \\
\qquad \text { (Dispergiergerät) }\end{array}$ & MICCRA GmbH \\
\hline Microplate-Spektrophotometer & $\begin{array}{l}\text { Western-Blot } \\
\qquad \text { (Photometrie) }\end{array}$ & BioTek \\
\hline $\begin{array}{l}\text { MicroScan }{ }^{\mathrm{TM}} \text { Transducer MS400 } \\
\text { für Vevo }{ }^{\circledR} 2100\end{array}$ & $\begin{array}{l}\text { Echokardiographie } \\
\qquad \text { (30-MHz-Schallkopf) }\end{array}$ & FUJIFILM VisualSonics \\
\hline
\end{tabular}




\begin{tabular}{lll}
\hline \hline Gerät & Anwendungsbereich & Firma \\
\hline Minivent 845 & $\begin{array}{c}\text { Echokardiograhie } \\
\text { (Beatmungsgerät) }\end{array}$ & Harvard Apparatus \\
Nasser Blot-Apparat & Western-Blot & Thermo Fisher Scientific \\
Odyssey® Fcx-Kamera & Western-Blot & LI-COR Biosciences \\
POC-R2 Messkammer & Konfokalmikroskopie & PeCon GmbH \\
TCS SP8 STED & STED-Mikroskopie & Leica Microsystems \\
HC PL APO CS2 100x/1.4 & & \\
Ö1-Objektiv & & \\
Vevo ${ }^{\circledR} 2100$ & Echokardiographie & FUJIFILM VisualSonics \\
\hline \hline
\end{tabular}

\subsection{Software}

\begin{tabular}{|c|c|c|}
\hline Programm & Anwendungsbereich & Anbieter \\
\hline Application Suite X & STED-Mikroskopie & Leica Microsystems \\
\hline ecgAUTO 3.3.5.10 & Elektrokardiographie & emka TECHNOLOGIES \\
\hline Fiji & Bildbearbeitung und -analyse & https://fiji.sc/ \\
\hline Image Studio ${ }^{\text {TM }}$ Software & Proteindetektion Western-Blot & LI-COR Biosciences \\
\hline IOX 2.10.0.40 & Elektrokardiographie & emka TECHNOLOGIES \\
\hline KC4TM & Photometrie & BioTek \\
\hline Molecular Imaging Software V5.0.3 & Proteindetektion Western-Blot & Carestream Health Inc. \\
\hline Office 2016 & Text- und Datenbearbeitung & Microsoft Corporation \\
\hline ZEN 2009 & Konfokalmikroskopie LSM710 & ZEISS \\
\hline \multirow[t]{7}{*}{ Zotero } & Literaturverwaltung & https://zotero.org/ \\
\hline & & Corporation for Digital \\
\hline & & Scholarship/ \\
\hline & & Roy Rosenzweig Center \\
\hline & & for History and New \\
\hline & & Media, George Mason \\
\hline & & University, VA, USA \\
\hline
\end{tabular}




\section{Literaturverzeichnis}

Arakel EC, Brandenburg S, Uchida K, Zhang H, Lin Y-W, Kohl T, Schrul B, Sulkin MS, Efimov IR, Nichols CG (2014): Tuning the electrical properties of the heart by differential trafficking of KATP ion channel complexes. J Cell Sci 127, 2106-2119

Beavers DL, Wang W, Ather S, Voigt N, Garbino A, Dixit SS, Landstrom AP, Li N, Wang Q, Olivotto I (2013): Mutation E169K in Junctophilin-2 Causes Atrial Fibrillation Due to Impaired RyR2 Stabilization. J Am Coll Cardiol 62, 2010-2019

Beavers DL, Landstrom AP, Chiang DY, Wehrens XHT (2014): Emerging roles of junctophilin-2 in the heart and implications for cardiac diseases. Cardiovasc Res 103, 198-205

Benjamin EJ, Virani SS, Callaway CW, Chamberlain AM, Chang AR, Cheng S, Chiuve SE, Cushman M, Delling FN, Deo R (2018): Heart Disease and Stroke Statistics-2018 Update: A Report From the American Heart Association. Circulation 137, e67-e492

Bers DM (2002): Cardiac excitation-contraction coupling. Nature 415, 198-205

Bootman MD, Higazi DR, Coombes S, Roderick HL (2006): Calcium signalling during excitation-contraction coupling in mammalian atrial myocytes. J Cell Sci 119, 3915-3925

Brandenburg S (2017): Über die differentielle Regulation von Ionenkanälen in spezifischen Nanodomänen atrialer und ventrikulärer Kardiomyozyten. Med Diss Göttingen 2017

Brandenburg S, Kohl T, Williams GSB, Gusev K, Wagner E, Rog-Zielinska EA, Hebisch E, Dura M, Didié M, Gotthardt M (2016a): Axial tubule junctions control rapid calcium signaling in atria. J Clin Invest 126, 3999-4015

Brandenburg S, Arakel EC, Schwappach B, Lehnart SE (2016b): The molecular and functional identities of atrial cardiomyocytes in health and disease. Biochim Biophys Acta 1863 , 1882-1893

Brandenburg S, Pawlowitz J, Fakuade FE, Kownatzki-Danger D, Kohl T, Mitronova GY, Scardigli M, Neef J, Schmidt C, Wiedmann F (2018): Axial Tubule Junctions Activate Atrial Ca2+ Release Across Species. Front Physiol 9, 1227

Brandenburg S, Pawlowitz J, Eikenbusch B, Peper J, Kohl T, Mitronova GY, Sossalla S, Hasenfuss G, Wehrens XH, Kohl P (2019): Junctophilin-2 expression rescues atrial dysfunction through polyadic junctional membrane complex biogenesis. JCI Insight $\underline{4}$

Brodde O-E, Bruck H, Leineweber K (2006): Cardiac adrenoceptors: physiological and pathophysiological relevance. J Pharmacol Sci 100, 323-337 
Carl SL, Felix K, Caswell AH, Brandt NR, Ball WJ, Vaghy PL, Meissner G, Ferguson DG (1995): Immunolocalization of sarcolemmal dihydropyridine receptor and sarcoplasmic reticular triadin and ryanodine receptor in rabbit ventricle and atrium. J Cell Biol 129, 673682

Chen B, Guo A, Zhang C, Chen R, Zhu Y, Hong J, Kutschke W, Zimmerman K, Weiss RM, Zingman L (2013): Critical roles of junctophilin-2 in T-tubule and excitationcontraction coupling maturation during postnatal development. Cardiovasc Res 100, 54-62

Dibb KM, Clarke JD, Horn MA, Richards MA, Graham HK, Eisner DA, Trafford AW (2009): Characterization of an extensive transverse tubular network in sheep atrial myocytes and its depletion in heart failure. Circ Heart Fail 2, 482-489

Dibb KM, Clarke JD, Eisner DA, Richards MA, Trafford AW (2013): A functional role for transverse (t-) tubules in the atria. J Mol Cell Cardiol $\underline{58}, 84-91$

Forbes MS, van Neil EE (1988): Membrane systems of guinea pig myocardium: ultrastructure and morphometric studies. Anat Rec 222, 362-379

Franzini-Armstrong C, Protasi F, Ramesh V (1999): Shape, size, and distribution of $\mathrm{Ca}(2+)$ release units and couplons in skeletal and cardiac muscles. Biophys J 77, 1528-1539

Garbino A, van Oort RJ, Dixit SS, Landstrom AP, Ackerman MJ, Wehrens XHT (2009): Molecular evolution of the junctophilin gene family. Physiol Genomics $\underline{37}, 175-186$

Goette A, Kalman JM, Aguinaga L, Akar J, Cabrera JA, Chen SA, Chugh SS, Corradi D, D'Avila A, Dobrev D (2017): EHRA/HRS/APHRS/SOLAECE expert consensus on atrial cardiomyopathies: Definition, characterization, and clinical implication. Heart Rhythm $\underline{14}, \mathrm{e} 3-\mathrm{e} 40$

Göktepe S, Abilez OJ, Parker KK, Kuhl E (2010): A multiscale model for eccentric and concentric cardiac growth through sarcomerogenesis. J Theor Biol 265, 433-442

Greiser M, Lederer WJ, Schotten U (2011): Alterations of atrial $\mathrm{Ca}(2+)$ handling as cause and consequence of atrial fibrillation. Cardiovasc Res $\underline{89}, 722-733$

Guichard J-B, Nattel S (2017): Atrial Cardiomyopathy: A Useful Notion in Cardiac Disease Management or a Passing Fad? J Am Coll Cardiol 0ㅡ, 756-765

Guo A, Hall D, Zhang C, Peng T, Miller JD, Kutschke W, Grueter CE, Johnson FL, Lin RZ, Song L-S (2015): Molecular Determinants of Calpain-dependent Cleavage of Junctophilin-2 Protein in Cardiomyocytes. J Biol Chem 290, 17946-17955

Guo A, Wang Y, Chen B, Wang Y, Yuan J, Zhang L, Hall D, Wu J, Shi Y, Zhu Q (2018): E-C coupling structural protein junctophilin-2 encodes a stress-adaptive transcription regulator. Science $\underline{362}$ 
Heidenreich PA, Trogdon JG, Khavjou OA, Butler J, Dracup K, Ezekowitz MD, Finkelstein EA, Hong Y, Johnston SC, Khera A (2011): Forecasting the future of cardiovascular disease in the United States: a policy statement from the American Heart Association. Circulation $\underline{123}, 933-944$

Ho KK, Pinsky JL, Kannel WB, Levy D (1993): The epidemiology of heart failure: the Framingham Study. J Am Coll Cardiol 22, 6A-13A

Kato T, Iwasaki Y, Nattel S (2012): Connexins and atrial fibrillation: filling in the gaps. Circulation $\underline{125}$, 203-206

Kirk MM, Izu LT, Chen-Izu Y, McCulle SL, Wier WG, Balke CW, Shorofsky SR (2003):

Role of the Transverse-Axial Tubule System in Generating Calcium Sparks and Calcium Transients in Rat Atrial Myocytes. J Physiol 547, 441-451

Kockskämper J, Sheehan KA, Bare DJ, Lipsius SL, Mignery GA, Blatter LA (2001): Activation and Propagation of $\mathrm{Ca} 2+$ Release during Excitation-Contraction Coupling in Atrial Myocytes. Biophys J $\underline{81}$, 2590-2605

Kotecha D, Holmes J, Krum H, Altman DG, Manzano L, Cleland JGF, Lip GYH, Coats AJS, Andersson B, Kirchhof P (2014): Efficacy of $\beta$ blockers in patients with heart failure plus atrial fibrillation: an individual-patient data meta-analysis. Lancet $\underline{384}$, 2235-2243

Lam L, Lee S-W, Suen CY (1992): Thinning Methodologies-A Comprehensive Survey. IEEE Trans Pattern Anal Mach Intell 14, 869-885

Landstrom AP, Kellen CA, Dixit SS, van Oort RJ, Garbino A, Weisleder N, Ma J, Wehrens XHT, Ackerman MJ (2011): Junctophilin-2 expression silencing causes cardiocyte hypertrophy and abnormal intracellular calcium-handling. Circ Heart Fail 4, 214-223

Landstrom AP, Beavers DL, Wehrens XHT (2014): The junctophilin family of proteins: From bench to bedside. Trends Mol Med 20, 353-362

Lang RE, Thölken H, Ganten D, Luft FC, Ruskoaho H, Unger T (1985): Atrial natriuretic factor--a circulating hormone stimulated by volume loading. Nature 314, 264-266

Larsson HP (2010): How is the heart rate regulated in the sinoatrial node? Another piece to the puzzle. J Gen Physiol 136, 237-241

Lehnart SE, Wehrens XHT, Reiken S, Warrier S, Belevych AE, Harvey RD, Richter W, Jin S-LC, Conti M, Marks AR (2005): Phosphodiesterase 4D deficiency in the ryanodinereceptor complex promotes heart failure and arrhythmias. Cell 123, 25-35

Lohse MJ, Engelhardt S, Eschenhagen T (2003): What is the role of beta-adrenergic signaling in heart failure? Circ Res $\underline{93}$, 896-906

Lüss I, Boknik P, Jones LR, Kirchhefer U, Knapp J, Linck B, Lüss H, Meissner A, Müller FU, Schmitz W (1999): Expression of Cardiac Calcium Regulatory Proteins in Atrium v Ventricle in Different Species. J Mol Cell Cardiol 31, 1299-1314 
Minamisawa S, Oshikawa J, Takeshima H, Hoshijima M, Wang Y, Chien KR, Ishikawa Y, Matsuoka R (2004): Junctophilin type 2 is associated with caveolin-3 and is down-regulated in the hypertrophic and dilated cardiomyopathies. Biochem Biophys Res Commun $\underline{325}$, 852-856

Mitchell JH, Gupta DN, Payne RM (1965): Influence of Atrial Systole on Effective Ventricular Stroke Volume. Circ Res 17, 11-18

Mogensen UM, Jhund PS, Abraham W'T, Desai AS, Dickstein K, Packer M, Rouleau JL, Solomon SD, Swedberg K, Zile MR (2017): Type of Atrial Fibrillation and Outcomes in Patients With Heart Failure and Reduced Ejection Fraction. J Am Coll Cardiol 70, 2490_ 2500

Munro ML, Jayasinghe ID, Wang Q, Quick A, Wang W, Baddeley D, Wehrens XHT, Soeller C (2016): Junctophilin-2 in the nanoscale organisation and functional signalling of ryanodine receptor clusters in cardiomyocytes. J Cell Sci $\underline{129}$, 4388-4398

Ni L, Scott L, Campbell HM, Pan X, Alsina KM, Reynolds J, Philippen LE, Hulsurkar M, Lagor WR, Li N, Wehrens XHT (2019): Atrial-Specific Gene Delivery Using an AdenoAssociated Viral Vector. Circ Res 124, 256-262

Nickel AG, von Hardenberg A, Hohl M, Löffler JR, Kohlhaas M, Becker J, Reil J-C, Kazakov A, Bonnekoh J, Stadelmaier M (2015): Reversal of Mitochondrial Transhydrogenase Causes Oxidative Stress in Heart Failure. Cell Metab 22, 472-484

Nikolaidou T, Cai XJ, Stephenson RS, Yanni J, Lowe T, Atkinson AJ, Jones CB, Sardar R, Corno AF, Dobrzynski H (2015): Congestive Heart Failure Leads to Prolongation of the PR Interval and Atrioventricular Junction Enlargement and Ion Channel Remodelling in the Rabbit. PloS One $\underline{10}$, e0141452

Nisbet AM, Camelliti P, Walker NL, Burton FL, Cobbe SM, Kohl P, Smith GL (2016): Prolongation of atrio-ventricular node conduction in a rabbit model of ischaemic cardiomyopathy: Role of fibrosis and connexin remodelling. J Mol Cell Cardiol 94, 54-64

Noujaim SF, Lucca E, Muñoz V, Persaud D, Berenfeld O, Meijler FL, Jalife J (2004): From mouse to whale: a universal scaling relation for the PR Interval of the electrocardiogram of mammals. Circulation 110, 2802-2808

Parton RG, Way M, Zorzi N, Stang E (1997): Caveolin-3 associates with developing Ttubules during muscle differentiation. J Cell Biol 136, 137-154

Patten RD, Hall-Porter MR (2009): Small animal models of heart failure: development of novel therapies, past and present. Circ Heart Fail 2, 138-144

Pessina F, Gamberucci A, Chen J, Liu B, Vangheluwe P, Gorelli B, Lorenzini S, Spiga O, Trezza A, Sgaragli G, Saponara S (2018): Negative chronotropism, positive inotropism and lusitropism of 3,5-di-t-butyl-4-hydroxyanisole (DTBHA) on rat heart preparations occur through reduction of RyR2 Ca2+ leak. Biochem Pharmacol 155, 434-443 
Prosser BL, Ward CW, Lederer WJ (2010): Subcellular Ca2+ signaling in the heart: the role of ryanodine receptor sensitivity. J Gen Physiol 136, 135-142

Rahimtoola SH, Ehsani A, Sinno MZ, Loeb HS, Rosen KM, Gunnar RM (1975): Left atrial transport function in myocardial infarction: Importance of its booster pump function. Am J Med 모, 686-694

Ramadeen A, Connelly KA, Leong-Poi H, Hu X, Fujii H, Krieken R van, Laurent G, Holub BJ, Bazinet RP, Dorian P (2012): N-3 polyunsaturated fatty acid supplementation does not reduce vulnerability to atrial fibrillation in remodeling atria. Heart Rhythm $\underline{9}, 1115$ 1122.e4

Reiser PJ, Kline WO (1998): Electrophoretic separation and quantitation of cardiac myosin heavy chain isoforms in eight mammalian species. Am J Physiol-Heart Circ Physiol 274, H1048-H1053

Reynolds JO, Chiang DY, Wang W, Beavers DL, Dixit SS, Skapura DG, Landstrom AP, Song L-S, Ackerman MJ, Wehrens XHT (2013): Junctophilin-2 is necessary for T-tubule maturation during mouse heart development. Cardiovasc Res 100, 44-53

Reynolds JO, Quick AP, Wang Q, Beavers DL, Philippen LE, Showell J, Barreto-Torres G, Thuerauf DJ, Doroudgar S, Glembotski CC, Wehrens XHT (2016): Junctophilin-2 gene therapy rescues heart failure by normalizing RyR2-mediated Ca2+ release. Int J Cardiol 225, 371-380

Sanders P, Morton JB, Davidson NC, Spence SJ, Vohra JK, Sparks PB, Kalman JM (2003): Electrical remodeling of the atria in congestive heart failure: electrophysiological and electroanatomic mapping in humans. Circulation $\underline{108}, 1461-1468$

Schoeller R, Andresen D, Büttner P, Oezcelik K, Vey G, Schröder R (1993): First- or second-degree atrioventricular block as a risk factor in idiopathic dilated cardiomyopathy. Am J Cardiol $\underline{71}, 720-726$

Schotten U, Verheule S, Kirchhof P, Goette A (2011): Pathophysiological Mechanisms of Atrial Fibrillation: A Translational Appraisal. Physiol Rev 91, 265-325

Segnani C, Ippolito C, Antonioli L, Pellegrini C, Blandizzi C, Dolfi A, Bernardini N (2015): Histochemical Detection of Collagen Fibers by Sirius Red/Fast Green Is More Sensitive than van Gieson or Sirius Red Alone in Normal and Inflamed Rat Colon. PloS One 10, e0144630

Smyrnias I, Mair W, Harzheim D, Walker SA, Roderick HL, Bootman MD (2010): Comparison of the T-tubule system in adult rat ventricular and atrial myocytes, and its role in excitation-contraction coupling and inotropic stimulation. Cell Calcium $\underline{47}, 210-223$

Soeller C, Cannell MB (1999): Examination of the transverse tubular system in living cardiac rat myocytes by 2-photon microscopy and digital image-processing techniques. Circ Res 84, 266-275 
Song L-S, Sobie EA, McCulle S, Lederer WJ, Balke CW, Cheng H (2006): Orphaned ryanodine receptors in the failing heart. Proc Natl Acad Sci U S A 103, 4305-4310

Statistisches Bundesamt (Destatis) (Hrsg.): Diagnosedaten der Patienten und Patientinnen in Krankenhäuser in Deutschland 2016. Statistisches Bundesamt, Wiesbaden 2017; https://www.destatis.de/DE/Themen/Gesellschaft-

Umwelt/Gesundheit/Krankenhaeuser/Publikationen/Downloads-

Krankenhaeuser/diagnosedaten-krankenhaus-2120621167005.html; Zugriff am 08.05.2019

Statistisches Bundesamt (Destatis) (Hrsg.): Todesursachen in Deutschland 2016. Statistisches Bundesamt, Wiesbaden 2018; https://www.destatis.de/DE/Themen/GesellschaftUmwelt/Gesundheit/Todesursachen/Publikationen/Downloads-

Todesursachen/todesursachenstatistik-5232101167015.html; Zugriff am 08.05.2019

Takeshima H, Komazaki S, Nishi M, Iino M, Kangawa K (2000): Junctophilins: A Novel Family of Junctional Membrane Complex Proteins. Mol Cell $\underline{6}, 11-22$

Talman V, Ruskoaho H (2016): Cardiac fibrosis in myocardial infarction-from repair and remodeling to regeneration. Cell Tissue Res $\underline{365}, 563-581$

Thul R, Coombes S, Roderick HL, Bootman MD (2012): Subcellular calcium dynamics in a whole-cell model of an atrial myocyte. Proc Natl Acad Sci U S A $\underline{109}, 2150-2155$

van Oort RJ, Garbino A, Wang W, Dixit SS, Landstrom AP, Gaur N, De Almeida AC, Skapura DG, Rudy Y, Burns AR (2011): Disrupted junctional membrane complexes and hyperactive ryanodine receptors after acute junctophilin knockdown in mice. Circulation $\underline{123}, 979-988$

Wagner E, Lauterbach MA, Kohl T, Westphal V, Williams GSB, Steinbrecher JH, Streich J-H, Korff B, Tuan H-TM, Hagen B (2012): Stimulated emission depletion live-cell superresolution imaging shows proliferative remodeling of T-tubule membrane structures after myocardial infarction. Circ Res 111, 402-414

Wagner E, Brandenburg S, Kohl T, Lehnart SE (2014): Analysis of Tubular Membrane Networks in Cardiac Myocytes from Atria and Ventricles. JoVE J Vis Exp e51823-e51823

Walden AP, Dibb KM, Trafford AW (2009): Differences in intracellular calcium homeostasis between atrial and ventricular myocytes. J Mol Cell Cardiol 46, $463-473$

Wang TJ, Larson MG, Levy D, Vasan RS, Leip EP, Wolf PA, D’Agostino RB, Murabito JM, Kannel WB, Benjamin EJ (2003): Temporal relations of atrial fibrillation and congestive heart failure and their joint influence on mortality: the Framingham Heart Study. Circulation $\underline{107}, 2920-2925$

Wang Y, Chen B, Huang C-K, Guo A, Wu J, Zhang X, Chen R, Chen C, Kutschke W, Weiss RM (2018): Targeting Calpain for Heart Failure Therapy: Implications From Multiple Murine Models. JACC Basic Transl Sci $\underline{3}, 503-517$ 
Wankerl M, Böhm M, Morano I, Rüegg JC, Eichhorn M, Erdmann E (1990): Calcium sensitivity and myosin light chain pattern of atrial and ventricular skinned cardiac fibers from patients with various kinds of cardiac disease. J Mol Cell Cardiol 22, 1425-1438

Weber KT, Sun Y, Bhattacharya SK, Ahokas RA, Gerling IC (2013): Myofibroblastmediated mechanisms of pathological remodelling of the heart. Nat Rev Cardiol 10, 15-26

Wehrens XHT, Lehnart SE, Reiken SR, Marks AR (2004): Ca2+/calmodulin-dependent protein kinase II phosphorylation regulates the cardiac ryanodine receptor. Circ Res 94, e61-70

Wehrens XHT, Lehnart SE, Marks AR (2005): Intracellular calcium release and cardiac disease. Annu Rev Physiol 67, 69-98

Wehrens XHT, Lehnart SE, Reiken S, Vest JA, Wronska A, Marks AR (2006): Ryanodine receptor/calcium release channel PKA phosphorylation: a critical mediator of heart failure progression. Proc Natl Acad Sci U S A $103,511-518$

Wu H-D, Xu M, Li R-C, Guo L, Lai Y-S, Xu S-M, Li S-F, Lü Q-L, Li L-L, Zhang H-B (2012): Ultrastructural remodelling of Ca2+ signalling apparatus in failing heart cells. Cardiovasc Res $\underline{95}, 430-438$

Wu S, Cheng C-M, Lanz RB, Wang T, Respress JL, Ather S, Chen W, Tsai S-J, Wehrens XHT, Tsai M-J, Tsai SY (2013): Atrial identity is determined by a COUP-TFII regulatory network. Dev Cell 25, 417-426

Xu M, Zhou P, Xu S-M, Liu Y, Feng X, Bai S-H, Bai Y, Hao X-M, Han Q, Zhang Y, Wang S-Q (2007): Intermolecular Failure of L-type Ca2+ Channel and Ryanodine Receptor Signaling in Hypertrophy. PLOS Biol $\underline{5}$, e21

Xu M, Wu H-D, Li R-C, Zhang H-B, Wang M, Tao J, Feng X-H, Guo Y-B, Li S-F, Lai S-T (2012): Mir-24 regulates junctophilin-2 expression in cardiomyocytes. Circ Res $\underline{111}$, 837841

Zhang H-B, Li R-C, Xu M, Xu S-M, Lai Y-S, Wu H-D, Xie X-J, Gao W, Ye H, Zhang Y-Y (2013): Ultrastructural uncoupling between T-tubules and sarcoplasmic reticulum in human heart failure. Cardiovasc Res ㅁ, 269-276 


\section{Danksagung}

Meinen besonderen Dank möchte ich meinem Betreuer Prof. Dr. Stephan E. Lehnart aussprechen, der mich während dieser Arbeit intensiv und professionell begleitet hat. Danke für die Überlassung des Themas, für stetes Interesse an meiner Promotion, für hilfreiche wöchentliche ,Jours fixes“ und Lab-Meetings, für schnelle und hilfreiche Korrekturen im Rahmen der Entstehung dieser Arbeit, aber auch beim Verfassen des Abstracts und Erstellen des Posters, das ich 2017 auf dem jährlichen Kongress der DGK in Mannheim mit Erfolg vorstellen durfte.

Vielen Dank an Prof. Dr. Dörthe M. Katschinski für die Bereitschaft zur Ko-Betreuung dieser Arbeit und für hilfreiche Anmerkungen im Rahmen der Progress Reports. Ganz herzlichen Dank im Übrigen für die hervorragende Lehre im Institut für Physiologie, die mir aus der vorklinischen Studienzeit in besonderer Erinnerung geblieben ist und sicher mitverantwortlich war für das Interesse an Grundlagenforschung.

Sehr persönlich danke ich meinem direkten Betreuer Dr. Sören Brandenburg, der mich zu jedem Zeitpunkt der Arbeit vom ersten Tag der Einarbeitung im Labor im Oktober 2015 bis zum Verfassen und Korrigieren der Dissertation strukturiert, tatkräftig, ruhig, unkompliziert und effizient unterstützt hat. Danke für unzählige Erklärungen, die Einführung in wissenschaftliches Arbeiten, Arbeit im Labor und am Mikroskop, Datenauswertung und Umgang mit ImageJ, für interkontinentale Skypegespräche und nächtliche E-Mails. Ebenso vielen Dank für die Unterstützung bei der Präsentation unserer Daten im Rahmen der Postersession auf dem DGK-Kongress 2017.

Aus der Arbeitsgruppe der Translationalen Kardiologie danke ich weiterhin vor allem Dr. Eva Wagner, die mich während der Zeit meiner Experimente, in der wir uns einen Laborraum teilten, außerordentlich kompetent, geduldig, wohlwollend und warmherzig unterstützt hat. Ich danke Birgit Schumann, Brigitte Korff und Christiane Schulz für ihre exzellente technische, administrative und menschliche Unterstützung.

Allen anderen Kollegen aus der Arbeitsgruppe, nämlich Dr. Gunnar Weninger, Dr. Tobias Kohl, Dr. Miroslav Dura sowie den Doctoribus in spe Jonas Peper und Daniel Kownatzki-Danger danke ich für die freundschaftliche, kollegiale Atmosphäre und die schöne gemeinsame Zeit.

Danke an Roland Blume und Marcel Zoremba für die Durchführung der echokardiographischen Untersuchungen sowie an Beate Knocke für die Auswertung der echokardiographischen Daten. 
Ich danke Prof. Dr. Gerd Hasenfuß, durch dessen Engagement dieses disziplinübergreifende Projekt im Rahmen des Sonderforschungsbereichs SFB 1002 „Modulatorische Einheiten bei Herzinsuffizienz" möglich geworden ist. Danke für das stete Interesse an meiner Arbeit und für die Förderung. Diese Arbeit wurde im SFB 1002 vom Teilprojekt A09 und den Serviceprojekten S01 und S02 unterstützt.

Als Stipendiat der Studienstiftung des Deutschen Volkes und des Deutschen Zentrums für Herzkreislaufforschung bedanke ich mich für die hilfreiche ideelle und finanzielle Förderung. 